\title{
Green-Schwarz automorphisms and 6D SCFTs
}

\author{
Fabio Apruzzi, ${ }^{a, b}$ Jonathan J. Heckman ${ }^{b}$ and Tom Rudelius ${ }^{c}$ \\ ${ }^{a}$ Department of Physics, University of North Carolina, \\ Chapel Hill, NC 27599, U.S.A. \\ ${ }^{b}$ Department of Physics and Astronomy, University of Pennsylvania, \\ Philadelphia, PA 19104, U.S.A. \\ ${ }^{c}$ Jefferson Physical Laboratory, Harvard University, \\ Cambridge, MA 02138, U.S.A. \\ E-mail: fabio.apruzzi@unc.edu, jheckman@sas.upenn.edu, \\ rudelius@g.harvard.edu
}

ABSTRACT: All known interacting 6D superconformal field theories (SCFTs) have a tensor branch which includes anti-chiral two-forms and a corresponding lattice of string charges. Automorphisms of this lattice preserve the Dirac pairing and specify discrete global and gauge symmetries of the $6 \mathrm{D}$ theory. In this paper we compute this automorphism group for $6 \mathrm{D}$ SCFTs. This discrete data determines the geometric structure of the moduli space of vacua. Upon compactification, these automorphisms generate Seiberg-like dualities, as well as additional theories in discrete quotients by the $6 \mathrm{D}$ global symmetries. When a perturbative realization is available, these discrete quotients correspond to including additional orientifold planes in the string construction.

Keywords: Conformal Field Models in String Theory, Discrete Symmetries, F-Theory, Field Theories in Higher Dimensions

ARXIV EPRINT: 1707.06242 


\section{Contents}

1 Introduction $\quad 2$

2 Green-Schwarz automorphisms 5

$2.1 \mathcal{N}=(2,0)$ theories 8

$2.2 \mathcal{N}=(1,0)$ theories $\quad 9$

2.2.1 A-type endpoints 11

2.2.2 D-type endpoints 13

2.3 Higgs branch tuning 14

$\begin{array}{lll}2.4 \text { RG flows } & 14\end{array}$

3 Tensor branch moduli space $\quad 15$

$\begin{array}{lll}3.1 & \text { Tessellating } \mathbb{R}^{T} & 18\end{array}$

3.2 Forbidden zones 20

4 Compactification $\quad 21$

4.1 Descendants of the 6D Weyl group 21

4.1.1 4D theories $\quad 22$

4.1.2 2D theories 23

4.2 Discrete quotients of a 6D SCFT 23

4.2.1 $4 \mathrm{D} \mathcal{N}=2$ SCFTs and generalized quivers $\quad 27$

$\begin{array}{lll}4.2 .2 & \text { A-type } \mathcal{S}_{\Gamma} \text { theories } & 29\end{array}$

4.2.3 D-type $\mathcal{S}_{\Gamma}$ theories $\quad 32$

$\begin{array}{lll}\text { 4.2.4 } & \text { E-type } \mathcal{S}_{\Gamma} \text { theories } & 38\end{array}$

5 Conclusions $\quad 42$

A Anomaly polynomial and Green-Schwarz redundancy 43

A.1 All Green-Schwarz terms via analytic continuation $\quad 45$

$\begin{array}{lll}\text { A.2 Examples } & 47\end{array}$

A.2.1 Rank $Q$ E-string theory $\quad 47$

$\begin{array}{lll}\text { A.2.2 } & \mathrm{SO}(10)-\mathrm{Sp}(1) & 49\end{array}$

A.2.3 $\mathrm{SU}\left(N_{1}\right)-\mathrm{SU}\left(N_{2}\right) \quad 50$

A.2.4 $\mathrm{Sp}(1)-\mathrm{SO}(10)-\mathrm{Sp}(1) \quad 50$ 


\section{Introduction}

Six-dimensional supeconformal field theories (SCFTs) provide a higher-dimensional perspective on many aspects of lower-dimensional quantum field theories. A canonical example of this phenomenon is the compactification of theories with $\mathcal{N}=(2,0)$ supersymmetry to four dimensions. Reduction on a $T^{2}$ yields a geometric perspective on S-duality for $\mathcal{N}=4$ Super Yang-Mills theory, and compactification on a more general Riemann surface leads to $\mathcal{N}=2$ generalizations of S-duality [1-4]. Similar considerations hold for compactifications to lower-dimensional systems. The comparatively large number of $6 \mathrm{D}$ theories with $\mathcal{N}=(1,0)$ supersymmetry recently classified in references [5-7] (see also [8-10]) provides a vast generalization of this paradigm to lower-dimensional physical theories with less supersymmetry. For earlier work on the construction and study of 6D SCFTs, see for example [11-24]. Given this, it is important to isolate calculable quantities for these theories and their compactified lower-dimensional descendants. For a partial list of references on this topic, see e.g. [25-70].

One of the robust "topological" elements of all $(2,0)$ theories is its Dirac pairing for string charges. These pairings are classified by the Dynkin diagrams of the simply laced algebras, a fact which is transparent in the IIB realization of these theories via compactification on $\mathbb{C}^{2} / \Gamma_{\mathrm{ADE}}$, with $\Gamma_{\mathrm{ADE}}$ an ADE discrete subgroup of $\mathrm{SU}(2)$. The resolution of this orbifold singularity yields a geometric realization of the corresponding ADE root system, and upon compactification on a $T^{2}$ yields an $\mathcal{N}=4$ Super Yang-Mills theory with ADE gauge group.

The geometry of the root lattice also dictates the structure of the moduli space. For example, letting $\mathcal{W}$ denote the Weyl group, the $(2,0)$ tensor branch moduli space decomposes into a positive cone $\mathbb{R}^{5 T} / \mathcal{W}$, where $T$ is the number of $\mathcal{N}=(2,0)$ tensor multiplets (see e.g. [71]). This structure persists for lower-dimensional compactifications. For example, the Coulomb branch of $\mathcal{N}=4$ Super Yang-Mills theory with gauge group $G$ is $\mathbb{R}^{6 T} / \mathcal{W}_{G}$. As another example, compactification to two-dimensional theories provides a natural analogue of this in which the Weyl group defines an orbifold CFT, with twisted sectors given by its conjugacy classes (see e.g. [72, 73]).

In this paper we determine the analogous structure for all 6D SCFTs realized via Ftheory compactification. More precisely, we shall be interested in the discrete gauge and global symmetries associated with the lattice of string charges.

The main tool at our disposal is the topological nature of the Green-Schwarz-SagnottiWest terms present in the tensor branch deformation of a 6D SCFT [74-77]. These couplings take the schematic form:

$$
L_{6 D} \supset \int \mu_{i, g} B^{(i)} \wedge \operatorname{Tr}\left(F^{(g)} \wedge F^{(g)}\right),
$$

Here, $B^{(i)}$ is an anti-chiral two-form with $i$ an index labelling the $(1,0)$ tensor multiplets, and $F^{(g)}$ is a two-form field strength with $g$ an index which runs over both dynamical gauge fields as well as background fields. Such background fields are present when we have a non-trivial background flavor symmetry, R-symmetry, or spin connection. Anomaly 
cancellation enforces a rather rigid structure on the coefficients $\mu_{i, g}$. Even so, there is at first some apparent freedom in how we specify their values. Indeed, the invariant quantity which enters the anomaly polynomial eight-form is:

$$
M_{g, h}=\mu_{g, i}^{T}\left(\frac{1}{A}\right)^{i j} \mu_{j, h}
$$

where here, $A_{i j}$ is the Dirac pairing for the $6 \mathrm{D}$ string charges, which is interpreted geometrically as the intersection pairing for the base of an F-theory compactification on an elliptically fibered Calabi-Yau threefold.

This apparent ambiguity would at first seem to suggest more than one set of GreenSchwarz terms will give a consistent 6D SCFT, but it is resolved once we impose the further constraint that all effective strings have positive tension. Geometrically, this is the condition that each effective divisor of the F-theory base has positive volume. Different choices of the $\mu$ 's correspond to formally continuing some tensions to negative values. In field theory terms, we are simply performing a mild version of "duality" in six dimensions. The reason for the terminology is that in lower dimensions, these operations often appear as Seiberg-like dualities, in accord with both the brane moves studied in reference [78] as well as the geometric realization of such maneuvers considered in reference [79].

As an illustrative example, consider the case of $N$ M5-branes probing the geometry $\mathbb{R}_{\perp} \times \mathbb{C}^{2} / \Gamma_{\mathrm{ADE}}$. Moving onto a partial tensor branch corresponds to keeping the M5branes at the $\mathrm{ADE}$ singularity, whilst moving them to separate points on the $\mathbb{R}_{\perp}$ factor. The relative separation between the M5-branes defines a chamber of the partial tensor branch. Moving the M5-branes through one another amounts to formally continuing some vevs to negative values. This leads to a compensating shift in the Green-Schwarz terms, as dictated by the ambiguity in specifying the $\mu$ 's of line (1.2).

Such ambiguities are captured in terms of linear maps on the lattice of string charges $\Lambda$ that preserve the intersection pairing $A_{i j}$. These linear maps are automorphisms of the lattice, or equivalently of the Dirac / intersection pairing. They form a group, which we denote as $\operatorname{Aut}(\Lambda)$. Our goal in this work will be to determine $\operatorname{Aut}(\Lambda)$ for all $6 \mathrm{D}$ SCFTs and to explain how it dictates the structure of lower-dimensional theories obtained from compactification. In some sense this data is complementary to the defect group $\Lambda^{*} / \Lambda$ of a 6D SCFT [44].

To compute $\operatorname{Aut}(\Lambda)$, it is convenient to use the F-theory realization of $6 \mathrm{D}$ SCFTs. The main point is that all of the associated lattices $\Lambda$ are readily available in this context, and are specified by a configuration of $\mathbb{P}^{1}$ 's which can simultaneously contract to zero size. This is the condition that the intersection pairing $A$ is positive definite. F-theory imposes additional conditions on admissible $A$ 's, since we must also be able to define a consistent elliptically fibered Calabi-Yau threefold over a candidate base. In F-theory, we can also blowdown curves of self-intersection -1 in a descending sequence until we are left with a configuration of curves, none of which has self-intersection -1 . The endpoint configuration of curves also defines a lattice $\Lambda_{\text {end }}$. Its automorphism group is related to that of $\Lambda$ as:

$$
\operatorname{Aut}(\Lambda)=\operatorname{Aut}\left(\Lambda_{\text {end }}\right) \times \operatorname{Aut}\left(\mathfrak{s p}_{Q}\right)
$$


where $\operatorname{Aut}\left(\mathfrak{s p}_{Q}\right)$ is the automorphism group for the root lattice of $\mathfrak{s p}_{Q}$, and $Q$ indicates the number of blowdowns of -1 curves which must be performed to pass from $\Lambda$ to $\Lambda_{\text {end }}$. An additional property of $\operatorname{Aut}\left(\Lambda_{\text {end }}\right)$ is the existence of a maximal normal subgroup $\mathcal{W}_{\text {end }}$, which is a close analog of the Weyl group present for $\mathcal{N}=(2,0)$ theories. In terms of this, we find a further refinement:

$$
\operatorname{Aut}\left(\Lambda_{\text {end }}\right)=\mathcal{O}_{\text {end }} \ltimes \mathcal{W}_{\text {end }} .
$$

The physical interpretation of this discrete data is as follows: first, we see that the group $\operatorname{Aut}(\Lambda)$ contains a maximal normal subgroup $\mathcal{W}=\mathcal{W}_{\text {end }} \times \operatorname{Aut}\left(\mathfrak{s p}_{Q}\right)$, which we identify with discrete gauge symmetries of the system. Additionally, $\mathcal{O}_{\text {end }}$ is a candidate global discrete symmetry. This is borne out by the fact that the base of the F-theory model enjoys a group of discrete isometries specified by $\mathcal{O}_{\text {end }}$. This is true both at the conformal fixed point as well as in the resolved geometry. In the full F-theory model, this symmetry may be broken because there could be a non-trivial elliptic fibration. Said differently, this global symmetry depends on the Higgs branch moduli.

The structure of the tensor branch moduli space for $\mathcal{N}=(1,0)$ theories can be far more involved than it is for $\mathcal{N}=(2,0)$ theories. For example, though there is still a notion of a fundamental domain of moduli space for a theory with $T$ tensor multiplets, the orbits of this patch under the automorphism group sometimes do not produce a tessellation of $\mathbb{R}^{T}$, leading to non-trivial forbidden zones. These different possibilities are conveniently handled using the F-theory characterization of 6D SCFTs, where these moduli correspond to resolution parameters of curves.

Though we leave a more complete analysis for future work, we can already point to the ways in which the data of $\operatorname{Aut}(\Lambda)$ shows up in compactifications of $6 \mathrm{D}$ SCFTs. First of all, the discrete gauge symmetries $\mathcal{W}$ lead to Seiberg-like dualities once we compactify and flow to another four-dimensional theory. One particularly interesting feature of 6D SCFTs is the presence on the tensor branch of generalizations of quiver gauge theories with exceptional gauge groups and "conformal matter." We also consider the related structures for $2 \mathrm{~d}$ theories obtained from compactifying on a four-manifold.

The discrete global symmetries of the $6 \mathrm{D}$ theory also lead to several novel structures upon further compactification. For example, adding a chemical potential for the background global symmetries of $\mathcal{O}_{\text {end }}$ yields a new theory which is the equivalent of introducing "discrete twists" in compactifications of class $\mathcal{S}$ theories (see e.g. [80-82]). Another (and conceptually distinct) operation of a more stringy flavor is associated with formally quotienting the theory by this symmetry. An interesting feature of this is that it also provides a field theoretic characterization of various orientifold planes in compactifications of F-theory, including the effects of $O 7^{+}$-planes (see e.g. [83-85]), along the lines proposed in reference [86].

The rest of this paper is organized as follows: first, in section 2 we discuss in general terms the automorphism group for a lattice of strings, and the physical data it captures in compactifications of a 6D SCFT, and compute it for all 6D SCFTs. Section 3 studies the structure of the tensor branch moduli space, as dictated by the automorphism group. Section 4 presents some examples of how the automorphism group specifies defining data 
in compactifications of 6D SCFTs, including the realization of various orientifold planes. We present our conclusions and directions for future research in section 5. Some additional details on how to calculate the anomaly polynomial of a 6D SCFT via "analytic continuation" in the rank of the gauge groups present on the tensor branch are presented in appendix A.

\section{Green-Schwarz automorphisms}

One of the essential elements in all known interacting 6D SCFTs is the existence of a tensor branch. On this branch, we have a collection of $\mathcal{N}=(1,0)$ tensor multiplets. Recall that a tensor multiplet contains a real scalar $t$, an anti-chiral two-form potential $B_{\mu \nu}^{(-)}$with antiself-dual field strength, and corresponding fermionic superpartners. The appearance of a two-form potential signals the presence of strings, with tension controlled by the vevs of the real scalars. The conformal fixed point corresponds to taking all $t$ 's to zero simultaneously. In a theory with $T$ tensor multiplets, the Dirac pairing for string charges is a $T \times T$ positive definite symmetric matrix which acts on the lattice of string charges via a canonical pairing:

$$
A: \Lambda \rightarrow \Lambda \text {. }
$$

This pairing also specifies the metric on moduli space for the $t$ 's. Indeed, indexing the multiplets by the variables $i$ and $j$, the metric on moduli space takes the form $A_{i j} d t^{i} d t^{j}$, in the obvious notation. In this coordinate system, the tension of a string is given by:

$$
t_{i}=A_{i j} t^{j}
$$

An important quantity in all 6D SCFTs is the associated anomaly polynomial. This is a formal eight-form constructed from the background field strengths for the $\mathrm{SU}(2) \mathrm{R}$ symmetry, the curvature of the spin connection, and possible flavor symmetries. In addition to the one loop contribution from chiral modes, there are additional "tree level" contributions from the Green-Schwarz terms:

$$
L_{6 D} \supset \int \mu_{i, g} B^{(i)} \wedge \operatorname{Tr}\left(F^{(g)} \wedge F^{(g)}\right),
$$

where here we also include possible couplings to vector multiplets and their associated gauge field strengths. The contribution to the eight-form anomaly polynomial takes the form:

$$
I_{8 D} \supset \mu_{g, i}^{T}\left(\frac{1}{A}\right)^{i j} \mu_{j, h} \operatorname{Tr}\left(F^{(g)}\right)^{2} \operatorname{Tr}\left(F^{(h)}\right)^{2} .
$$

The anomaly polynomial determines, for example, the conformal anomalies of the 6D SCFT (see e.g. [52, 87]). Another important feature of the anomaly polynomial is that when the number of simple gauge group factors and tensor multiplets is equal, the $\mu$ 's are square matrices and there is then a unique solution to the anomaly cancellation conditions [41, 52] (for additional explicit calculations see also [88-90]). This can also be extended to all 6D SCFTs by interpreting "unpaired tensors" as a generalized type of 6D conformal matter [6]. 
In appendix A we present another method in which we formally introduce a possibly trivial gauge group to pair with each such tensor multiplet.

The only quantity which actually enters into the anomaly polynomial is the combination:

$$
M_{g, h}=\mu_{g, i}^{T}\left(\frac{1}{A}\right)^{i j} \mu_{j, h}
$$

Based on this, it is natural to ask whether there is more than one choice of $\mu$ 's available. The main point is that once we demand all string tensions are positive, namely $t_{i}>0$, we have well defined couplings to the chemical potentials defined by the anti-chiral two-forms. As such, there is a unique choice for the $\mu_{i, g}$ in this patch of moduli space. Provided it makes sense, we can ask what happens to the spectrum of strings if we now pass to formally negative values of some of the $t_{i}$ 's. We clearly must seek a new basis of positive tension objects, and correspondingly the values of the $\mu_{i, g}$ may change.

To determine the geometry of the tensor branch moduli space, we seek integral linear transformations $\sigma_{i}^{j}$ of the coefficients $\mu_{i, g}$ which act on the tensor index:

$$
\mu_{i, g} \mapsto \sigma_{i}^{j} \mu_{j, g}
$$

and preserve the form of the anomaly polynomial, i.e. they preserve the matrix $M_{g, h}$. Equivalently, we seek all transformations $\sigma$ such that:

$$
\sigma^{T} \cdot \frac{1}{A} \cdot \sigma=\frac{1}{A}
$$

The collection of all such $\sigma$ 's forms a group. First of all, we have the identity element. Second, if we have two transformations $\sigma$ and $\sigma^{\prime}$ which both preserve $M_{g, h}$, then their composition will also preserve $M_{g, h}$. To establish the existence of an inverse element, we first verify that $\sigma^{-1}$ is an integral transformation. To see this, we first compute the determinant of equation (2.7). This yields the relation:

$$
(\operatorname{det} \sigma)^{2}=1
$$

so $\operatorname{det} \sigma= \pm 1$.

As such, the inverse of the integral linear map $\sigma$ will also have integer entries, and so $\sigma^{-1}$ is also an integral transformation. Next, consider the inverse of equation (2.7),

$$
\sigma^{-1} \cdot A \cdot\left(\sigma^{-1}\right)^{T}=A .
$$

Multiplication by $\sigma$ on the left and $\sigma^{T}$ on the right yields

$$
A=\sigma \cdot A \cdot \sigma^{T}
$$

Taking the inverse,

$$
\frac{1}{A}=\left(\sigma^{-1}\right)^{T} \cdot \frac{1}{A} \cdot \sigma^{-1},
$$

so the inverse is also an integral transformation preserving $M_{g, h}$. 
In fact, what we have just established is that the group of $\sigma$ 's is also the automorphism group for the quadratic form defined by $A_{i j}$, the intersection pairing of the lattice $\Lambda$. This is also known as the automorphism group of the lattice, and so we shall often write $\operatorname{Aut}(\Lambda)$ to reflect this fact.

What then is the physical interpretation of this group action? In the case of the $\operatorname{ADE}(2,0)$ theories, there is a further decomposition we can perform:

$$
\operatorname{Aut}(\Lambda)=\mathcal{O}_{\mathrm{ADE}} \ltimes \mathcal{W}_{\mathrm{ADE}},
$$

where $\mathcal{W}_{\mathrm{ADE}}$ is the Weyl group i.e. the group of inner automorphisms of the ADE algebra, and $\mathcal{O}_{\mathrm{ADE}}$ is the group of outer automorphisms. This has a clean interpretation upon compactification on torii.

The $(2,0)$ theories yield maximally supersymmetric gauge theories with ADE gauge algebra. In this case, we can identify $\mathcal{W}_{\text {ADE }}$ with a collection of discrete gauge transformations, and $\mathcal{O}_{\mathrm{ADE}}$ as possible ways to twist the theory to reach non-simply laced algebras in lower dimensions.

In the more general case of $(1,0)$ theories, we do not have the luxury of a lowerdimensional gauge theory when we compactify on torii. Instead, we must make do with the structure already apparent in six dimensions. Along these lines, we see that on the tensor branch, the discrete gauge transformations must flip the sign of at least one tensor branch scalar $t_{i}$. These are the natural analogs of the Weyl group transformations in the $(2,0)$ theories. Indeed, they correspond to redundancies in our description of the tensor multiplets, so we interpret these as discrete gauge symmetries. ${ }^{1}$ Other transformations which leave all moduli positive are the natural analog of the outer automorphisms, and correspond to global symmetries.

From the perspective of F-theory compactification, it is immediate that the data of the global symmetries is indeed intrinsic to a given SCFT. The reason is that we specify a base as a resolution of an orbifold of the form $\mathbb{C}^{2} / \Gamma_{\mathrm{U}(2)}$ for $\Gamma$ a discrete subgroup of $\mathrm{U}(2)$. The discrete isometries of this geometry are the global symmetries. Indeed, even after resolving the singularity, these isometries persist, and in the $(2,0)$ case are what we identify with the outer automorphisms of the corresponding Lie algebra.

More precisely, such isometries of the base are really just candidate global symmetries. Indeed, in a full F-theory compactification we often must specify a non-trivial elliptic fibration to the model. Geometrically, a discrete isometry of the base need not extend to the full Calabi-Yau threefold. In physical terms, the elliptic fibration is controlled by the Higgs branch moduli. So, we see that candidate global symmetries will depend on this data.

Having argued that we can have a discrete global symmetry, it is natural to ask whether we can gauge it, or whether it is actually anomalous (unless supplemented by additional degrees of freedom). This sort of gauging operation does not affect the local structure of

\footnotetext{
${ }^{1}$ One might ask whether these are examples of higher-form discrete symmetries in the sense of reference [91]. One way to see that they are "standard" discrete symmetries rather than higher-form symmetries is that on a topologically trivial background spacetime, such symmetries are abelian, whereas our symmetry group is often non-abelian.
} 
correlation functions, and instead leads to global distinctions in the spectrum of extended objects in the theory. It would be interesting to evaluate the corresponding 't Hooft anomalies, but this is beyond the scope of the present paper.

A related though different operation involves quotienting by such a symmetry, as one would do in an orientifold construction. In such cases, we expect a $(2,0)$ theory to become a $(1,0)$ theory. To illustrate this point, consider the A-type $(2,0)$ theories. These theories possess a $\mathbb{Z}_{2}$ outer automorphism which acts by reflection on the nodes of the Dynkin diagram. In string theory terms, if we attempt to quotient by this symmetry, we need to introduce an orientifold plane. This automatically breaks half of the supersymmetry, yielding a $(1,0)$ theory instead. Additional branes must also be included to locally satisfy Gauss' law constraints. This is consistent with the fact that there is no way to perform a "discrete quotient" of a $(2,0)$ theory which is also $\mathcal{N}=(2,0)$ supersymmetric. Instead, such a quotient yields a $(1,0)$ theory. We study this question in much greater detail in section 4 where we consider discrete quotients of compactified theories.

Our plan in the remainder of this section will be to compute Aut $(\Lambda)$ for all $6 \mathrm{D}$ SCFTs. As a warmup, we first briefly review the case of the $(2,0)$ theories, where this data is captured by the automorphisms of the corresponding ADE root lattice. In the case of the $(1,0)$ SCFTs, there are analogous results, including a generalization of a Weyl group as well as outer automorphisms. There are, however, some important differences in the case of non-generic Higgs branch moduli, a point we return to in section 2.3.

\section{$2.1 \mathcal{N}=(2,0)$ theories}

In this section we consider the automorphism group for the $(2,0)$ theories. In this case, the Green-Schwarz terms of equation (2.3) involve the $\mathfrak{s p}(4)$ R-symmetry background field strength and curvature from the spin connection. Using the classification of $(2,0)$ theories via discrete subgroups $\Gamma_{\mathrm{ADE}} \subset \mathrm{SU}(2)$ and the corresponding IIB backgrounds on $\mathbb{R}^{5,1} \times \mathbb{C}^{2} / \Gamma_{\mathrm{ADE}}$, we also know that the $(1,0)$ tensor branch is geometrically realized as the resolution of these orbifold singularities. Indeed, in the resolved geometry, we have a collection of $\mathbb{P}^{1}$ 's which intersect according to the ADE Dynkin diagram. The automorphism group for each intersection form is simply the automorphism group of the ADE root lattice. All of the automorphism groups take the form of a semi-direct product of the outer automorphisms of the algebra with the inner automorphisms associated with the Weyl group:

$$
\operatorname{Aut}\left(\Lambda_{\mathrm{ADE}}\right)=\mathcal{O}_{\mathrm{ADE}} \ltimes \mathcal{W}_{\mathrm{ADE}} .
$$

In particular, the outer automorphisms for each of the ADE root systems are, for $N>1$ and $M>4$ :

\begin{tabular}{|c|c|c|c|c|c|c|c|}
\hline & $A_{1}$ & $A_{N}$ & $D_{4}$ & $D_{M}$ & $E_{6}$ & $E_{7}$ & $E_{8}$ \\
\hline $\mathcal{O}_{\mathrm{ADE}}$ & 1 & $\mathbb{Z}_{2}$ & $S_{3}$ & $\mathbb{Z}_{2}$ & $\mathbb{Z}_{2}$ & 1 & 1 \\
\hline
\end{tabular}

where $S_{3}$ is the symmetric group on three letters.

The moduli space of the tensor branch is given by $\mathbb{R}^{5 T} / \mathcal{W}_{\mathrm{ADE}}$, where the factor of five is due to the fact that we are dealing with $(2,0)$ rather than $(1,0)$ tensor multiplets. Additionally, compactification on a manifold can be accompanied by a twist by an outer 
automorphism $\mathcal{O}_{\mathrm{ADE}}$, possibly composed with an element of $\mathcal{W}_{\mathrm{ADE}}$. Let us note that this operation can be understood field theoretically as activating a background chemical potential for the discrete flavor symmetry. This is a distinct notion from the operation of "discrete quotient" which we shall encounter in section 4.

\section{$2.2 \mathcal{N}=(1,0)$ theories}

In this subsection we compute the Green-Schwarz automorphisms of all 6D SCFTs. At this point, it is convenient to use the geometric language of F-theory compactification, though we stress that all of this analysis can be carried out in purely field theoretic terms.

In the F-theory realization of $6 \mathrm{D}$ SCFTs, we introduce a non-compact Kähler surface $B$ with some configuration of simultaneously collapsing $\mathbb{P}^{1}$ 's. We obtain a consistent F-theory background when we can also define an elliptically fibered Calabi-Yau threefold with base $\mathcal{B}$. The homology lattice of the base determines the lattice of string charges:

$$
H_{2}^{\mathrm{cpct}}(\mathcal{B}, \mathbb{Z})=\Lambda,
$$

and the intersection pairing corresponds to the Dirac pairing. References [5, 7] provide a classification of all such bases, as well as all possible elliptic fibrations over a given base. For our present purposes, the main point is that each such base $\mathcal{B}$ is generated by starting with an "endpoint configuration" of curves which contains no -1 curves, and then performing some prescribed number of blowups. There is a minimal number of blowups (which may be zero) necessary to define a consistent elliptic fibration, but additional blowups are sometimes possible.

Although the specific geometry depends on the particular location of each such blowup, the structure of the lattice of string charges is insensitive to this data [44, 92]. Indeed, given the endpoint configuration $\mathcal{B}_{\text {end }}$ with lattice $H_{2}^{\text {cpct }}\left(\mathcal{B}_{\text {end }}, \mathbb{Z}\right)=\Lambda_{\text {end }}$, blowing up $Q$ times yields the lattice:

$$
\Lambda=\Lambda_{\text {end }} \oplus \mathbb{Z}^{\oplus Q},
$$

This follows from the fact that for a curve $\Sigma$, blowing up the curve generates a shift in the divisor class as:

$$
[\Sigma] \mapsto[\Sigma]-E
$$

with $E$ the exceptional divisor class. From this perspective, the automorphism group splits into two pieces: the contribution from $\Lambda_{\text {end }}$ and the contribution from $\mathbb{Z}^{\oplus Q}$. In most cases, there is an upper bound on the value of $Q$ dictated by the choice of endpoint configuration. For example, some configurations cannot be blown up at all, the $E_{8}$ lattice being one such case [5].

One important consideration is that this linear change of basis for the lattice can change the intersection pairing. Along these lines, consider two lattices $\Lambda$ and $\Lambda^{\prime}$ which are related to each other by a change of basis, as indicated for example by line (2.17):

$$
L: \Lambda^{\prime} \rightarrow \Lambda .
$$

The intersection pairing of the two lattices are related as

$$
L^{T} \cdot A \cdot L=A^{\prime} .
$$


Automorphisms of the two lattices are related via the transformation

$$
L^{-1} \cdot \sigma \cdot L=\sigma^{\prime}
$$

in the obvious notation. As an example, take $\Lambda$ to be the configuration of two -1 curves which do not intersect, and $\Lambda^{\prime}$ to be the 1,2 configuration. The lattice transformation $L$ is

$$
L=\left[\begin{array}{cc}
1 & -1 \\
0 & 1
\end{array}\right] \text {. }
$$

Taking into account this structure, we see that the automorphism group is not sensitive to the locations of the various blowups. Consequently, we learn that for any choice of blowups of an endpoint configuration, the group $\operatorname{Aut}(\Lambda)$ is given by the product:

$$
\operatorname{Aut}(\Lambda)=\operatorname{Aut}\left(\Lambda_{\text {end }}\right) \times \operatorname{Aut}\left(\mathbb{Z}^{\oplus Q}\right) .
$$

For the factor coming from the rank $Q$ E-string theory, we find that the automorphism group is identical to that for the root system of the Lie algebra $\mathfrak{s p}(Q)$, where in our notation $\mathfrak{s p}(1) \simeq \mathfrak{s u}(2)$. Said differently, this is just the Weyl group of the root system.

Let us discuss each of these factors in turn. In the case of $\operatorname{Aut}\left(\mathbb{Z}^{\oplus Q}\right)$, the group is given by possible flops of each individual curve, as well as permutations amongst these curves. All told, the automorphism group for this part is:

$$
\operatorname{Aut}\left(\mathbb{Z}^{\oplus Q}\right)=S_{Q} \ltimes\left(\mathbb{Z}_{2}\right)^{Q} .
$$

As already remarked, this is also the Weyl group for the Lie algebra $\mathfrak{s p}(Q)$. Indeed, if we consider a particular sequence of blowups, we reach the configuration of curves:

$$
\underbrace{1,2, \ldots, 2}_{Q}
$$

Here and in what follows, the notation $a, b$ denotes a pair of curves of self-intersection $-a$ and $-b$ that intersect at one point. We note that this is also the configuration of curves used to realize the rank $Q$ E-string theory. Upon compactification on a circle, it is well known that the rank $Q$ E-string theory reduces to an $\mathfrak{s p}(Q)$ 5D gauge theory with seven flavors. At the conformal fixed point, this enhances to an $E_{8}$ flavor symmetry.

Consider next the contribution from the factor $\operatorname{Aut}\left(\Lambda_{\text {end }}\right)$. In this case, it is convenient to make use of the explicit classification of endpoint configurations given in reference [5]. These take the form of generalized A- and D-type Dynkin diagrams, while the E-series still involves only the standard -2 curves:

$$
\begin{aligned}
& \text { A-type: } \quad n_{1}, \ldots, n_{l} \\
& \text { D-type: } 2, m_{1}^{2} \ldots, n_{l} \\
& E_{6}: \quad 2,2, \stackrel{2}{2}, 2,2 \\
& E_{7}: \quad 2,2,2,2,2,2 \\
& E_{8}: \quad 2,2, \stackrel{2}{2}, 2,2,2,2 \text {. }
\end{aligned}
$$


In the last three cases, we simply have the automorphism group of the corresponding $E$-type root system. We therefore confine our attention to the A- and D-type endpoint configurations. Most of these computations are a straightforward application of symmetries manifest in the configuration, and we have verified this structure using the software package MAGMA.

\subsubsection{A-type endpoints}

From reference [5], we know that all the A-type end points can be described by a collection of curves of the following form:

$$
M^{1} N^{1} \ldots M^{a} N^{a} \ldots M^{a_{l}} N^{a_{l}},
$$

where $M^{a}=\{22 \ldots 2\}$ is a sequence of $m_{a}$ curves of self-intersection -2 , and $N^{a}=$ $\left\{n_{1} n_{2} \ldots n_{N^{a}}\right\}$ is a sequence of curves with $n_{i}>2$ for all $i$. Here, the notation indicates that we have a collection of curves of self-intersection $-n$, which intersect pairwise at a single point, as indicated by the ordering of the sequence. In fact, from the classification results of [5], we know that $l \leq 3$.

To determine the structure of the automorphism group, and in particular its action on the tensor branch moduli space, we observe that the automorphism group will be a subgroup of that for the A-type configuration with just -2 curves. In this sense, all we really need to do is track the automorphisms which survive when we change from a configuration of -2 curves to one where some curves in the endpoint are replaced by $-n$ curves with $n>2$.

Along these lines, we find that all of the Weyl reflections for the -2 curves naturally survive. Additionally, for a -2 curve with class $\alpha$ which intersects a $-n$ curve with class $\beta$, the change under Weyl reflection is again:

$$
\alpha \mapsto-\alpha \text { and } \beta \mapsto \beta+\alpha \text {. }
$$

What then becomes of the remaining Weyl reflections which act on the $-n$ curves? The basic point is that we are restricted to a very limited class of reflections in which all curves simultaneously transform.

Now, by inspection the automorphism group always contains the element $-I d$. This element is an order two element which acts on the divisor classes as:

$$
\alpha_{i} \mapsto-\alpha_{i}
$$

The symmetries of the endpoint configuration dictate whether this is an element of the normal subgroup of $\operatorname{Aut}(\Lambda)$ which generates discrete gauge symmetries. To see why, we observe that in the case where the configuration of curves enjoys a reflection symmetry, this element is a composition of an outer automorphism and the discrete gauge transformation:

$$
\alpha_{i} \mapsto-\alpha_{T+1-i}
$$

which acts on all of the divisor classes. When there is no such reflection symmetry, the map $-I d$ is already a discrete gauge transformation. Note that for both $-I d$ as well as 
the map of line (2.33), the map is an order two element. Regardless of whether we have a symmetric or asymmetric endpoint configuration of curves, the analog of the Weyl group for an A-type endpoint configuration is then given by:

$$
\mathbb{Z}_{2} \ltimes\left(S_{m_{1}+1} \times \ldots S_{m_{l}+1}\right)
$$

where in the case of an asymmetric endpoint configuration, this is actually a direct product, and in the case of a symmetric endpoint configuration, the $\mathbb{Z}_{2}$ group action is dictated by the map of line (2.33). Note that in the latter case, $\operatorname{Aut}(\Lambda)$ is a semi-direct product involving this $\mathbb{Z}_{2}$ reflection symmetry.

It is also helpful to explicitly spell out the various types of groups we encounter for A-type lattices. The automorphism group of the endpoint configuration divides into four cases:

- When the endpoint is a single curve,

$$
\operatorname{Aut}\left(\Lambda_{\text {end }}\right)=\mathbb{Z}_{2} \text {. }
$$

- When the endpoint is a sequence of $m$ curves of self-intersection -2 , we have

$$
\operatorname{Aut}\left(\Lambda_{\text {end }}\right)=\mathbb{Z}_{2} \ltimes S_{m+1},
$$

where $S_{m+1}$ is the symmetric group on $m+1$ letters.

- When the endpoint configuration is symmetric but contains at least one curve of self-intersection $-x$ for $x \geq 3$, we have:

$$
\operatorname{Aut}\left(\Lambda_{\text {end }}\right)=\mathbb{Z}_{2} \ltimes\left(\mathbb{Z}_{2} \ltimes\left(S_{m_{1}+1} \times \ldots S_{m_{l}+1}\right)\right) .
$$

Here, the product over symmetric groups just involves the Weyl groups of each block of -2 curves. The middle $\mathbb{Z}_{2}$ factor acts as in line (2.33). Note that in contrast to the case of configurations of all -2 curves, the number of automorphisms is drastically smaller. This is a consequence of the fact that the notion of "Weyl reflection" is far more restrictive for curves which do not have self-intersection -2 . Finally, the overall semi-direct product by the leftmost $\mathbb{Z}_{2}$ factor acts on the configuration of curves by left/right reflection.

- In the case where the endpoint configuration does not possess such a $\mathbb{Z}_{2}$ reflection symmetry, we obtain a quite similar answer for the automorphism group:

$$
\operatorname{Aut}\left(\Lambda_{\text {end }}\right)=\mathbb{Z}_{2} \times\left(S_{m_{1}+1} \times \ldots S_{m_{l}+1}\right) .
$$

Let us note that in all cases, the automorphisms of the endpoint configuration takes the general form

$$
\operatorname{Aut}\left(\Lambda_{\text {end }}\right)=\mathcal{O}_{\text {end }} \ltimes \mathcal{W}_{\text {end }},
$$

where $\mathcal{O}_{\text {end }}$ are possible automorphisms in the diagram describing the endpoint configuration of curves, and $\mathcal{W}_{\text {end }}$ is a normal subgroup of $\operatorname{Aut}\left(\Lambda_{\text {end }}\right)$ naturally generalizing the Weyl group. To illustrate the above notions, let us now turn to some explicit examples. 
Examples. As a first example, consider the endpoint configuration of curves:

7232222.

There is no left/right symmetry, and we have $m_{1}=1, m_{2}=4$, so we get

$$
\operatorname{Aut}\left(\Lambda_{\text {end }}\right)=\mathbb{Z}_{2} \times S_{2} \times S_{5} .
$$

Next, consider the endpoint configuration:

$$
322223,
$$

which enjoys a $\mathbb{Z}_{2}$ reflection symmetry. Here, we have $m_{1}=4$ so we get

$$
\operatorname{Aut}\left(\Lambda_{\text {end }}\right)=\mathbb{Z}_{2} \ltimes\left(\mathbb{Z}_{2} \ltimes S_{5}\right) .
$$

Finally, consider the endpoint configuration:

$$
2232322 \text {. }
$$

for which we have

$$
\operatorname{Aut}\left(\Lambda_{\text {end }}\right)=\mathbb{Z}_{2} \ltimes\left(\mathbb{Z}_{2} \ltimes S_{3} \times S_{2} \times S_{3}\right),
$$

and the $\mathbb{Z}_{2}$ associated with reflection exchanges the two $S_{3}$ factors.

\subsubsection{D-type endpoints}

Consider next the D-type endpoint configurations. Much as in the case of the generalized A-type configurations, all of the automorphisms are inherited from the automorphisms of the D-type configuration with just -2 curves.

Recall that in the case of the $D_{N}$ Dynkin diagram with -2 curves, the automorphism group is:

$$
\operatorname{Aut}\left(D_{N}\right)=\mathcal{O}_{D_{N}} \ltimes \mathcal{W}_{D_{N}}
$$

where for $N \geq 5$, the outer automorphism group is $\mathbb{Z}_{2}$, and for $N=4$ it is $S_{3}$. The Weyl group automorphisms are given by:

$$
\mathcal{W}_{D_{N}}=S_{N} \ltimes\left(\mathbb{Z}_{2}\right)^{N} / \mathbb{Z}_{2} .
$$

here, the overall quotient by $\mathbb{Z}_{2}$ is from the kernel of the map $\left(\mathbb{Z}_{2}\right)^{N} \rightarrow \mathbb{Z}_{2}$ given by multiplication of all factors.

Proceeding now to the more general endpoint configurations, we calculate the automorphism group by recognizing that all automorphisms are given by appropriate subgroups of the $\operatorname{Aut}\left(D_{N}\right)$ series. The key point is that the diagram breaks up into pieces partitioned by the $-n$ curve(s) with $n>2$. The only reflection on such curves is given by the long element of the $D_{N}$ Weyl group, and it acts via a $\mathbb{Z}_{2}$ group action. Additionally, we see that the rest of the diagram now breaks up into at most one D-type diagram for -2 curves, and an A-type Dynkin Diagram. Generically, these discrete gauge symmetries decompose as

$$
\mathcal{W}_{D-\text { type }}=\mathbb{Z}_{2} \ltimes\left(\mathcal{W}_{D_{N}} \times \mathcal{W}_{A_{M}}\right)
$$


where $N$ and $M$ denote the number of -2 curves present in the configuration. For a smaller number of curves, additional possibilities are present. For example, we can consider the $D_{4}$ Dynkin diagram as well as the endpoint where the central curve is a -3 curve instead.

Again, we observe that in all cases, the automorphisms of the endpoint configuration take the general form:

$$
\operatorname{Aut}\left(\Lambda_{\text {end }}\right)=\mathcal{O}_{\text {end }} \ltimes \mathcal{W}_{\text {end }},
$$

where $\mathcal{O}_{\text {end }}$ are possible automorphisms in the diagram describing the endpoint configuration of curves, and $\mathcal{W}_{\text {end }}$ is a normal subgroup of $\operatorname{Aut}\left(\Lambda_{\text {end }}\right)$ which is a natural generalization of the Weyl group.

\subsection{Higgs branch tuning}

Our discussion so far has focused on the structure of the tensor branch moduli space and the automorphism group for the lattice of string charges. In the context of physical applications, it is important to understand the interplay between the tensor branch and Higgs branch moduli. In geometric terms, these correspond to Kähler moduli and complex structure moduli, respectively. More precisely, the complex structure are joined by the intermediate Jacobian of the Calabi-Yau in determining the structure of the Higgs branch moduli space.

Additional complex structure moduli can appear through suitable tuning of coefficients in the Weierstrass model. For example, in the case of a configuration of -2 curves realizing an A-type Dynkin diagram, we can consider various fiber enhancements, leading to a rich structure of possible $6 \mathrm{D}$ SCFTs [7]:

$$
\left[\mathfrak{s u}_{k_{0}}\right] \stackrel{\mathfrak{s u}_{k_{1}}}{2}, \stackrel{\mathfrak{s u}_{k_{2}}}{2}, \ldots, \quad{\stackrel{\mathfrak{s u}}{k_{T-1}}}^{2}, \mathfrak{s u}_{k_{T}}\left[\mathfrak{s u}_{k_{T+1}}\right]
$$

where we have indicated the Lie algebra over each -2 curve, as dictated by the singular elliptic fibrations. To the left and the right, we have also indicated non-compact flavor branes. Anomaly cancellation requires $2 k_{i}=k_{i-1}+k_{i+1}$ for all of the gauge groups supported on compact -2 curves.

Now, depending on the nature of our fiber enhancements, we see that the $\mathbb{Z}_{2}$ automorphism corresponding to left/right reflection on the configuration of -2 curves may no longer be a symmetry of the geometry. Indeed, in the above example we would also need to require $k_{i}=k_{T+1-i}$ for such a reflection symmetry to hold. We take this to mean that some of the candidate automorphisms originating from the lattice of string charges may be broken by Higgs branch moduli.

\subsection{RG flows}

It is also natural to study the behavior of the automorphism group under RG flows from one conformal fixed point to another. In a 6D SCFT, supersymmetry preserving flows are limited to deformations triggered by background operator vevs [57] (see also [88, 93]). Under a tensor branch flow, we decompactify some of the curves of the base. Doing so, we see that we pass to a sublattice:

$$
\Lambda_{\mathrm{IR}} \subset \Lambda_{\mathrm{UV}}
$$


Even so, we cannot quite say that the automorphisms of one are always contained in the other. Indeed, we can already see there could be emergent discrete gauge symmetries in the infrared. To illustrate, consider the case of the $6 \mathrm{D}$ SCFT with endpoint 3,3 . After performing one blowup, we reach a consistent F-theory base, namely 4,1,4. The automorphism group of this configuration is:

$$
\operatorname{Aut}\left(\Lambda_{4,1,4}\right)=\left(\mathbb{Z}_{2} \ltimes \mathbb{Z}_{2}\right) \times \mathbb{Z}_{2} .
$$

We can also study the automorphism group obtained from decompactifying one of our curves. Due to the symmetry of the configuration, it is enough to consider the decompactification of either a -4 curve, or a -1 curve. In these two cases, we reach the automorphism groups:

$$
\begin{aligned}
\operatorname{Aut}\left(\Lambda_{4,1}\right) & =\mathbb{Z}_{2} \times \mathbb{Z}_{2} \\
\operatorname{Aut}\left(\Lambda_{4 \oplus 4}\right) & =\mathbb{Z}_{2} \ltimes\left(\mathbb{Z}_{2} \times \mathbb{Z}_{2}\right) .
\end{aligned}
$$

Whereas the group structure of the first case is directly inherited from that of the original UV theory, the case of two disconnected -4 curves is not of this type. For example, when we have two disconnected -4 curves, we can independently reflect these two curves. This is not possible in the original 4,1,4 theory.

More generally, we see that when we decompactify a curve of an endpoint configuration, there is a strict containment relation for the automorphism groups of the endpoints:

$$
\operatorname{Aut}\left(\Lambda_{\mathrm{IR}}^{\mathrm{end}}\right) \subset \operatorname{Aut}\left(\Lambda_{\mathrm{UV}}^{\mathrm{end}}\right)
$$

If, however, we decompactify a curve which is only present after blowing up an endpoint, then we must entertain the possibility of emergent discrete gauge symmetries in the infrared.

Consider next the case of Higgs branch flows. In these cases, we see that if we start at a tuned point on the Higgs branch, then a flow to a generic point will land us on a fixed point which may enjoy different symmetries. For instance, the theory shown in (2.50) will not generally have a left-right reflection symmetry when the fibers are tuned to give non-trivial gauge groups. However, a Higgs branch flow to an infrared theory with trivial Higgs branch yields a $(2,0)$ theory, which does have a $\mathbb{Z}_{2}$ reflection symmetry.

More generally, we see that in a Higgs branch flow to an infrared theory with trivial Higgs branch, there is a match between the automorphisms of the base lattice, and the automorphisms of the physical theory. This is simply because in such situations, the minimal resolution of the endpoint configuration of curves dictates the resolved geometry of the base, and no tuning of complex structure moduli takes place in this procedure.

\section{Tensor branch moduli space}

Starting from one choice of consistent vevs for the $t^{i}$, it is natural to ask whether there is a group action akin to what is found for the $(2,0)$ theories. This turns out to be far more subtle in the case of $(1,0)$ theories, and we will encounter various generalizations which 
depend both on the nature of the blowups and how we identify the Weyl group and the outer automorphisms of the system.

There are various ways in which we can decompose the automorphism group into a collection of "outer automorphisms" and a Weyl group action, to write

$$
\operatorname{Aut}(\Lambda)=\mathcal{O}_{H} \ltimes \mathcal{W}_{H}
$$

Here, we have included a subscript $H$ to remind us that the particular choice of decomposition we take will be dictated by the ambient values of the complex structure moduli.

Geometrically, the group action $\mathcal{O}_{H}$ corresponds to isometries in the base of the Ftheory model. Since all such bases are resolutions of orbifold singularities of the form $\mathbb{C}^{2} / \Gamma_{\mathrm{U}(2)}$ for $\Gamma$ a finite subgroup of $\mathrm{U}(2)$, the behavior of this group action can be studied by working in the asymptotic limit far from the actual singularity. The group action $\mathcal{W}_{H}$ instead parameterizes redundancies in our resolution, namely they generate discrete gauge transformations.

Let us now turn to the structure of the tensor branch moduli space. Since we are always considering blowups of a singularity of the form $\mathbb{C}^{2} / \Gamma_{\mathrm{U}(2)}$ for $\Gamma$ a finite subgroup of $\mathrm{U}(2)$, the divisors $\alpha_{i}$ with intersection pairing $\alpha_{i} \cap \alpha_{j}=-A_{i j}$ define generators for the Mori cone of effective divisors, which we write as: ${ }^{2}$

$$
\mathcal{C}_{\text {Mori }}=\left\{t^{i} \alpha_{i} \mid t^{i} \geq 0\right\}
$$

Dual to the Mori cone is the Kähler cone:

$$
\mathcal{C}_{\text {Kähler }}=\left\{t_{i} \omega^{i} \mid t_{i} \geq 0\right\}
$$

where we have introduced two-forms $\omega^{i} \in H_{\text {cpct }}^{1,1}(\mathcal{B})$ with compact support which satisfy:

$$
\int_{\alpha_{j}} \omega^{i}=\delta_{j}^{i}
$$

The Kähler form for the base $\mathcal{B}$ is:

$$
J=t_{i} \omega^{i}
$$

Observe that the inverse of the intersection pairing appears via:

$$
\left(A^{-1}\right)^{i j}=-\int_{\mathcal{B}} \omega^{i} \wedge \omega^{j} .
$$

Alternatively, we can introduce generators:

$$
\omega_{i}=A_{i j} \omega^{j}
$$

so we can instead present the Kähler form as:

$$
J=t^{i} \omega_{i}
$$

\footnotetext{
${ }^{2}$ We thank A. Grassi and D.R. Morrison for helpful discussions on this point.
} 
The line element for the metric on the tensor branch moduli space is given by:

$$
A_{i j} \delta t^{i} \delta t^{j}=\int_{\mathcal{B}} \delta J \wedge \delta J
$$

The Kähler cone is specified by $t_{i} \geq 0$, whereas the Mori cone has $t^{i} \geq 0$.

In physical terms, we need to demand that all string tensions are non-negative, namely $t_{i} \geq 0$. We refer to this as the fundamental domain for the moduli space:

$$
\mathbb{D}_{0}=\left\{t_{i} \geq 0\right\}
$$

Observe that our positive definite matrix $A_{i j}$ has inverse $\left(A^{-1}\right)^{i j}$ with all entries positive. This in turn means that if $t_{i} \geq 0$ for all $i$, we also have:

$$
t^{i}=\left(A^{-1}\right)^{i j} t_{j} \geq 0
$$

so in this sense, the physical moduli space is fully specified by positivity in the Kähler cone. This is somewhat different from the F-theory construction of supergravity theories, where both positivity in the Kähler cone and Mori cone must be simultaneously imposed [94].

Consider next the group action of $\operatorname{Aut}(\Lambda)$ on the physical theory. In an "active frame," we interpret the $\omega_{i}$ as elements of the vector space $H_{\text {cpct }}^{2}(\mathcal{B}, \mathbb{R})$ which transform as:

$$
\omega_{i} \mapsto \sigma_{i}^{j} \omega_{j}
$$

A complementary picture which is most convenient for our present purposes is to instead adopt a "passive frame" in which the coordinates themselves transform, namely:

$$
t_{j} \mapsto\left(\sigma^{-1}\right)_{j}^{i} t_{i},
$$

with the transpose $\sigma^{T}$ acting on the dual coordinates $t^{i}$. Consequently, the chambers of the physical moduli space are swept out by the orbits of $\mathbb{D}_{0}$ under the group action by $\operatorname{Aut}(\Lambda)$.

Now in the case of the $(2,0)$ theories, acting by the automorphism group leads to a tessellation of the extended moduli space. For example, the physical moduli space of vacua is given by

$$
\mathcal{M}_{(2,0) \text { theory }}=\mathbb{R}^{5 T} / \mathcal{W}
$$

Viewed as a $(1,0)$ theory, we can write the tensor branch moduli space as: $\mathbb{R}^{T} / \mathcal{W}$. Indeed, starting from $\mathbb{D}_{0}$, we produce all of the other chambers through the orbits of the Weyl group action. In this description, the group $\mathcal{O}_{\mathrm{ADE}}$ specify discrete isometries of the chamber. That is, they should be viewed as a discrete global symmetries of the system. ${ }^{3}$

Turning next to the $(1,0)$ theories, we can now ask a quite similar question concerning the orbit of the discrete gauge symmetries $\mathcal{W}_{H}$, as defined in line (3.1). In particular, we would like to know whether we can expect to tessellate the moduli space, or whether there are forbidden regions of $\mathbb{R}^{T}$ which appear in no orbit.

\footnotetext{
${ }^{3}$ One might ask whether it is possible to gauge these discrete symmetries, thus generating new examples of $(2,0)$ theories. The spectrum of local operators would be the same, but the spectrum of extended objects would be different. We do not appear to have this freedom in string constructions, so this symmetry would appear to be anomalous in the field theory. It would nevertheless be interesting to verify this explicitly.
} 
To aid us in our analysis of this question, we note that the existence of such phenomena is fully determined by the automorphism group. Indeed, even though the actual geometry of the moduli space will depend on the precise decomposition Aut $(\Lambda)=\mathcal{O}_{H} \ltimes \mathcal{W}_{H}$, the $\mathcal{O}_{H}$ never flip the signs of the Kähler moduli; they act as discrete isometries on the fundamental domain $\mathbb{D}_{0}$. Consequently, they simply map the chamber back to itself, and we are free to consider the full group action by $\operatorname{Aut}(\Lambda)$ in determining the orbit of $\mathbb{D}_{0}$.

Suppose, then, that we have two lattices $\Lambda$ and $\Lambda^{\prime}$ related as in line (2.18):

$$
L: \Lambda^{\prime} \rightarrow \Lambda
$$

so that automorphisms of the two lattices are related via the transformation:

$$
L^{-1} \cdot \sigma \cdot L=\sigma^{\prime}
$$

Precisely because each automorphism maps to another, we see that the corresponding group action on $\mathbb{R}^{T}$ will be related by conjugation by $L$, viewed now as a linear map:

$$
L: \mathbb{R}^{T} \rightarrow \mathbb{R}^{T} .
$$

By construction, this linear map has trivial kernel i.e. it is invertible.

Our plan in this section will be to analyze the variety of phenomena which we can expect in the extended Kähler cone. First, we establish that in theories where the endpoint is either trivial or given by just a collection of -2 curves, there is a tessellation of $\mathbb{R}^{T}$ via orbits of the fundamental domain. In all other cases, however, we find that the resulting structure of moduli space is more intricate. We find that when the endpoint contains at least one curve of self-intersection $-n$ for $n>2$, that there are "forbidden zones" in $\mathbb{R}^{T}$ develop which cannot be reached by any element of $\mathcal{W}$.

\subsection{Tessellating $\mathbb{R}^{T}$}

In this section we study tensor branches which produce a tessellation of $\mathbb{R}^{T}$ via orbits of $\mathbb{D}_{0}$ under the group action of $\operatorname{Aut}(\Lambda)$. To begin, we suppose that we have managed to find a lattice which admits a tessellation of $\mathbb{R}^{T}$. In this situation, the tensor branch moduli space will be

$$
\mathcal{M}=\mathbb{R}^{T} / \mathcal{W}
$$

in the obvious notation. Next, suppose that we have another lattice $\Lambda^{\prime}$ related to this one by a change of basis:

$$
L: \Lambda^{\prime} \rightarrow \Lambda \text {. }
$$

Since the generators of the two automorphisms map to one another, we know that:

$$
\operatorname{Aut}(\Lambda) \simeq \operatorname{Aut}\left(\Lambda^{\prime}\right)
$$

and moreover, the orbits of the fundamental domains $\mathbb{D}_{0}$ and $\mathbb{D}_{0}^{\prime}$ also map to one another. Consequently, the orbits also match, and a tessellation for one theory determines a tessellation for the other. Note that in general, however, the resulting orbits could have quite different structure. 
We now show that all theories with trivial endpoint or an endpoint with just -2 curves produce a tessellation of $\mathbb{R}^{T}$. Consider first the case of a trivial endpoint. After $T$ blowups, we always reach the same automorphism group:

$$
\operatorname{Aut}\left(\mathbb{Z}^{\oplus T}\right)=S_{T} \ltimes\left(\mathbb{Z}_{2}\right)^{T} .
$$

Now, in the case of independent blowups, namely a collection of -1 curves which do not intersect, the intersection form is proportional to the identity matrix. In this case, the $S_{T}$ factor acts as an outer automorphism, and is clearly responsible for permuting the different -1 curves. For this theory of $T$ independent E-strings, the tensor branch moduli space is

$$
\mathcal{M}_{T \text { E-strings }}=\underbrace{\mathbb{R} / \mathbb{Z}_{2} \times \ldots \times \mathbb{R} / \mathbb{Z}_{2}}_{T}
$$

where the $S_{T}$ acts as a permutation on the different factors. This clearly yields a tessellation of $\mathbb{R}^{T}$.

Contrast this with the case of the $1,2, \ldots, 2$ configuration, in which there are no outer automorphisms. In this situation, all of the automorphisms are discrete gauge symmetries, and the Weyl group is just that of the Lie algebra $\mathfrak{s p}_{T}$. We again get a tessellation of moduli space, but the structure of the moduli space is quite different:

$$
\mathcal{M}_{\text {Rank } T \text { E-string }}=\mathbb{R}^{T} / \mathcal{W}\left(\mathfrak{s p}_{T}\right) \text {. }
$$

Additional examples include all of the conformal matter theories. For example, the theories with $G \times G$ global symmetry are given by the configurations of curves:

$$
\begin{aligned}
& D_{N} \times D_{N}: 1 \\
& E_{6} \times E_{6}: 1,3,1 \\
& E_{7} \times E_{7}: 1,2,3,2,1 \\
& E_{8} \times E_{8}: 1,2,2,3,1,5,1,3,2,2,1 .
\end{aligned}
$$

In all of these cases, we expect the left/right symmetry to actually be a discrete gauge symmetry of the tensor branch. To see why, it is helpful to consider other blowup patterns, such as the configurations of curves:

$$
1, \frac{1}{4}, 1 \text { and } \quad 1, \frac{1}{5}, 1
$$

These respectively admit an $S_{3}$ and $S_{4}$ symmetry. However, these are not really global symmetries, since they can be viewed as permutations present in the theory of $T-1$ independent E-strings in which the common $E_{8}$ flavor symmetry has been gauged. Indeed, this interpretation is compatible with the fact that there is no normal subgroup $\mathcal{W}$ of $\operatorname{Aut}(\Lambda)$ such that $\operatorname{Aut}(\Lambda) / \mathcal{W}$ is given by these would be "outer automorphisms".

Consider next the case of endpoints with just -2 curves. If we perform no blowups, then we simply have the standard ADE Weyl group action on $\mathbb{R}^{T}$. We can also perform blowups, in which case we again get a tessellation of $\mathbb{R}^{T}$. 


\subsection{Forbidden zones}

For more general endpoint configurations, we find that the group action on the fundamental domain does not yield a tessellation of $\mathbb{R}^{T}$. It could happen that there are certain points of $\mathbb{R}^{T}$ which lie in no orbit of the automorphism group.

We now establish that forbidden zones occur whenever we have at least two curves in the endpoint configuration, one of which has self-intersection $-n$ with $n>2$. Denote by $\Lambda_{\text {end }}$ the corresponding lattice. To establish this, we recall that the automorphism group $\operatorname{Aut}\left(\Lambda_{\text {end }}\right) \varsubsetneqq \operatorname{Aut}(\Lambda)$ is a proper subgroup of the one we would obtain by replacing our $-n$ curve by a -2 curve. Here, $\Lambda$ denotes the lattice obtained by replacing all curves with self-intersection less than -2 by -2 curves, namely an A- or D-type root lattice.

Consequently, $\mathbb{R}^{T}$ can be decomposed into the Weyl chambers generated by $\mathcal{W} \subset \operatorname{Aut}(\Lambda)$, the corresponding Weyl group. Indeed, the Weyl group acts transitively on these Weyl chambers so we know that there is actually a one to one correspondence between elements of the Weyl group $\mathcal{W}$ and these chambers.

But precisely because the group action on the $t$ is the same for elements of $\operatorname{Aut}\left(\Lambda_{\text {end }}\right)$ and $\operatorname{Aut}(\Lambda)$, we see that the orbits swept out by $\operatorname{Aut}\left(\Lambda_{\text {end }}\right)$ will necessarily be a proper subset of those swept out by $\operatorname{Aut}(\Lambda)$. Consequently, we conclude that we cannot tessellate $\mathbb{R}^{T}$. In fact, we can also identify the forbidden zones: They are all the images generated by elements $\sigma \in \Lambda \backslash \Lambda_{\text {end }}$. The full forbidden zone is then given by:

$$
\mathbb{D}_{\text {forbidden }}=\bigcup_{\sigma \in \Lambda \backslash \Lambda_{\text {end }}} \sigma\left(\mathbb{D}_{0}\right) \text {. }
$$

Note that some of these orbits may have common points in the closure other than the origin. The number of connected components in the moduli space is simply the order of $\left|\mathcal{W}_{\text {end }}\right|:$

$$
\left|\operatorname{Orbit}_{\Lambda_{\text {end }}}\left(\mathbb{D}_{0}\right)\right|=\left|\mathcal{W}_{\text {end }}\right| .
$$

To illustrate the above considerations, it is helpful to now study a few examples. Consider, for example, an endpoint configuration such as 3,3 or 7,7. In this case, the automorphism group of the endpoint is $\mathbb{Z}_{2} \ltimes \mathbb{Z}_{2}$, and the analog of the Weyl group is $\mathbb{Z}_{2}$. Labelling the moduli as $t_{1}$ and $t_{2}$, the orbit of the fundamental domain is:

$$
\text { Orbit }_{\Lambda_{\text {end }}}\left(\mathbb{D}_{0}\right)=\left\{t_{1}, t_{2}>0\right\} \cup\left\{t_{1}, t_{2}<0\right\} .
$$

By inspection, the forbidden zone is:

$$
\mathbb{D}_{\text {forbidden }}=\left\{t_{1}>0 ; t_{2}<0\right\} \cup\left\{t_{1}<0 ; t_{2}>0\right\} .
$$

As a somewhat more involved example, consider an endpoint configuration such as 3,2 . Labelling the modulus of the -3 curve by $t_{1}$ and that of the -2 curve by $t_{2}$, we now have that the orbit of the fundamental domain is:

$$
\begin{aligned}
& \operatorname{Orbit}_{\Lambda_{\text {end }}}\left(\mathbb{D}_{0}\right)=\left\{t_{1}, t_{2}>0\right\} \cup\left\{t_{1}, t_{2}<0\right\} \\
& \cup\left\{t_{1}+t_{2}>0 ; t_{2}<0\right\} \cup\left\{t_{1}+t_{2}<0 ; t_{2}>0\right\} .
\end{aligned}
$$

The forbidden zone is:

$$
\mathbb{D}_{\text {forbidden }}=\left\{t_{1}+t_{2}<0 ; t_{1}>0\right\} \cup\left\{t_{1}+t_{2}>0 ; t_{1}<0\right\} .
$$




\section{Compactification}

So far, our analysis has focused on the formal structure of Green-Schwarz automorphisms, and in particular, their role in dictating the geometry of the tensor branch moduli space. Much as in the case of the $\mathcal{N}=(2,0)$ theories, it is natural to expect that these automorphisms are also important in compactifications to lower-dimensional systems.

Now, as we have already remarked, there is a natural sense in which the automorphisms organize into discrete gauge and global symmetries. In this sense, we can always introduce a decomposition of the automorphism group as

$$
\operatorname{Aut}(\Lambda)=\mathcal{O}_{H} \ltimes \mathcal{W}_{H}
$$

Again, we have introduced the subscript $H$ to indicate that this decomposition depends on the Higgs branch moduli of the physical theory. There are then two separate effects we would like to trace in any compactified theory.

First, there is the impact of the discrete gauge symmetries associated with the factor $\mathcal{W}_{H}$. Roughly speaking, this factor controls the geometry of the moduli space of vacua. Additionally, in configurations with non-trivial deformations to $\mathcal{N}=1$ theories, these symmetries play the role of Seiberg-like duality transformations between IR theories.

Second, there is the impact of the global symmetries $\mathcal{O}_{H}$. Another aim of this section will be to deduce necessary consistency conditions for "discrete quotient" by these symmetries. In the context of $(1,0)$ theories, this procedure can be carried out in a variety of dimensions, and is in weakly coupled settings associated with the presence of various orientifold planes. The local Gauss' law constraint can sometimes also require additional branes to be present. Let us emphasize that this appears to be a distinct notion from the case of adding discrete twist lines to a class $\mathcal{S}$ theory, this being more associated with adding a chemical potential for the discrete symmetry. ${ }^{4}$

Our plan in this section is as follows. For specificity we focus on the special case of compactifications of the class $\mathcal{S}_{\Gamma}$ theories $[6,45,62]$. The discrete gauge symmetries of the $6 \mathrm{D}$ theory lead, for $4 \mathrm{D}$ vacua to Seiberg-like dualities, and in $2 \mathrm{~d}$ vacua lead to twisted sectors labelled by conjugacy classes of the discrete gauge symmetries. For the global symmetries, gauging in lower dimensions also leads to new lower-dimensional theories obtained from "discrete quotients" of the original construction. To study consistent ways to perform such quotients, we focus on the geometric realization afforded by F-theory compactification to consistently track the effects in both the base and fiber of the model.

\subsection{Descendants of the 6D Weyl group}

We now turn to the effects of the analog of the Weyl group in compactifications of $6 \mathrm{D}$ SCFTs. We first consider the case of $4 \mathrm{D}$ theories obtained from compactification on a Riemann surface, and then turn to $2 \mathrm{~d}$ theories obtained from compactification on a four-manifold.

\footnotetext{
${ }^{4}$ We thank T.T. Dumitrescu for helpful discussions on this point.
} 


\subsubsection{D theories}

As a first class of examples, we consider the impact of discrete gauge transformations on the structure of compactified theories. To set the stage, it is helpful to have in mind the case of $N$ M5-branes probing an A-type singularity $\mathbb{C}^{2} / \mathbb{Z}_{k}$. As is by now well-known, this leads, on the tensor branch, to an F-theory model in which the base is:

$$
\left[\mathfrak{s u}_{k}\right] 2, \stackrel{\mathfrak{s u}_{k}}{2}, \stackrel{\mathfrak{s u}_{k}}{2}, \ldots, \stackrel{\mathfrak{s u}_{k}}{2}, \stackrel{\mathfrak{s u}_{k}}{2}\left[\mathfrak{s u}_{k}\right]
$$

a theory with $N-1$ tensor multiplets. In this case, the automorphism group is given by

$$
\operatorname{Aut}(\Lambda)=\mathbb{Z}_{2} \ltimes S_{N},
$$

with $S_{N}$ the permutation group on $N$ letters which acts via the standard Weyl reflections. In terms of the M5-brane picture, the automorphisms correspond to moving the M5-branes past one another.

Compactifying this tensor branch deformation on a $T^{2}$ yields a $4 \mathrm{D} \mathcal{N}=2$ quiver gauge theory. For a theory with $N-1$ simple gauge group factors, each node has gauge group $\mathrm{SU}(k)$. Furthermore, the matter content of each non-abelian gauge theory factor consists of $F=2 k$ hypermultiplets in the fundamental representation. Consequently, we also have a superconformal field theory in four dimensions. In this case, the motion of the M5-branes is rather trivial, and leads us back to the same theory. We can, however, consider various mass deformations as we descend to four dimensions. Additionally, we can tilt the branes by effectively activating a non-trivial superpotential deformation for the Coulomb branch scalar of the $\mathcal{N}=2$ vector multiplet. This sort of operation, and the resulting duality cascades [95] were considered in reference [79] where it was found that the Weyl group transformations then generate a sequence of Seiberg-like dualities [96] as we flow from the UV to the IR.

Assuming that we have generated an appropriate $\mathcal{N}=1$ theory with general ranks for the gauge groups, the reason for a Seiberg-like duality is as follows. Consider the 7branes wrapped over the -2 curves. In this setup, the resulting homology class for all the 7-branes is

$$
[\Sigma]=k_{1} \alpha_{1}+\ldots+k_{N-1} \alpha_{N-1},
$$

where the $\alpha_{i}$ denote simple roots and $k_{i}-1$ is the rank of each SU factor. Upon applying a Weyl group transformation on the $i^{\text {th }}$ node (assuming it is in the middle of the quiver), we have the transformation (see e.g. [79]):

$$
\begin{aligned}
\alpha_{i} & \rightarrow-\alpha_{i} \\
\alpha_{i+1} & \rightarrow \alpha_{i+1}+\alpha_{i} \\
\alpha_{i-1} & \rightarrow \alpha_{i-1}+\alpha_{i},
\end{aligned}
$$

and the coefficient multiplying $\alpha_{i}$ shifts to $k_{i-1}+k_{i+1}-k_{i}$, i.e., $F_{i}-k_{i}$, where $F_{i}$ is the number of flavors in the fundamental representation.

Given this, it is quite natural to ask whether there is an analogous Seiberg-like duality for $4 \mathrm{D}$ theories obtained from M5-branes probing a D- or E-type singularity. Again, we observe that with no mass deformations switched on, permuting the M5-branes simply takes 
us back to the same $4 \mathrm{D} \mathcal{N}=2$ SCFT. We can, of course, entertain $\mathcal{N}=1$ deformations, as well as deformations which break conformal symmetry. We expect that in this broader context, there is a natural generalization of Seiberg duality now using compactifications of 6D conformal matter. From the perspective of F-theory compactification, one complication is that now, the charges of seven-branes are mutually non-local, so the abelian transformation rule given above must be modified. We leave the development of this intriguing possibility for future work.

\subsubsection{D theories}

Another way in which these discrete gauge symmetries show up is in compactification to two dimensions. Indeed, as has been appreciated in string constructions, Seiberg-like dualities, as realized by brane maneuvers can be extended to a variety of dimensions. Some caution is warranted, however because the quantum dynamics in the infrared can be quite different depending on the dimensionality of the resulting theory.

Along these lines, we can also consider the compactification of 6D SCFTs on fourmanifolds. In the case of $(1,0)$ theories on a Kähler surface, this yields a class of $2 \mathrm{~d}$ theories with $\mathcal{N}=(0,2)$ supersymmetry [63, 97, 98]. Compactification of the tensor branch leads to a class of theories known as "DGLSMs" [63], which are a generalization of a $(0,2)$ gauged linear sigma model in which the gauge couplings are now dynamical fields. Orbits of the Weyl group on the tensor branch moduli space descend to non-trivial transformations on the gauge couplings of a DGLSM. Additionally, we know that these orbits serve to also define additional twisted sector states.

The first point is that in the special case where there is a tessellation of the extended Kähler cone as $\mathbb{R}^{T}$ so that the physical tensor branch moduli space is $\mathbb{R}^{T} / \mathcal{W}$, we immediately recognize that the dynamical gauge couplings of the compactified theory generate twisted sectors labelled by the conjugacy classes of $\mathcal{W}$. This holds for blowups of the trivial endpoint as well as all admissible blowups of the ADE endpoints composed of -2 curves. In those cases where we do not have such a tessellation, we anticipate additional strongly coupled phenomena to be present, for example, possible singularities as in the case of conifold points in a conformal field theory. We have already classified the 6D SCFTs where forbidden zones can occur, so we have a clear indication about when to expect such strongly coupled phenomena.

Finally, we expect that the notion of dualities naturally extends to trialities [99-101]. Here again, we expect the discrete gauge symmetries of the $6 \mathrm{D}$ tensor branch to characterize at least part of this structure.

\subsection{Discrete quotients of a $6 \mathrm{D}$ SCFT}

So far, we have focused on the discrete gauge symmetries inherited from the automorphism group. The global symmetries of the 6D also impact the theory, and its compactifications. In compactifications of the $(2,0)$ theory, adding a background chemical potential for this symmetry along a one-cycle is sometimes referred to as introducing a "discrete twist", (see e.g. [1, 80-82]). There is a conceptually separate notion of quotienting by this symmetry to reach a wholly different theory. In perturbative string theory terms, this is associated with adding orientifolds. In this section we shall be interested in this operation. 
Our plan in this subsection will be to determine necessary conditions for quotienting by such discrete symmetries. From the perspective of an F-theory model, we need to ensure that the symmetry present in the isometries of the base extends consistently to the fibers of the model. This means that the full quotient will involve changing both the total number of gauge groups, as well as the specific gauge groups and matter content present in a given generalized quiver.

Already in six dimensions, we can see that such quotients can lead to interesting effects. Now, in the case of the $\mathcal{N}=(2,0)$ theories, we do not expect to generate any theories other than the ADE type ones because all of these models have a purely geometric realization in terms of IIB on an ADE singularity. Once we include various dynamical 7-branes, however, we can also expect to incorporate both $O 7^{-}$-planes and $\mathrm{O}^{+}$-planes. Whereas in F-theory the $\mathrm{O7}^{-}$-planes are fully captured by elliptically fibered Calabi-Yau threefolds, the case of $\mathrm{O}^{+}$-planes involves "frozen" singularities [83-85], and this can generate a small number of additional 6D SCFTs [24]. In reference [86] a formal quotienting procedure was proposed to explain such models. The full set of consistency conditions in this case have yet to be worked out, but we can already see that this leads to the expected structure in lower-dimensional theories.

In compactifications to lower-dimensional systems, we can extract additional consistency conditions. For concreteness, we focus on the case of compactifications to fourdimensional vacua with $\mathcal{N}=2$ supersymmetry. That means we confine our attention to compactification on a $T^{2}$, with possible quotients also included. For concreteness, we focus on compactifications of class $\mathcal{S}_{\Gamma}$ theories, since in such cases the analysis is particularly tractable. Mild deformations of this case can also be extracted from the general considerations we present.

As we lack a worldsheet construction of F-theory, quotienting by these discrete symmetries will instead be pieced together through complementary features. The key idea we shall make use of is that fiber-base duality of the F-theory geometry can lead to a priori distinct 4D theories which nevertheless share a common geometric origin [49] (see also [86, 102]). By construction, we retain all compact two-cycles, so we expect that upon compactification, the dimensions of the Coulomb branches in theories where we interchange base and fiber will still match. Indeed, as we already remarked for theories without a discrete quotient, compactifications of the class $\mathcal{S}_{\Gamma}$ theories at the conformal point take us to affine ADE type quiver gauge theories, and on the partial tensor branch, take us to generalized quivers. Each of these theories flows to a $4 \mathrm{D} \mathcal{N}=2 \mathrm{SCFT}$, and due to their common geometric origin, they have the same Coulomb branch geometry and identical superconformal indices [6]. These common features mean that we also expect discrete quotients to persist on both sides, yielding again a pair of $4 \mathrm{D} \mathcal{N}=2$ SCFTs. Note that this analysis does not require the dimension of the Higgs branches to match, since in the process of taking appropriate decoupling limits, the number of compact three-cycles (and thus the number of Higgs branch moduli) could a priori be different. ${ }^{5}$

\footnotetext{
${ }^{5}$ Indeed, for a genuine duality, we ought to also be able to match the dimensions of the Higgs branches on both sides, since this is in turn related to the values of the conformal anomalies $a$ and $c$.
} 
Now, precisely because the Coulomb branches match, we can track the effects of a discrete quotient in both theories. If we can perform a consistent quotient on both sides, it is strong evidence that the automorphism of the base actually extends to the full Calabi-Yau threefold geometry. Whereas the discrete quotients of the partial tensor branch deformation involves various exceptional group structures, we see that in the affine quiver theory, we always have SU gauge groups and the quotient will generate another classical group, namely $\mathrm{SU}, \mathrm{SO}$ or Sp. Our first task is therefore to consistently identify which of the affine quivers can be quotiented, and to then match this data to their counterparts involving a discrete quotient on the partial tensor branch side.

To guide us in our analysis of quotienting the affine quiver gauge theories, we note that since this operation does not involve introducing a mass scale, we expect the quotient to also be a superconformal field theory. For the quivers obtained using classical gauge groups and matter, this proves to be quite restrictive, and often leads to a discrete set of possibilities. In the associated string construction, these constraints are interpreted in terms of a local Gauss' law constraint for the Ramond-Ramond charge. Consequently, the quotienting by the discrete symmetry typically involves the presence of both an orientifold plane and D-branes.

The quotient of an affine quiver gauge theory leads us to a restricted set of $4 \mathrm{D} \mathcal{N}=2$ SCFTs. In particular, based on fiber-base duality of the associated F-theory geometry, we expect that if it exists, there is a corresponding discrete quotient of the partially tensor branch deformation. In this case, the string construction involves non-perturbative sevenbranes, so aside from the A-type class $\mathcal{S}_{\Gamma}$ theories, we expect that the quotient will involve a non-perturbative generalization of orientifold planes, i.e. another choice of seven-branes. We can, nevertheless, piece together the structure of the resulting theory by appealing to the form of the associated affine quiver gauge theory.

Since we shall be using the same conditions repeatedly, it is helpful to collect some general remarks about orientifold projections of the affine quiver gauge theories in one location. For such theories, a perturbative string theory analysis is available. For example, the orientifold projection acts on an A-type symmetric quiver by folding it, as illustrated in figure 1. We note that when the number of curves present in the small resolution is odd, then the orientifold action naturally fixes one curve of the geometry, and an additional node of the corresponding affine quiver is also held fixed. In the case where the number of curves is even, then all curves of the geometry are interchanged, but the node present in the affine extension is held fixed. This is all in accord with the partial tensor branch description, where we have a marked curve in the associated $I_{n}$ Kodaira-Tate fiber, associated with the zero section.

Moreover, this orientifold projection maps the SU gauge groups to either SO or Sp groups, depending on the projection. Since we demand a construction consistent with an open string construction, large $N$ scaling already dictates the basic form of these maps:

$$
\begin{aligned}
& \mathrm{SU}(N) \stackrel{\mathrm{SO}}{\longmapsto} \mathrm{SO}\left(N+\delta_{\mathrm{SO}}\right) \\
& \mathrm{SU}(N) \stackrel{\mathrm{Sp}}{\longmapsto} S p\left(\frac{N-\delta_{\mathrm{Sp}}}{2}\right),
\end{aligned}
$$


Tensor Branch Phase

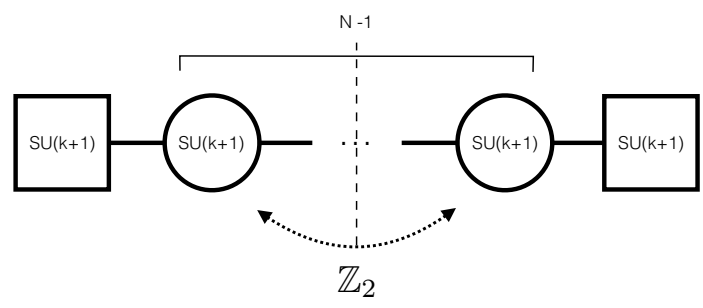

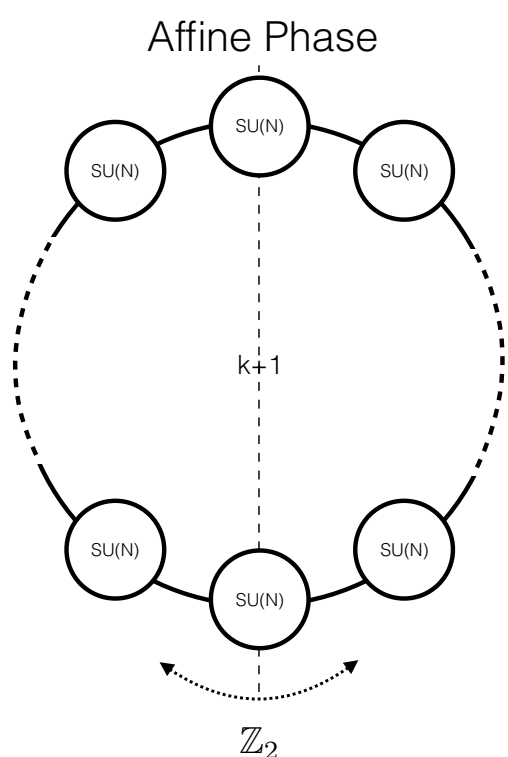

Figure 1. The two quiver phases of A-type class $\mathcal{S}_{\Gamma}$ theories compactified on a $T^{2}$. In addition, the effect of quotienting by the global symmetries associated to the outer automorphisms is shown. On the left the reduction on the tensor branch yields $(N-1) \mathrm{SU}(k+1)$ gauge nodes and two $\mathrm{SU}(k+1)$ flavor symmetry factors. On the right the affine quiver with $(k+1) \mathrm{SU}(N)$ gauge nodes. The segments represent standard $\mathcal{N}=2$ hypermultiplets in the bifundamental representation.

namely the rank decreases by a factor of two, with a possible small shift as captured by the presence of the $\delta$ 's. In the stringy construction, this is due to the fact that the orientifolds also carry Ramond-Ramond charge, and this in turn alters the ranks of gauge groups.

Now, our primary focus in this work will be to match various $\mathbb{Z}_{2}$ discrete quotients of the affine quiver phase to corresponding discrete quotients on the partial tensor branch. For some examples relevant in the context of 6D SCFTs and their compactification, see for example $[56,103,104]$. We also note that there are various orientifold projections which one can in principle adopt for affine quivers. As such, we should not expect to find a single orientifold of an affine quiver, but several possibilities. This is borne out by the fact that if we only impose the conditions of conformal invariance (by also including suitable flavors), then we can actually find various sequences of gauge groups which all lead to conformal fixed points. The fact that there are multiple choices suggests a richer structure in which we could also attempt to match various Higgs branch flows. Here, we shall confine our analysis to a few examples in which we can verify a candidate pair of theories. Indeed, this suffices for our present purpose, since all we really wish to demonstrate is that a discrete quotient of the partial tensor branch exists. For this reason, we shall find it convenient to adopt a "bottom up" approach where we impose constraints from conformal invariance, and the dimension of the Coulomb branch, both for the affine quiver phase, and the partial tensor branch phase. 


\begin{tabular}{|l|l|l|l|}
\hline$G$ & $\mathrm{SU}(k)$ & $\mathrm{Sp}(k)$ & $\mathrm{SO}(k)$ \\
\hline$r_{G}$ & $k-1$ & $k$ & {$[k / 2]$} \\
\hline $\operatorname{Ind}(\mathbf{A d j})$ & $k$ & $2 k+2$ & $k-2$ \\
\hline $\operatorname{Ind}(\mathbf{f u n d})$ & $\frac{1}{2}$ & 1 & 1 \\
\hline $\operatorname{Ind}($ anti) & $\frac{k-2}{2}$ & $2 k-2$ & $k-2$ \\
\hline $\operatorname{Ind}(\mathbf{s y m m})$ & $\frac{k+2}{2}$ & $2 k+2$ & $k+2$ \\
\hline $\operatorname{dim}($ fund) & $k$ & $2 k$ & $k$ \\
\hline
\end{tabular}

Table 1. Relevant group theory data

\subsection{1 $4 \mathrm{D} \mathcal{N}=2$ SCFTs and generalized quivers}

In preparation for our analysis of discrete quotients, in this subsection we collect some general comments on the types of quiver gauge theories and generalizations we can expect to encounter. Our guiding principle is to seek out various ways to generate a $4 \mathrm{D}$ conformal fixed point from a $6 \mathrm{D}$ theory compactified on a $T^{2}$. Some such theories have been studied for example in $[46,49,54,58,105]$.

We mainly focus on compactifications of the class $\mathcal{S}_{\Gamma}$ theories, namely those obtained from $N$ M5-branes probing an ADE singularity. Compactification on a $T^{2}$ yields an SCFT. There are various orders of limits one can take, depending on whether we compactify the 6D SCFT, or instead a tensor branch deformation of this theory. We describe each in turn in what follows.

In the case where we remain at the $6 \mathrm{D}$ conformal fixed point, compactification on a $T^{2}$ leads us to the theory of $N$ D3-branes probing an ADE singularity. This is described by a quiver gauge theory with gauge groups $\mathrm{SU}\left(d_{i} N\right)$, and $d_{i}$ the Dynkin labels of the associated affine Dynkin diagram of ADE type [106-108]. We also have bifundamental hypermultiplets between each node, as dictated by the structure of the Dynkin diagram.

As is well-known, this yields a class of $\mathcal{N}=2$ SCFTs. Indeed, in our conventions, the beta function coefficient for an $\mathcal{N}=2$ gauge theory with classical gauge group with $F$ hypermultiplets in the fundamental representation is:

$$
\begin{aligned}
& b(\mathrm{SU}(M))=2 M-F \\
& b(\mathrm{SO}(M))=2(M-2)-2 F \\
& b(\operatorname{Sp}(M))=2(2 M+2)-2 F
\end{aligned}
$$

where in the above, we have also included the SO and Sp gauge groups as we shall need them later. ${ }^{6}$ Observe that for all the affine Dynkin diagram nodes with gauge group $\mathrm{SU}\left(d_{i} N\right)$, we have $F=2 d_{i} N$, so we indeed realize a $4 \mathrm{D} \mathcal{N}=2 \mathrm{SCFT}$. For the ranks and group theory data of the various perturbative gauge groups see table 1 .

Another way to realize a $4 \mathrm{D} \mathcal{N}=2 \mathrm{SCFT}$ with such gauge groups is to construct a linear chain of gauge groups. We will find this sort of structure appearing repeatedly in

\footnotetext{
${ }^{6}$ Recall that in our conventions, $\mathfrak{s p}(1) \simeq \mathfrak{s u}(2)$.
} 
our analysis of both the original and quotiented theories, so we collect the relevant points here as well. One such set of examples is given by taking all of the ranks to be fixed and equal as follows:

$$
[\mathrm{SU}(M)]-\mathrm{SU}(M)-\ldots-\mathrm{SU}(M)-\mathrm{SU}(M)-\mathrm{SU}(M)-\ldots-\mathrm{SU}(M)-[\mathrm{SU}(M)],
$$

where we have indicated a flavor symmetry in square brackets on the left and right. Here, each link corresponds to a full hypermultiplet in the bifundamental representation. There is a natural "orientifolded" version of this theory given by replacing the various SU factors by alternating SO and Sp gauge group factors:

$$
\begin{aligned}
& {[\mathrm{SO}(2 a+2)] \stackrel{1 / 2}{-} \mathrm{Sp}(a) \stackrel{1 / 2}{-} \ldots{ }^{1 / 2}-\mathrm{SO}(2 a+2) \stackrel{1 / 2}{-} \mathrm{Sp}(a) \stackrel{1 / 2}{-} \mathrm{SO}(2 a+2) \stackrel{1 / 2}{-} \ldots \stackrel{1 / 2}{-} \mathrm{Sp}(a) \cdot \stackrel{1 / 2}{-}[\mathrm{SO}(2 a+2)]} \\
& {[\mathrm{Sp}(a)]^{1 / 2}-\mathrm{SO}(2 a+2) \stackrel{1 / 2}{-} \ldots{ }^{1 / 2}-\mathrm{Sp}(a) \stackrel{1 / 2}{-} \mathrm{SO}(2 a+2) \stackrel{1 / 2}{-} \mathrm{Sp}(a) \stackrel{1 / 2}{-} \ldots{ }^{1 / 2}-\mathrm{SO}(2 a+2) .{ }^{1 / 2}[\mathrm{Sp}(a)]}
\end{aligned}
$$

where we have indicated the presence of a half hypermultiplet in the bifundamental representation by writing ${ }^{1 / 2}$. This is possible precisely because the bifundamental is in a pseudoreal representation of the product gauge group. It is also possible to combine all three types of gauge group factors in a single quiver. An example of this type is:

$$
[\mathrm{SU}(2)]-\mathrm{SU}(N)-\mathrm{Sp}(N-1) \stackrel{1 / 2}{-} \mathrm{SO}(2 N) \stackrel{1 / 2}{-} \mathrm{Sp}(N-1) \stackrel{1 / 2}{-} \ldots,
$$

There is another way to compactify which yields a class of $4 \mathrm{D} \mathcal{N}=2$ SCFTs. This involves first moving onto the partial tensor branch of the 6D theory, and only then compactifying. Geometrically, we separate the positions of the M5-branes and then compactify to four dimensions. In doing so, we do not move onto the tensor branch of the conformal matter sectors. As shown in references $[46,49,54]$ the conformal matter descends to a $4 \mathrm{D}$ $\mathcal{N}=2$ SCFT which we view as a generalization of the standard $4 \mathrm{D}$ hypermultiplet. Indeed, it enjoys a flavor symmetry $G_{L} \times G_{R}$. The contribution to the beta function coefficient of a $4 \mathrm{D} \mathcal{N}=2$ gauge theory with gauge group $G$ of such conformal matter has been computed in $[46,54]$, and the result is: ${ }^{7}$

$$
b_{G \times G \operatorname{conf}}(G)=-h_{G}^{\vee},
$$

where $h_{G}^{\vee}$ is the dual Coxeter number for the gauge group. Now, the beta function coefficient from the $\mathcal{N}=2$ vector multiplet is:

$$
b_{\mathrm{vec}}(G)=2 h_{G}^{\vee}
$$

so we see that coupling each gauge group to precisely two such conformal matter sectors leads to a $4 \mathrm{D} \mathcal{N}=2 \mathrm{SCFT}[46,54]$.

\footnotetext{
${ }^{7}$ A note on normalization conventions. In [46, 54], the contribution to the beta function coefficient from an $\mathcal{N}=2$ vector multiplet is given as $4 h_{G}^{\vee}$, with $h_{G}^{\vee}$ the dual Coxeter number of the group $G$. For $\mathrm{SU}(N)$, this yields $4 N$ as opposed to the "standard" convention of $2 N$ used in the weakly coupled literature.
} 
Hence, one can now see that we obtain two a priori different 4D $\mathcal{N}=2$ SCFTs from the same class $\mathcal{S}_{\Gamma}$ theory. In the case of the compactified tensor branch deformation, each node supports the group $G \times \mathrm{U}(1)$, where the $\mathrm{U}(1)$ comes from reduction of the tensor multiplet to four dimensions. Though such $\mathrm{U}(1)$ factors flow to weak coupling in the infrared, the full match of the Coulomb branch dictated by the geometry means we ought to include these factors in our analysis. Indeed, because of their common origin in F-theory in which we retain all compact two-cycles, the resulting $4 \mathrm{D}$ theories must have the same Coulomb branch dimension [6, 49].

The notion of conformal matter can also be generalized beyond ADE gauge groups. For example, given a subgroup $K \subset G$ with imbedding index $I_{K: G}$, we can also compute the contribution to the beta function of this subgroup:

$$
b_{G \times G \operatorname{conf}}(K)=-I_{K: G} \times h_{G}^{\vee} .
$$

Another generalization along these lines is the compactification of the rank $Q$ E-string theory on a $T^{2}$. This yields the rank $Q$ Minahan-Nemeschansky theory, which enjoys an $E_{8}$ flavor symmetry. Weakly gauging this group, the contribution to the beta function is [109]:

$$
b_{\mathrm{MN}[Q]}\left(E_{8}\right)=-6 Q \text {. }
$$

Finally, we note that compactifying the completely resolved tensor branch deformation of a $6 \mathrm{D}$ SCFT need not generate an SCFT. Instead, we must keep the conformal matter at the origin of its tensor branch. To illustrate this point, consider the case of a linear quiver with $\mathrm{SO}(8)$ gauge groups, with $D_{4} \times D_{4}$ conformal matter between each gauge group. Geometrically, this conformal matter is generated by a collapsing -1 curve. If we also resolve this -1 curve, then compactification of the fully resolved tensor branch will produce $\mathrm{SO}(8)$ gauge groups coupled to no matter fields, and therefore confines in the infrared. Indeed, to obtain a $4 \mathrm{D}$ SCFT, we must keep the conformal matter at the origin of the tensor branch, namely, we only compactify the partial tensor branch deformation.

\subsubsection{A-type $\mathcal{S}_{\Gamma}$ theories}

Let us begin with discrete quotients of the A-type class $\mathcal{S}_{\Gamma}$ theories. These are realized by $N$ M5-branes probing the transverse geometry $\mathbb{R}_{\perp} \times \mathbb{C}^{2} / \mathbb{Z}_{k}$, where the total number of tensors is $T=N-1$. Recall that the tensor branch obtained from separating the M5-branes is given by the 6D F-theory model:

$$
\left[\mathfrak{s u}_{k}\right] \underbrace{\stackrel{\mathfrak{s u}_{k}}{2,}, \mathfrak{S u}_{k}, \ldots, \mathfrak{s u}_{k}, \mathfrak{s u}_{k}}_{N-1}\left[\mathfrak{s u}_{k}\right] .
$$

Compactification on a $T^{2}$ yields a $4 \mathrm{D} \mathcal{N}=2$ quiver gauge theory where each gauge group factor is $\mathrm{SU}(k) \times \mathrm{U}(1)$. The abelian factors all flow to weak coupling in the infrared, but the non-abelian factors support a non-trivial conformal fixed point.

The outer automorphism group of the base is a $\mathbb{Z}_{2}$ symmetry which amounts to a reflection about the midpoint of this diagram. We distinguish four different possibilities, depending on whether the axis of reflection holds fixed a gauge group or a conformal matter 
link (in this case a weakly coupled 6D hypermultiplet), and whether the $\mathrm{SU}(k)$ gauge groups have odd or even rank. These choices are captured by $k$ and $N$ even or odd.

To determine the effects of the $\mathbb{Z}_{2}$ quotient of the tensor branch deformation, we shall now use fiber-base duality to study the closely related quiver gauge theories obtained from compactification of the $6 \mathrm{D}$ conformal fixed point. Recall that in the absence of a $\mathbb{Z}_{2}$ quotient, the $4 \mathrm{D}$ theory so obtained is given by the circular quiver gauge theory of $\mathrm{SU}(N)$ gauge groups:

$$
/ / \underbrace{\mathrm{SU}(N)-\ldots-\mathrm{SU}(N)-\ldots-\mathrm{SU}(N)}_{k+1} / /
$$

where the notation // indicates that we join the left and right by a hypermultiplet in the bifundamental representation. By inspection, we see that the $\mathbb{Z}_{2}$ symmetry of the tensor branch is also present for this class of theories. We also see that there is again a natural distinction between the four distinct choices presented by taking $k$ and $N$ even or odd.

Since in this case the tensor branch admits a weakly coupled description, we shall find it convenient to also make use of the weakly coupled IIA brane construction of these theories. In this case, the sequence of gauge groups given in line (4.21) are specified by a collection of NS5-branes for the links with D6-branes suspended between them for the gauge groups. When we compactify on a $T^{2}$, it is more appropriate to T-dualize this configuration to that of NS5-branes with D4-branes suspended between each pair. Applying a $\mathbb{Z}_{2}$ quotient of both sides amounts to introducing an orientifold plane. For O6-planes, there are two general variants one can consider (see e.g. $[84,110-112]$ given by the $O 6^{-}$or $O 6^{+}$-plane. Recall that under a $\mathrm{RR}(p+1)$-form in which the Dp-brane carries +1 units of charge, an $O p^{-}$plane carries $-2^{p-5}$ units of charge and an $O p^{+}$plane carries $+2^{p-5}$ units of charge, other variants being unavailable for O6-planes. Now, on the tensor branch side of the construction, we see that our quotient can therefore only locally satisfy Gauss' law if we introduce an $\mathrm{O6}^{-}$-plane and two D6-branes. We shall indeed find that this is compatible with the "bottom up" condition of conformal invariance.

The reduction of the tensor branch quiver to $4 \mathrm{D}$ can be described via the suspended configuration of D4-NS5 branes, where the D4 are extended along $x^{6}$, with a system of $\mathrm{O}^{-}+2 \mathrm{D} 6$ sitting at $x^{6}=0$, as shown in figure 2 . The total brane charge of O6 ${ }^{-}+2 \mathrm{D} 6$ is zero, and hence the Gauss' law constraint associated with the charges of these objects is locally satisfied. There are four distinct possibilities to consider, depending on whether $k$ and $N$ are respectively even or odd. We shall therefore step through each possibility in what follows. The basic idea will be to first consider the D6-branes and $\mathrm{O6}^{-}$-plane all on top of each other, and passing through either the D4-brane (in the case of $N$ even) or the NS5-brane (in the case of $N$ odd). Moving the D6-branes away from this fixed locus will then take us to the other possible theories, i.e. the cases of $k$ even and odd. In figure 2, we display also other cases depending on whether $k$ and $N$ are even or odd. For these cases consistency requires moving the $\mathrm{O}^{-}+2 \mathrm{D} 6$ stack on top of an NS5, or to move an NS5 brane inside the $\mathrm{O}^{-}+2 \mathrm{D} 6$ system. The affine quiver theory can be described by the same suspended brane configuration, where now $x_{6}$ is a compactified $S^{1}$ direction with two O6 $^{-}$ $+2 \mathrm{D} 6$ systems at the opposite ends of the circle. For each case in the tensor branch phase 


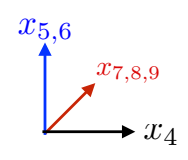

k-odd

$\mathrm{N}$-even

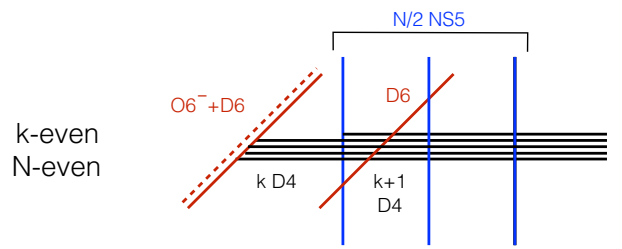

$(\mathrm{N}+1) / 2$ NS5

k-even

$\mathrm{N}$-odd

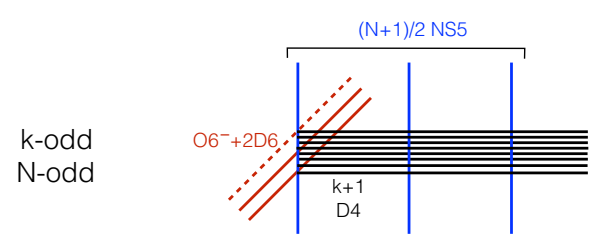

Tensor Branch Phase
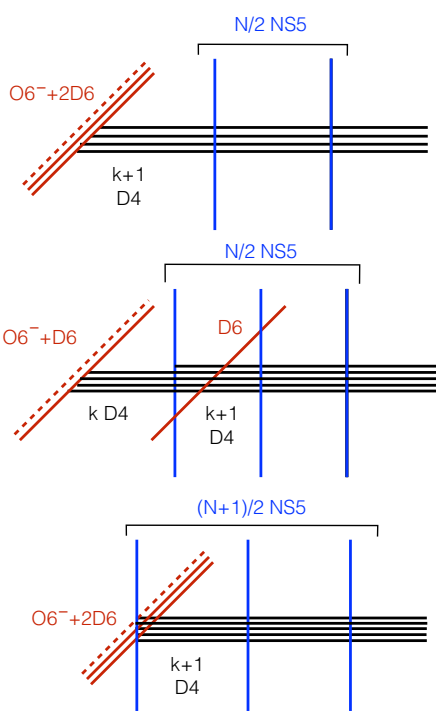

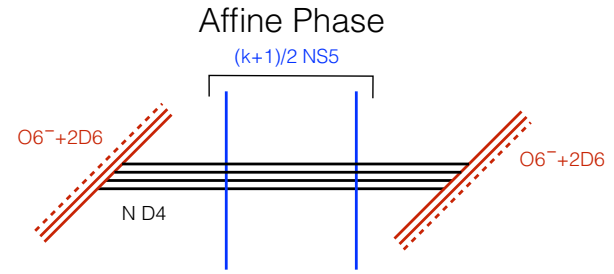

$(k+2) / 2$ NS5

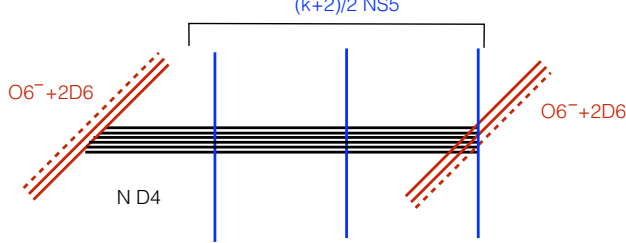

$(k+2) / 2$ NS5

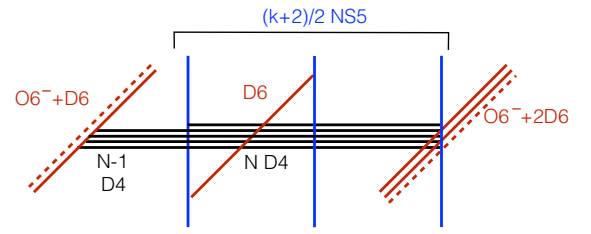

$(k+1) / 2$ NS5

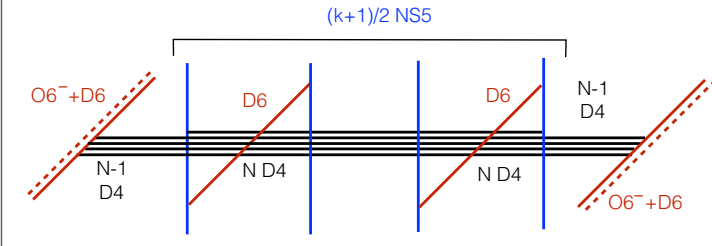

Figure 2. Suspended brane configurations for discrete quotients of the A-type class $\mathcal{S}_{\Gamma}$ theories on the tensor branch. We depict linear symmetric suspended branes configurations of D4 (in black) filling $x^{0}, \ldots, x^{3}$ and extended along $x_{6}$, NS5 branes (in blue) filling $x^{0}, \ldots, x^{3}$, wrapping $x^{4}, x^{5}$ (i.e. the two torus directions), and probing $x^{6}, \mathrm{O}^{-}+2 \mathrm{D} 6$ (in red) filling $x^{0}, \ldots, x^{3}$ and extended along $x_{7}, x_{8}, x_{9}$. The other configurations are given by moving the $\mathrm{O}^{-}+2 \mathrm{D} 6$ on top of an NS5 brane, or by moving one NS5 brane inside the $\mathrm{O}^{-}+2 \mathrm{D} 6$ system depending on whether $k$ and $N$ are even or odd. In this figure, only the physical branes have been illustrated. However in the presence of orientifold $\mathrm{O6}^{-}$there is an equivalent mirror image of the brane system, which in the affine cases makes the quiver circular, and the $x_{6}$ direction compact.

we have a corresponding affine brane system. It is important to notice that in figure 2 only the physical branes are shown (namely no images under the orientifold are included).

The theories associated with the brane system of figure 2 are given in figure 3. Conformal invariance dictates that only Sp groups are allowed. For instance, If we replace the Sp with SO groups, either the beta function for SO or the one for the close SU group would have a negative value, and hence the quiver would not be conformal. Note that this is compatible with the fact that we have $\mathrm{O6}^{-}$-planes rather than $\mathrm{O6}^{+}$-planes.

Moreover, having two D6's on top of the $\mathrm{O}^{-}$plane is consistent with the flavor symmetries being $\mathrm{SU}(2)$ or $\mathrm{U}(1) \times \mathrm{U}(1)$. Finally, the dimensions of the Coulomb branches are summarized in table 2 . 


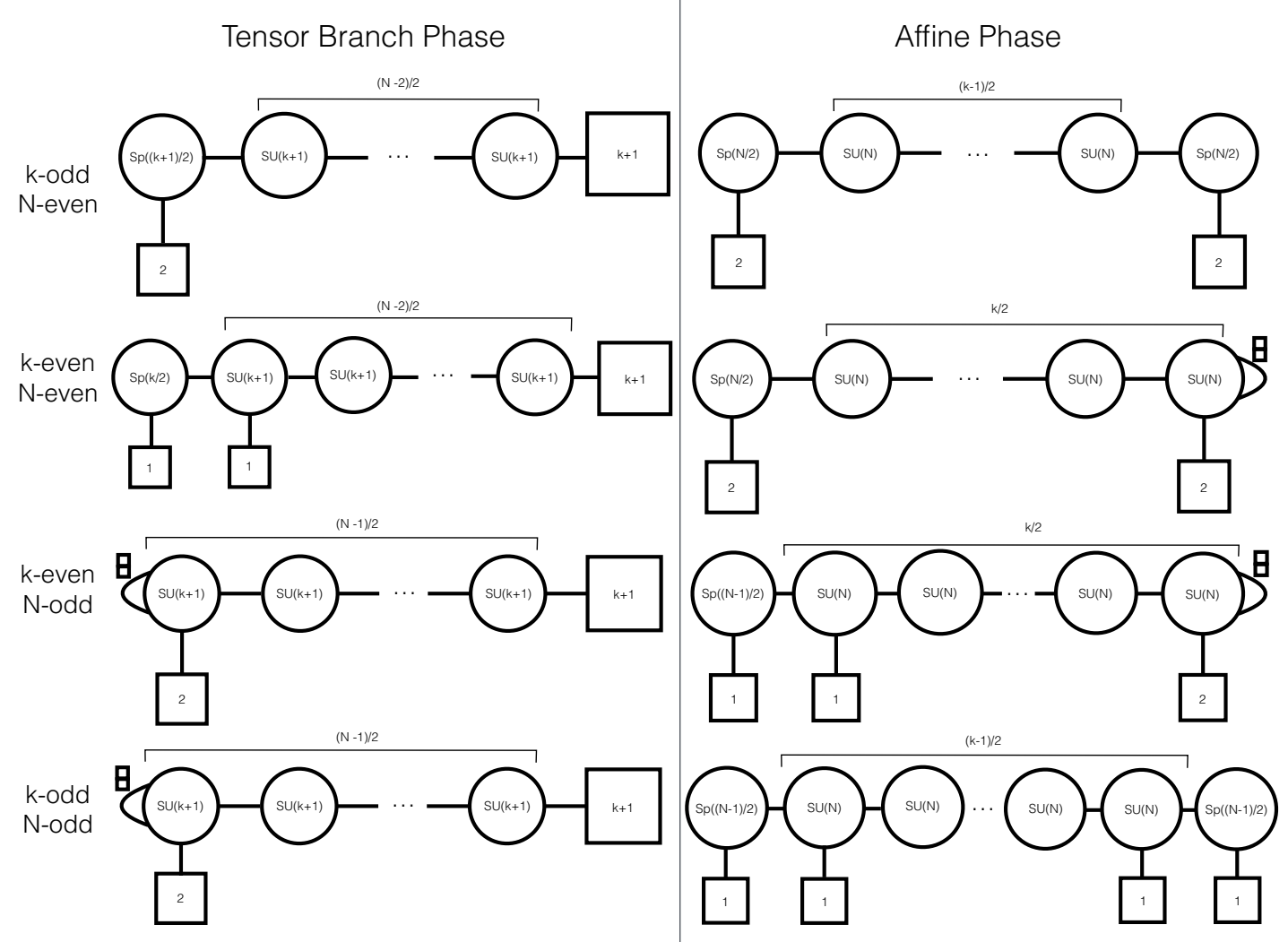

Figure 3. Resulting quiver theories for A-type class $\mathcal{S}_{\Gamma}$ theories associated with the brane systems in 2. On the left the reduction on the tensor branch to $4 \mathrm{D}$, on the right the reduction at the fixed point. The possible cases are listed depending on $k$ and $N$ even or odd. The double box labeling some matter on the right or left gauge nodes stands for a full antisymmetric hypermultiplet.

\begin{tabular}{|l|l|l|}
\hline & $N$ Even & $N$ Odd \\
\hline$k$ Even & $\frac{k N-k+N}{2}$ & $\frac{k N-k+N-1}{2}$ \\
\hline$k$ Odd & $\frac{k N-k+N+1}{2}$ & $\frac{k N-k+N-1}{2}$ \\
\hline
\end{tabular}

Table 2. Dimensions of the Coulomb branch of the various cases

\subsubsection{D-type $\mathcal{S}_{\Gamma}$ theories}

We now turn to discrete quotients of the D-type class $S_{\Gamma}$ theories. Let us begin by reviewing the general structure of the D-type theories before quotientng. These are realized by $N$ M5-branes probing a D-type singularity. Here the total number of tensors is $T=2 N-1$. We shall denote this singularity as $D_{k}$ where we label according to the associated Dynkin diagram with $k \geq 4$ nodes obtained from a small resolution of the singularity. We move onto the partial tensor branch by separating all the M5-branes. In this phase, each M5brane defines a $6 \mathrm{D}$ conformal matter sector in the sense of references $[6,40]$. The partial 
tensor branch is described in the F-theory geometry by:

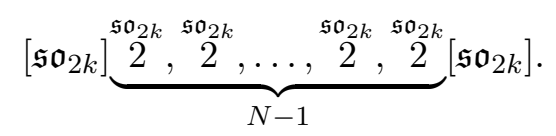

By inspection, there is again a $\mathbb{Z}_{2}$ reflection symmetry by which we can quotient the theory. Compactification on a $T^{2}$ takes us to a $4 \mathrm{D} \mathcal{N}=2 \mathrm{SCFT}$. As explained in reference [46, 54], the $6 \mathrm{D}$ conformal matter contributes the requisite amount to maintain conformal invariance of the generalized quiver gauge theory. Indeed, in our normalization conventions, we have two $D \times D$ conformal matter sectors, and a pair of such sectors precisely cancels the contribution to the SO beta functions from the $\mathcal{N}=2$ vector multiplets. This results in a $4 \mathrm{D} \mathcal{N}=2$ SCFT using generalized quivers. Now, the Coulomb branch of such theories is calculated by including the contributions from both the vector multiplets as well as the conformal matter sectors. Observe that in contrast to the A-type case, the matter sectors now contribute non-trivially to the $4 \mathrm{D}$ Coulomb branch, since in the F-theory construction they involve a -1 curve with an $\mathfrak{s p}_{k-4}$ gauge algebra over each such curve. If we instead compactify the $6 \mathrm{D}$ fixed point, we obtained a classical quiver gauge theory with gauge groups:

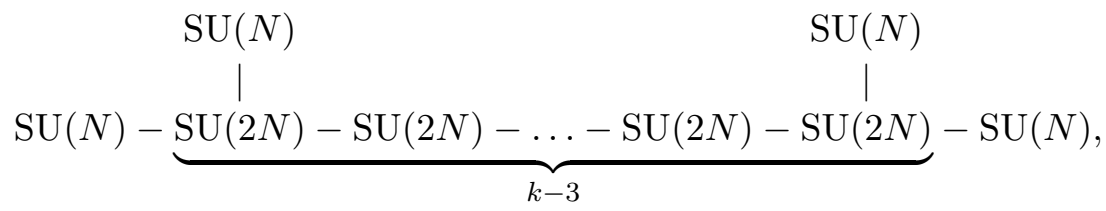

which again realizes a $4 \mathrm{D} \mathcal{N}=2 \mathrm{SCFT}$.

By inspection, both phases enjoy a discrete $\mathbb{Z}_{2}$ symmetry, so we do expect to be able to consistently gauge this symmetry. One complication is that now on the tensor branch side, we must specify this quotient on either the $\mathfrak{s o}_{2 k}$ factor or the conformal matter link. The former case occurs when $N$ is even while the latter occurs when $N$ is odd.

To facilitate our understanding of these cases, we first study the proposed group action on the affine quiver gauge theories. Here, we see that the central spine has a fixed plane, so we expect an $\mathrm{SU}(N)$ factor on the left and right of the quotient of line (4.24), with the fixed spine composed of an alternating sequence of $\mathrm{SO}$ and Sp gauge group factors. Again, conformal invariance of the entire configuration severely limits the available possibilities. For example, in the interior of the quotiented spine of line (4.24), the gauge groups must alternate as

$$
\ldots \stackrel{1 / 2}{-} \mathrm{Sp}(M-1) \stackrel{1 / 2}{-} \mathrm{SO}(2 M) \stackrel{1 / 2}{-} \mathrm{Sp}(M-1) \stackrel{1 / 2}{-} \mathrm{SO}(2 M) \stackrel{1 / 2}{-} \ldots
$$

Additionally, the leftmost and rightmost $\mathrm{SU}(2 N)$ factors must both be Sp gauge group factors. If they are $\mathrm{SO}$ gauge groups instead, we find that the $\mathrm{SU}(N)$ gauge group factors have too much matter to support a conformal fixed point. This in turn limits us to the special case of $k$ even. For $k$ odd, we do not find a consistent $\mathbb{Z}_{2}$ quotient. The resulting 
quotient leads us to the following quiver gauge theory:

$[\mathrm{SU}(2)]-\mathrm{SU}(N)-\underbrace{\mathrm{Sp}(N-1) \stackrel{1 / 2}{-} \mathrm{SO}(2 N) \stackrel{1 / 2}{-1 / 2} \mathrm{SO}(2 N) \stackrel{1 / 2}{-} \mathrm{Sp}(N-1)}_{k-3}-\mathrm{SU}(N)-[\mathrm{SU}(2)]$.

Let us note that there is another phase compatible with conformal invariance in which we alternate $\mathrm{Sp}(N)$ and $\mathrm{SO}(2 N+2)$ gauge groups. In this case, the flavors move to the leftmost and rightmost Sp factors. Anticipating what we shall find on the partial tensor branch and the dimension of its Coulomb branch, we shall focus on the case of line (4.26) in what follows. In this case, the complex dimension of the Coulomb branch is:

$$
\operatorname{dim}_{\mathbb{C}}(\text { Coulomb })=N k-N-\frac{k}{2}-1 .
$$

Consider next the quotient of the partial tensor branch. Based on our analysis of the affine quivers, we confine our attention to $k$ even, but with $N$ arbitrary. There are thus two cases to analyze, depending on whether a gauge group or conformal matter sector is held fixed under the group action.

To start, suppose that an $\mathrm{SO}(2 k)$ gauge group is held fixed under the group action. We would like to determine the resulting gauge group from the quotient $\mathrm{SO}(2 k) / \mathbb{Z}_{2}$. Now, in an F-theory compactification, there is a well-known effect known as "monodromy" which amounts to applying a quotient on the quotiented affine Dynkin diagram. For example, this quotient realizes an $\mathrm{Sp}(N)$ gauge group from an $\mathrm{SU}(2 N)$ gauge theory, and produces an $\mathrm{SO}(2 k-1)$ gauge group from an $\mathrm{SO}(2 k)$ gauge theory.

The general lesson we would like to extract from our analysis of the A-type theories, as well as the D-type affine quiver phase is that the $\mathbb{Z}_{2}$ quotient has two general effects on the partial tensor branch. First, it appears to introduce a low rank flavor symmetry, that is, one which does not scale with the values $N$ and $k$. Additionally, the rank of a gauge group fixed by the quotient reduces by roughly $1 / 2$ rather than a constant shift. We must perform such a reduction in the rank of the gauge group in order to match the dimension of the Coulomb branch to that present in the affine quiver phase. This is all compatible with the perturbative type IIA construction of such gauge theories, where we would introduce an additional $\mathrm{O6}^{-}$-plane. The point is that in general, this quotient is a distinct notion from monodromy of the fiber present in an F-theory construction.

Taking this into account, we conjecture that the quotient of the partial tensor branch, is, for the case of a fixed $\mathrm{SO}(2 k)$ gauge group, given by:

$$
[\mathrm{SO}(2)] \stackrel{1 / 2}{-} S p\left(\frac{k-2}{2}\right) \underset{[\mathrm{Sp}(1) \times \mathrm{Sp}(1)]}{\mathrm{CM}} \underbrace{\mathrm{SO}(2 k) \stackrel{\mathrm{CM}}{-} \mathrm{SO}(2 k) \ldots \stackrel{\mathrm{CM}}{-} \mathrm{SO}(2 k)}_{(N-2) / 2} \stackrel{\mathrm{CM}}{-}[\mathrm{SO}(2 k)],
$$

where we have introduced the notation $\stackrel{\text { CM }}{-}$ to denote the dimensional reduction on a $T^{2}$ of six-dimensional $\mathrm{SO}(2 k) \times \mathrm{SO}(2 k)$ conformal matter. This also includes the special case of conformal matter between the Sp and SO factor at the left. Here, we have gauged the $\mathrm{Sp}((k-2) / 2)$ subgroup of $\mathrm{SO}(2 k)$, with the commutant remaining as a global symmetry. 
This is necessary to have the correct amount of matter contribute to both the Sp and SO gauge coupling beta functions. The relevant embedding of subalgebras is:

$$
\mathfrak{s o}(2 k) \supset \mathfrak{s p}\left(\frac{k}{2}\right) \times \mathfrak{s p}(1) \supset \mathfrak{s p}\left(\frac{k-2}{2}\right) \times \mathfrak{s p}(1) \times \mathfrak{s p}(1) .
$$

Using this, we can calculate the contribution to the $\operatorname{Sp}((k-2) / 2)$ beta function coefficient from the corresponding one for $D_{k} \times D_{k}$ conformal matter:

$$
b_{D_{k} \times D_{k} \operatorname{conf}}\left(\operatorname{Sp}\left(\frac{k-2}{2}\right)\right)=-h_{\mathrm{SO}(2 k)}^{\vee}=-(2 k-2) .
$$

The total $\operatorname{Sp}\left(\frac{k-2}{2}\right)$ beta function coefficient is then given by adding up the contributions from the conformal matter sector, the vector multiplet, and $F$ hypermultiplets, which we take to be in the fundamental representation (in accord with the string construction). The result is:

$$
b_{\text {total }}\left(\operatorname{Sp}\left(\frac{k-2}{2}\right)\right)=2 h_{\mathrm{Sp}((k-2) / 2)}^{\vee}-2 h_{\mathrm{SO}(2 k)}^{\vee}-2 F=2 k-(2 k-2)-2 F=2-2 F,
$$

so we must add a half hypermultiplet in the fundamental representation of an $\mathrm{SO}(2)$ flavor symmetry, as indicated in line (4.28). As a further piece of evidence in favor of our conjecture for the quotient, we can also calculate the dimension of the Coulomb branch, and it indeed matches that of line (4.27); we have $N / 2 D_{k} \times D_{k}$ conformal matter sectors, each with Coulomb branch dimension $k-3,(N-2) / 2 \mathrm{SO}(2 k) \times \mathrm{U}(1)$ gauge groups, and one $\mathrm{Sp}\left(\frac{k-2}{2}\right) \times \mathrm{U}(1)$ gauge group. The total Coulomb branch dimension is therefore:

$$
\operatorname{dim}_{\mathbb{C}}(\text { Coulomb })=\frac{N}{2}(k-3)+\frac{N-2}{2}(k+1)+\frac{k}{2}=N k-N-\frac{k}{2}-1,
$$

which precisely matches the dimension of the Coulomb branch in the affine quiver phase! Note that if we had not reduced the rank of the leftmost Sp gauge group by a factor of $1 / 2$, the dimension of the quotiented tensor branch phase would have been greater than that of the affine quiver. A related point is that if we had instead attempted to use an $\mathrm{SO}(k)$ gauge group rather than $\mathrm{Sp}((k-2) / 2)$, we would have encountered an $\mathrm{SO}(k)$ flavor symmetry, contradicting the requirement that the flavor symmetry remains independent of $k$ and $N$. As a final additional comment, we note that for this construction to be valid, we require $k$ to be even, a point we already encountered in the affine quiver phase.

Consider next the case of $N$ even, namely where the $\mathbb{Z}_{2}$ quotient holds fixed a conformal matter link. Returning to the affine quiver phase, we see no distinction between the cases $N$ even and odd. This strongly indicates that the quotient should also make sense in the discrete quotient of the partial tensor branch. In this case, however, the fixed locus of the quotient will be a conformal matter sector. Now, for A-type conformal matter, namely weakly coupled hypermultiplets, we can see that a bifundamental is instead replaced by a two-index anti-symmetric or symmetric representation (depending on the type of orientifold plane) of a single SU gauge group.

We shall now conjecture a generalization of such matter fields, as dictated by consistency with the requirements that we have a $4 \mathrm{D}$ SCFT, that the dimension of the Coulomb 

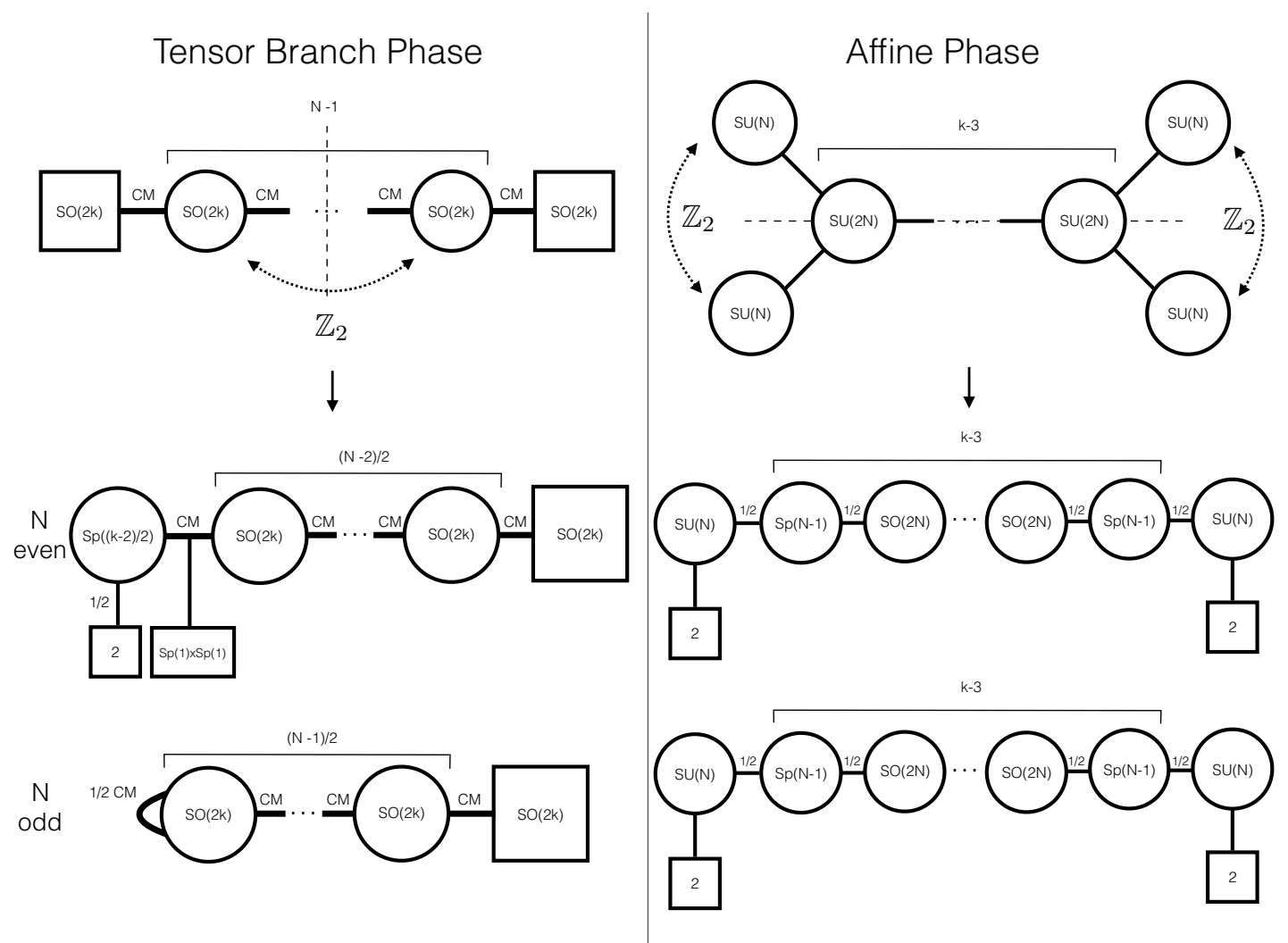

Figure 4. D-type quiver theories. On top the unquotiented theories in the tensor branch phase (left) and affine phase (right). On the bottom the two cases depending on $N$ being even or odd for the two corresponding phases. The segments represents standard $\mathcal{N}=2$ hypermultiplets, when the $1 / 2$ appears on a link it stands for half hypermultiplet. The label "CM" means that it is not just an hypermultiplet, but generalized matter coming from compactification of the conformal matter given by the two connected gauge nodes.

branch matches that of the affine quiver phase, and that any flavor symmetries visible in the UV do not scale with the parameters $k$ and $N$.

To determine the related structure for D-type conformal matter, we first consider the case of $D_{k} \times D_{k}$ conformal matter compactified on a $T^{2}$, in which the two $\mathrm{SO}(2 k)$ factors are flavor symmetries. If, instead of gauging both such factors, we only gauge the diagonal, we obtain in four dimensions, an $\mathrm{SO}(2 k)$ gauge theory where the $4 \mathrm{D}$ conformal matter contributes double that of a $D_{k} \times D_{k}$ conformal matter sector. The reason is simply that the embedding index is two for the diagonal subgroup. We denote this system as:

$$
\stackrel{\mathrm{CM}}{\subset} \mathrm{SO}(2 k) \stackrel{\mathrm{CM}}{-} \ldots
$$

in the obvious notation. Observe that we now have effectively three $4 \mathrm{D}$ conformal matter sectors contributing to the $\mathrm{SO}(2 k)$ beta function, so this theory will not flow to a conformal fixed point. If, however, we also assume the existence of a $\mathbb{Z}_{2}$ quotient which acts on the $\stackrel{\mathrm{CM}}{\subset}$ factor, we again have the requisite amount of matter to ensure a fixed point for this 
$\mathrm{SO}(2 k)$ factor. ${ }^{8}$ We denote the resulting generalized quiver as:

$$
\stackrel{\frac{1}{2} C M}{\subset} \underbrace{\mathrm{SO}(2 k) \stackrel{\mathrm{CM}}{-}{ }^{\mathrm{CM}} \mathrm{SO}(2 k)}_{(N-1) / 2} \stackrel{\mathrm{CM}}{-}[\mathrm{SO}(2 k)] .
$$

We should also ask what the resulting contribution is to the dimension of the Coulomb branch. To address this question, it is helpful to briefly pass to the tensor branch of the 6D $D_{k} \times D_{k}$ conformal matter. Recall that this is given by a -1 curve which supports an $\mathfrak{s p}(k-4)$ gauge theory coupled to $4 k$ half hypermultiplets in the fundamental representation. We expect that just as in the other D-type generalized quiver, the $\mathbb{Z}_{2}$ quotient decreases the rank of this gauge group factor by roughly $1 / 2$.

To track in more detail what happens, it is helpful to consider the dimensional reduction of an isolated -1 curve by itself. In six dimensions, collapsing this curve to zero size generates the rank one E-string 6D SCFT. Upon reduction on a circle, we obtain an $\operatorname{Sp}(1)$ gauge theory with $N_{f}=7$ flavors, which at strong coupling enjoys an $E_{8}$ flavor symmetry [113]. We emphasize that geometrically, it is appropriate to view this as an $\mathrm{Sp}(1)$ rather than $\mathrm{SU}(2)$ gauge group in the sense that we have "already quotiented" by the outer automorphism of SU(2). Now, acting once again by a $\mathbb{Z}_{2}$ quotient ought to reduce the rank by one half again. This takes us below a rank one theory, so for this reason we expect this contribution to the Coulomb branch to vanish for this system. Note, however, that there is still a non-trivial contribution to the beta function of the weakly gauged flavor symmetry coming from the matter fields which transformed in the fundamental representation of $\operatorname{Sp}(1)$. Putting this all together, we conclude that the $\frac{1}{2} C M$ sector of type $D_{k}$ will have a Coulomb branch of dimension $(k-4) / 2$.

Though it would of course be desirable to have a systematic derivation of our conjecture, we can already see that it passes a few non-trivial checks. First, we see that we again retain a conformal fixed point. Additionally, the dimension of the Coulomb branch receives contributions from the $(N-1) / 2 \mathrm{SO}(2 k) \times \mathrm{U}(1)$ factors, the $(N-1) / 2 D_{k} \times D_{k}$ $4 \mathrm{D}$ conformal matter sectors, and the single $\frac{1}{2} C M$ sector. Taking all this into account, we obtain a Coulomb branch dimension:

$$
\operatorname{dim}_{\mathbb{C}}(\text { Coulomb })=\frac{N-1}{2}(k+1)+\frac{N-1}{2}(k-3)+\frac{k-4}{2}=N k-N-\frac{k}{2}-1,
$$

which matches the dimension found for the affine quiver phase!

There are, however, a few curious features of this theory. Observe that for $k=4$, the $\frac{1}{2} C M$ sector has no Coulomb branch, but still contributes to the running of the $\mathrm{SO}(2 k)$ gauge coupling. It would be most instructive to better understand this generalized matter sector, either using methods from IIA suspended brane configurations, F-theory, or related compactifications of class $\mathcal{S}$ theories with discrete quotients.

The theories in the tensor branch and affine phases are summarized in figure 4 .

\footnotetext{
${ }^{8}$ Here we allow for the definition of a $\frac{1}{2} C M$ sector to have some number of weakly coupled hypermultiplets in order to ensure conformal invariance of the $\mathrm{SO}(2 k)$ sector.
} 


\subsubsection{E-type $\mathcal{S}_{\Gamma}$ theories}

Let us now turn to the E-type class $\mathcal{S}_{\Gamma}$ theories and their discrete quotients. On the partial tensor branch obtained by separating $N$ M5-branes along the $\mathbb{R}_{\perp}$ factor of the transverse geometry

$$
\mathbb{R}_{\perp} \times \mathbb{C}^{2} / \Gamma,
$$

we have the F-theory realization given by:

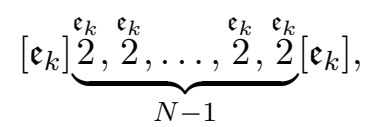

for $k=6,7,8$. Again, there is a $\mathbb{Z}_{2}$ outer automorphism of the configuration, so we can again ask whether this can be consistently gauged upon compactification to four dimensions. To address this issue, we again pass to the affine quiver phase. Now, for the $E_{6}$ case, we see that both the original Dynkin diagram and its affine extension enjoy a $\mathbb{Z}_{2}$ reflection symmetry. In the $E_{7}$ case, we see that only the affine extension of the Dynkin diagram enjoys this reflection symmetry. This is problematic in the context of F-theory constructions, because we typically select an elliptic fibration with a section. The existence of this section selects out the affine node, and breaks the symmetry. For this reason, we do not consider the $E_{7}$ or for that matter, $E_{8}$ cases in what follows.

Let us now study in greater detail the $E_{6}$ case. To begin, we consider the quiver gauge theory of $N$ D3-branes probing an $E_{6}$ singularity (see e.g. [107, 108]):

$$
\begin{gathered}
\mathrm{SU}(N) \\
\mathrm{SU}(2 N) \\
\stackrel{\mathrm{SU}}{\mid}(N)-\mathrm{SU}(2 N)-\mathrm{SU}(3 N)-\mathrm{SU}(2 N)-\mathrm{SU}(N) .
\end{gathered}
$$

Performing a $\mathbb{Z}_{2}$ quotient, we find two possible configurations of gauge groups:

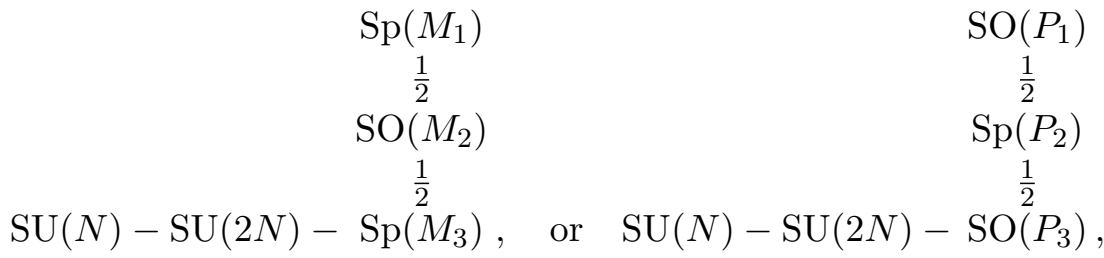

where we have omitted possible flavor symmetry factors.

Let us now demonstrate that the latter case in line (4.39) cannot produce a conformal fixed point. To this end, we note that the condition of conformal invariance requires all beta function coefficients to vanish. Writing out the non-trivial constraints, we have the conditions:

$$
\begin{aligned}
b(\mathrm{SU}(2 N)) & =4 N-N-P_{3}-F_{4} \\
b\left(\mathrm{SO}\left(P_{3}\right)\right) & =2\left(P_{3}-2\right)-4 N-2 P_{2}-2 F_{3} \\
b\left(\mathrm{Sp}\left(P_{2}\right)\right) & =2\left(2 P_{2}+2\right)-P_{3}-P_{1}-2 F_{2} \\
b\left(\mathrm{SO}\left(P_{1}\right)\right) & =2\left(P_{1}-2\right)-2 P_{2}-2 F_{1},
\end{aligned}
$$


where we have referred to the number of flavors of the $\mathrm{SU}(2 N)$ gauge theory as $F_{4}$. Consider, however, the weighted sum:

$$
b_{\text {sum }}=4 b(\mathrm{SU}(2 N))+3 b\left(\mathrm{SO}\left(P_{3}\right)\right)+2 b\left(\mathrm{Sp}\left(P_{2}\right)\right)+b\left(\mathrm{SO}\left(P_{1}\right)\right) .
$$

This sum is negative for any non-negative number of flavors, and is given by:

$$
b_{\text {sum }}=-8-4 F_{4}-6 F_{3}-4 F_{2}-2 F_{1}<0,
$$

so we conclude that this sequence of gauge groups will not produce a conformal fixed point.

Focusing, then, on the remaining case of two Sp factors and a single SO factor, we seek a choice of ranks and flavors which maintains conformal invariance, and which we can compare with a discrete quotients of a partial tensor branch.

As we have already remarked, the possible presence of flavor branes means there are multiple ways to obtain a conformal fixed point. Much as in the A- and D-type theories, this also depends on whether $N$ is even or odd. We present some consistent choices in figure 5. The particular choice we make anticipates the condition that we can match the dimension of the Coulomb branch to one obtained from a discrete quotient of the partial tensor branch. This also depends on whether $N$ is even or odd, and we have in the two cases:

$$
\begin{aligned}
\operatorname{dim}_{\mathbb{C}}\left(\text { Coulomb }_{N \text { even }}\right) & =6 N-1 \\
\operatorname{dim}_{\mathbb{C}}\left(\text { Coulomb }_{N \text { odd }}\right) & =6 N-3 .
\end{aligned}
$$

We remark that there are additional consistent choices which also produce a conformal fixed point. The examples presented here are based on the "bottom up" condition that we match to a candidate discrete quotient of the partial tensor branch. Much as in the case of the A- and D-type cases, we can distinguish between whether $N$ is even or odd, namely whether the discrete quotient holds fixed the gauge group, or the conformal matter sector.

In the case where $N$ is even, our conjecture for the discrete quotient of the partial tensor branch is:

$$
\left[G_{2}\right] \stackrel{\mathrm{CM}}{-} F_{4} \stackrel{\mathrm{CM}}{-} \underbrace{E_{6} \stackrel{\mathrm{CM}}{-} \stackrel{\mathrm{CM}}{-} E_{6}}_{(N-2) / 2} \stackrel{\mathrm{CM}}{-}\left[E_{6}\right] .
$$

In the above, we have identified the $\mathbb{Z}_{2}$ quotient of $E_{6}$ with the gauge group $F_{4}$. Additionally, the conformal matter between the $F_{4}$ and $E_{6}$ groups is the standard $E_{6} \times E_{6}$ conformal matter in which we gauge the $F_{4}$ subgroup (with embedding index one). We also introduced a rank one Minahan-Nemeschansky theory [114] with $E_{8}$ flavor symmetry in which we gauge the $F_{4}$, retaining a $G_{2}$ flavor symmetry. We can calculate the contribution to the $F_{4}$ beta function coefficient from these sectors:

$$
b\left(F_{4}\right)=2 h_{F_{4}}^{\vee}-h_{E_{6}}^{\vee}-6=0,
$$

where the contribution from $2 h_{F_{4}}^{\vee}$ comes from the $F_{4}$ vector multiplet, the contribution $-h_{E_{6}}^{\vee}$ comes from an $\left(E_{6}, E_{6}\right)$ conformal matter sector, and the -6 comes from the rank one Minahan-Nemeschansky theory with a gauged $F_{4} \subset E_{8}$. Based on this, we conclude 


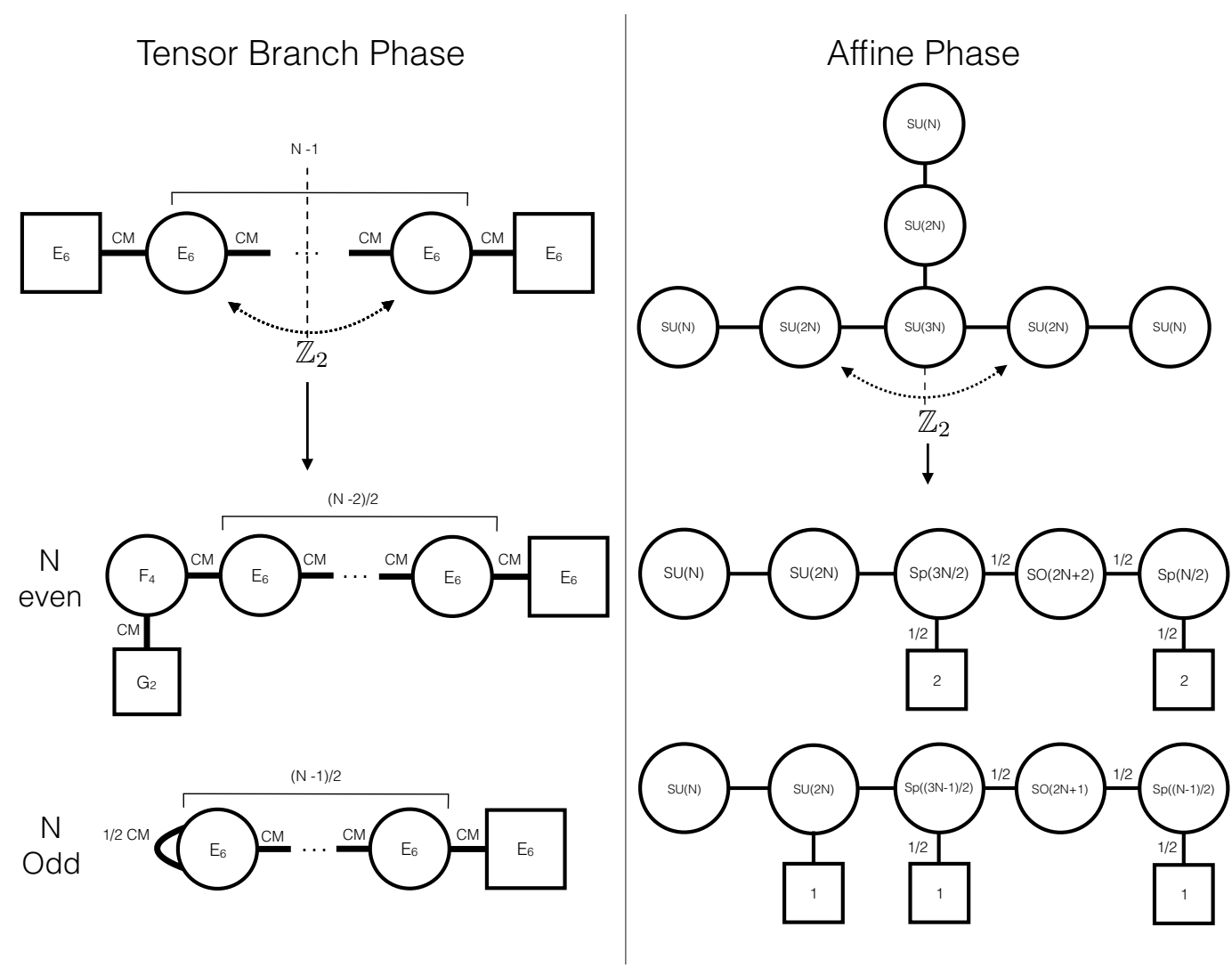

Figure 5. Quiver theories obtained from a discrete quotient of $E_{6}$-type class $\mathcal{S}_{\Gamma}$ theories reduced on a $T^{2}$ with a discrete quotient. On top the unquotiented theories in the tensor branch phase (left) and affine phase (right). On the bottom the two cases depending on $N$ being even or odd for the two corresponding phases. The segments represents standard $\mathcal{N}=2$ hypermultiplets, when the $1 / 2$ appears on a link it stands for half hypermultiplet. The label "CM" denotes generalized matter coming from compactification of the $6 \mathrm{D}$ conformal matter.

that all gauge group factors are indeed conformal. Observe also that the dimension of the Coulomb branch in this case matches that of the affine quiver phase:

$$
\operatorname{dim}_{\mathbb{C}}\left(\text { Coulomb }_{N \text { even }}\right)=6 N-1 .
$$

Let us now turn to the case of $N$ odd. Here, a conformal matter sector will be fixed by the discrete quotient. Now, in the $D_{k} \times D_{k}$ conformal matter case, we argued that the system

$$
\stackrel{\frac{1}{2} C M}{\subset} \mathrm{SO}(2 k) \stackrel{\mathrm{CM}}{-}
$$

would retain conformal invariance. We can provide a similar argument in the $E_{6}$ case for the system:

$$
\stackrel{\frac{1}{2} C M}{\subset} E_{6}{ }^{\mathrm{CM}}
$$

Here we allow for the definition of a $\frac{1}{2} C M$ sector to have some number of weakly coupled hypermultiplets in order to ensure conformal invariance of the $E_{6}$ sector. 
We can also calculate the dimension of the Coulomb branch. For $E_{6} \times E_{6}$ conformal matter, recall that in the resolved phase, we have the tensor branch description:

$$
1, \stackrel{\mathfrak{s u}_{3}}{3}, 1
$$

which reduces in the $4 \mathrm{D}$ theory to a Coulomb branch of dimension five. In anticipation of our discussion of the $\mathbb{Z}_{2}$ quotient, let us now discuss how to count the Coulomb branch for this theory. First of all, each -1 curve contributes a single tensor multiplet. In the ambient Calabi-Yau threefold, each -1 curve is associated with a del Pezzo 9 surface. Additionally, the resolved geometry of an isolated -3 curve consists of an affine $\widehat{A}_{2}$ Dynkin diagram of three intersecting Hirzebruch $\mathbb{F}_{1}$ surfaces $[66,115]$. We thus see five independent divisors, and these translate to a count of $h_{\text {cpct }}^{1,1}=5$ in the local Calabi-Yau geometry.

We now study the $\mathbb{Z}_{2}$ quotient of this theory. By inspection, we see that the two -1 curves and their associated surfaces will be identified. Additionally, in the resolution of the -3 curve theory with three Hirzebruch surfaces, the $\mathbb{Z}_{2}$ symmetry of the affine $\widehat{A}_{2}$ configuration identifies two of the Hirzebruch surfaces, and leaves the other one invariant (but not pointwise invariant). So, out of the original five divisors, only three will be independent in the $\mathbb{Z}_{2}$ quotient. We conclude that the dimension of the Coulomb branch for the $\mathbb{Z}_{2}$ quotient of line (4.53) is:

$$
\operatorname{dim}_{\mathbb{C}}\left(\operatorname{Coulomb}\left[(1,3,3,1) / \mathbb{Z}_{2}\right]\right)=3
$$

Observe that in this case, the -3 curve modulus has not been "projected out", whereas in the D-type conformal matter case we did project out the -1 curve modulus. Roughly speaking, this is due to the fact that $3 / 2>1$, whereas $1 / 2<1$.

Putting all of this together, we conjecture the following generalized quiver in the case $N$ odd:

$$
\stackrel{\frac{1}{2} C M}{\subset} \underbrace{E_{6}{ }^{\mathrm{CM}} \ldots \stackrel{\mathrm{CM}}{-} E_{6}}_{(N-1) / 2} \stackrel{\mathrm{CM}}{-}\left[E_{6}\right],
$$

which has Coulomb branch dimension:

$$
\operatorname{dim}_{\mathbb{C}}\left(\text { Coulomb }_{N \text { odd }}\right)=6 N-3
$$

As a final comment, we note that for the affine quiver phases, the lowest dimension for the Coulomb branch we could find is $6 N-3$, again suggesting that the dimension of the Coulomb branch for the quotiented conformal matter is three rather than two.

Based on this, we conclude that a discrete quotient is possible both for $N$ even and $N$ odd. Again, we stress that we have pieced our analysis together from various complementary points of view. It would be interesting to study related examples and extend the correspondence to all possible choices of orientifolds / discrete quotients.

The theories in the tensor branch and affine phases are summarized in figure 5. 


\section{Conclusions}

In this paper we have calculated the discrete gauge and global symmetries of a $6 \mathrm{D}$ SCFT generated by automorphisms of the lattice of strings present on the tensor branch. These automorphisms capture an important ambiguity in specifying the structure of GreenSchwarz terms. The ambiguity is resolved by specifying a chamber of moduli space. We have also elaborated on the similarities and differences with the case of the $(2,0)$ theories. For example, in the $(1,0)$ case, the resulting moduli space for $T$ tensor multiplets only admits a tessellation of $\mathbb{R}^{T}$ in rather special circumstances. More generally, the orbit of the fundamental domain of moduli space may lead to "forbidden zones". Finally, we have also taken some preliminary steps in identifying the role of the automorphism group in compactifications. In the remainder of this section, we discuss some potential avenues for future investigation.

One of the features which would be quite interesting to understand better is Seiberg-like dualities for $\mathcal{N}=1$ gauge theories with exceptional gauge groups and conformal matter. As we have already noted, the primary complication here is that the transformation rules will need to involve a non-abelian generalization of the familiar rule $\mathrm{SU}(N) \mapsto \mathrm{SU}(F-N)$. We anticipate that the geometric realization in F-theory will provide a route to understand this structure.

We have also seen that the structure of the tensor branch moduli space can be quite different from the $\mathcal{N}=(2,0)$ case, including the possibility of forbidden zones. In compactifications to two dimensions, studying such walls suggests the presence of additional light states and / or strong coupling effects. It would be most instructive to understand this issue in explicit examples.

We have also studied some aspects of discrete quotients upon compactification (as well as sometimes in six dimensions!). In the comparatively simpler setting of compactifications of class $\mathcal{S}$ theories on a Riemann surface, "discrete twists," namely adding background chemical potentials for flavor symmetries along one-cycles leads to a broad class of $\mathcal{N}=2$ theories. There is also an important interplay between punctures [4] and twists [81, 82, 116]. Extending this to the case of punctures for $(1,0)$ theories such as the class $\mathcal{S}_{\Gamma}$ theories (see e.g. $[45,62,64])$ would seem worthwhile to investigate further.

\section{Acknowledgments}

We thank M. Del Zotto, T.T. Dumitrescu, A. Grassi, K. Intriligator, N. Mekareeya, D.R. Morrison, D.S. Park, L. Tizzano, A. Tomasiello, A. Trimm, and K. Yonekura for helpful discussions. We also thank M. Del Zotto, D.R. Morrison, A. Tomasiello, and A. Trimm for helpful comments on an earlier draft. JJH thanks the Aspen Center for Physics Winter Conference on Superconformal Field Theories in $d \geq 4$, NSF grant PHY-1066293, for hospitality during part of this work. The work of FA and JJH is supported by NSF CAREER grant PHY-1452037. FA also acknowledges support from NSF grant PHY1620311. The work of TR is supported by NSF grant PHY-1067976 and by the NSF GRF under DGE-1144152. 


\section{A Anomaly polynomial and Green-Schwarz redundancy}

In this appendix we discuss some further details on the structure of the anomaly polynomial, and redundancy in the Green-Schwarz terms. The main notion we wish to explore here is the additional structure which results when the number of simple gauge groups is the same as the number of tensor multiplets. In fact, we will aim to show how a general form for the anomaly polynomial can be obtained by "analytic continuation" in the ranks of the gauge groups. Along these lines, we shall find it convenient to label the Green-Schwarz terms as:

$$
L_{6 D} \supset \int \mu_{i}^{j} B^{(i)} \wedge \operatorname{Tr}\left(F_{(j)} \wedge F_{(j)}\right),
$$

where now $\mu$ is a square matrix, and we only include couplings to dynamical gauge fields, i.e. we drop all couplings to background flavor symmetry field strengths. In this special case, we observe that the $\mu$ 's are square matrices. Moreover, due to the placement of indices, they are related to our previous presentation of couplings as:

$$
\mu_{i}^{k}=\mu_{i, j}\left(A^{-1}\right)^{j k} .
$$

In the fundamental chamber where $\mu_{i, j}=A_{i j}$, we have $\mu_{i}^{k}=\delta_{i}^{k}$, the identity matrix. Indeed, since we can label the chambers of moduli space by elements of the automorphism group, we see that the $\mu$ 's can all be identified with group elements of Aut $(\Lambda)$. Note also that in this basis, both $\mu$ and $\mu^{-1}$ will be integral matrices.

Our plan in this appendix will be to first review some elements of the anomaly polynomial in general terms, and then explain how to compute the $\mu$ 's in this case via analytic continuation in the ranks of gauge groups.

The anomaly polynomial eight-form of the theory splits into a 1-loop piece and a GreenSchwarz piece. At the 1-loop level, there is a contribution from the tensor multiplets, the vector multiplets, and the hypermultiplets. Each tensor multiplet contributes

$$
I_{\mathrm{tens}}=\frac{1}{24}\left(c_{2}(R)^{2}+\frac{1}{2} c_{2}(R) p_{1}(T)+\frac{1}{240}\left(23 p_{1}(T)^{2}-116 p_{2}(T)\right)\right),
$$

where $R$ denotes the SU(2) R-symmetry bundle and $T$ the formal tangent bundle. From a gauge group $G_{i}$ with field strength $F_{i}$, the vector multiplets contribute

$$
\begin{aligned}
I_{\mathrm{vec}}= & -\frac{\operatorname{tr}\left(F_{i}^{4}\right)+6 c_{2} \operatorname{tr}\left(F_{i}^{2}\right)+d_{G_{i}} c_{2}(R)^{2}}{24}-p_{1}(T)\left(\frac{\operatorname{tr}\left(F_{i}^{2}\right)+d_{G_{i}} c_{2}(R)}{48}\right) \\
& -d_{G_{i}}\left(\frac{7 p_{1}(T)^{2}-4 p_{2}(T)}{5760}\right),
\end{aligned}
$$

where the trace, $\operatorname{tr}$ is taken over the adjoint representation of the gauge group $G_{i}$. Finally, a hypermultiplet in representation $\tilde{\rho}_{i}$ of a symmetry with field strength $F_{i}$ contributes

$$
I_{\text {hyp }}=\tilde{r}_{i} \frac{\left(7 p_{1}(T)^{2}-4 p_{2}(T)\right)}{5760}+\frac{\operatorname{tr}_{\tilde{\rho}_{i}}\left(F_{i}^{2}\right) p_{1}(T)}{48}+\frac{\operatorname{tr}_{\tilde{\rho}_{i}}\left(F_{i}^{4}\right)}{24},
$$

where $\tilde{r}_{i}$ is the dimension of the representation $\tilde{\rho}_{i}$. Sometimes we can have also halfhypermultiplets. This will mean that the associated anomaly polynomial contribution 
is divided by a factor of 2 . There can also be hypermultiplets in mixed representations $\rho_{i}, \rho_{i+1}$, which contribute

$$
\begin{aligned}
I_{\text {hyp }- \text { mix }}= & \frac{1}{5760} r_{i} r_{i+1}\left(7 p_{1}(T)^{2}-4 p_{2}(T)\right)+\frac{1}{48} p_{1}(T)\left(r_{i} \operatorname{tr}_{\rho_{i+1}}\left(F_{i+1}^{2}\right)+r_{i+1} \operatorname{tr}_{\rho_{i}}\left(F_{i}^{2}\right)\right) \\
& +\frac{1}{24}\left(r_{i} \operatorname{tr}_{\rho_{i+1}}\left(F_{i+1}^{4}\right)+r_{i+1} \operatorname{tr}_{\rho_{i}}\left(F_{i}^{4}\right)+6 \operatorname{tr}_{\rho_{i+1}}\left(F_{i+1}^{2}\right) \operatorname{tr}_{\rho_{i}}\left(F_{i}^{2}\right)\right),
\end{aligned}
$$

where the trace is taken over the representations $\rho_{i}$, which is usually the fundamental, and $r_{i}$ are the dimension of the representations $\rho_{i}$. The same formula applies if one of the symmetry groups $G_{i}$ is a global symmetry, with the associated $F_{i}$ now a background field strength. In IIB language, these flavor symmetries arise from 7-branes wrapping a non-compact component of the discriminant locus.

Following the discussion in appendix A of reference [39], we can express the traces of the gauge field strength monomials on some representation as follows

$$
\operatorname{tr}_{\rho_{i}}\left(F_{i}^{4}\right)=\alpha_{\rho_{i}} \operatorname{Tr}\left(F_{i}^{4}\right)+\frac{3}{4} c_{\rho_{i}}\left(\operatorname{Tr}\left(F_{i}^{2}\right)\right)^{2} \quad \operatorname{tr}_{\rho_{i}}\left(F_{i}^{2}\right)=\operatorname{Ind}\left(\rho_{i}\right) \operatorname{Tr}\left(F_{i}^{2}\right)
$$

where Ind is the index of the representation. For the adjoint and the fundamental, these equations become

$$
\begin{aligned}
\operatorname{tr}\left(F_{i}^{4}\right) & =t_{G_{i}} \operatorname{Tr}\left(F_{i}^{4}\right)+\frac{3}{4} u_{G_{i}}\left(\operatorname{Tr}\left(F_{i}^{2}\right)\right)^{2} & \operatorname{tr}\left(F_{i}^{2}\right) & =h_{G_{i}}^{\vee} \operatorname{Tr}\left(F_{i}^{2}\right) \\
\operatorname{tr}_{\text {fund }}\left(F_{i}^{4}\right) & =\operatorname{Tr}\left(F_{i}^{4}\right) & \operatorname{tr}_{\text {fund }}\left(F_{i}^{2}\right) & =s_{G_{i}} \operatorname{Tr}\left(F_{i}^{2}\right),
\end{aligned}
$$

where $\left\{t_{G_{i}}, u_{G_{i}}, h_{G_{i}}^{\vee}, s_{G_{i}}\right\}$ are the group theory data defined in appendix A of [41]. In particular, $h_{G_{i}}^{\vee}$ is the dual Coxeter number of the group $G_{i}$. For gauge groups $G_{i}$ without an independent quartic Casimir, we have $\alpha_{\rho_{i}}=0$ for every representation $\rho_{i}$, and $\operatorname{tr}_{\text {fund }}\left(F_{i}^{4}\right)=$ $\frac{3}{4} c_{\text {fund }}\left(\operatorname{Tr}\left(F_{i}^{2}\right)\right)^{2}$.

The one-loop contribution of the anomaly polynomial is given by the sum of all these terms

$$
I^{1-\text { loop }}=I_{\text {tens }}+I_{\mathrm{vec}}+I_{\mathrm{hyp}}+I_{\mathrm{hyp}-\mathrm{mix}}
$$

This differs from the prescription of [41] slightly in that we are not including contributions from empty -1 curves that are not paired with gauge groups, also known as "E-strings." In the following section, we will show that these E-string contributions can be treated via an analytic continuation, so there is no need to place them on a different footing from the rest of the contributions.

In order for the theory to be consistent, we need to cancel all the terms in $I^{1-\text { loop }}$ that involve field strengths of gauge groups. This is done by a Green-Schwarz mechanism. For this, we introduce the intersection pairing on the curves $\Sigma_{i}$ in $B_{2}$,

$$
A_{i j}=-\Sigma_{i} \cap \Sigma_{j}
$$

In what follows, we assume that the number of gauge groups is the same as the number of tensor multiplets. This allows us to conflate gauge group indices with tensor indices, and it also means that the matrix $\mu$ will be square (and in fact invertible). In the following 
subsection, we will see that by a suitable analytic continuation to $\mathfrak{s p}(0)$ or $\mathfrak{s u}(1)$, we can think of all tensor multiplets as being paired with a gauge group, justifying this assumption.

The Green-Schwarz term contribution to the anomaly eight-form reads:

$$
I_{\mathrm{GS}}=\frac{1}{2} A_{i j} I^{i} I^{j}, \quad i, j=1, \ldots, N_{T},
$$

where the $I^{i}$ are defined as follows:

$$
I^{i}=\left(\sum_{j=1}^{N_{T}}\left(\mu^{-1}\right)_{j}^{i} \frac{\operatorname{Tr}\left(F_{j}^{2}\right)}{4}+y_{i} c_{2}(R)+K_{i} \frac{p_{1}(T)}{12}+z_{i} \frac{\operatorname{Tr}\left(F_{(f l) i}^{2}\right)}{4}\right) .
$$

By charge quantization, the matrix $\mu^{-1}$ must be integral. Gauge anomaly cancellation requires that the combination $I^{1-\mathrm{loop}}+I_{\mathrm{GS}}$ should be independent of any gauge field strengths $\operatorname{Tr}\left(F_{j}^{2}\right)$. Note that the coefficient in front of $\operatorname{Tr}\left(F_{i}^{4}\right)$ cannot be canceled by a GS type mechanism, so this must vanish at 1-loop. In the final analysis, the anomaly cancellation conditions for each gauge group $G_{i}$ become [117]:

$$
\begin{aligned}
t_{G_{i}}-\sum_{\rho_{i}} \alpha_{\rho_{i}} n_{\rho_{i}} & =0 \\
u_{G_{i}}-\sum_{\rho_{i}} c_{\rho_{i}} n_{\rho_{i}} & =A_{i i} \\
h_{G_{i}}^{\vee}-\sum_{\rho_{i}} \operatorname{Ind}\left(\rho_{i}\right) n_{\rho_{i}} & =-6+3 A_{i i} \\
\sum_{\rho_{i}, \rho_{j}} \operatorname{Ind}\left(\rho_{i}\right) \operatorname{Ind}\left(\rho_{j}\right) n_{\rho_{i}, \rho_{j}} & =-\frac{1}{4} A_{i j} .
\end{aligned}
$$

Here, $n_{\rho_{i}}$ is the number of hypermultiplets in the $\rho_{i}$ th representation of the gauge group $G_{i}$, and $n_{\rho_{i}, \rho_{j}}$ is the number of hypermultiplets in the mixed representation $\left(\rho_{i}, \rho_{j}\right)$ of $G_{i} \times G_{j}$. Note that some of the hypermultiplets in the sums in (A.14a)-(A.14c) might also be charged under an additional flavor or symmetry group i.e. they might be in mixed representations.

\section{A.1 All Green-Schwarz terms via analytic continuation}

For -1 or -2 curves without gauge algebras used as matter in a 6D SCFT, one can compute the anomaly polynomial by analytically continuing $\operatorname{Sp}(k)$ to $k=0$ and $\mathrm{SU}(k)$ to $k=1$, respectively. Indeed, through this analytic continuation, we may compute the GS terms in any phase of any 6D SCFT. We begin with the SCFT quiver as well as the matrix $\mu$ defined in (2.3)

Next, for a given curve $\Sigma_{i}$ with $\Sigma_{i} \cap \Sigma_{i}=-n$ carrying gauge group $G_{i}$ with field strength $F_{i}$, define $I_{i}^{(0)}$ by the following:

$$
I_{i}^{(0)}=h_{G}^{\vee} c_{2}(R)+\frac{(n-2)}{4} p_{1}(T)+\frac{n}{4} \operatorname{Tr} F_{i}^{2}-\sum_{j \in \mathrm{nn}} \frac{1}{4} \operatorname{Tr} F_{j}^{2} .
$$

Here, $h_{G}^{\vee}$ is the dual coxeter number of $G$, and the sum runs over "nn," the "nearest neighbors" of the curve $\Sigma_{i}$, which are simply the curves $\Sigma_{j}$ that intersect it at a point. Note that the coefficient of the gauge field strength $\operatorname{Tr} F_{j}^{2}$ in $I_{i}^{(0)}$ is given simply by $\frac{1}{4} A_{i j}$. 
For an empty -1 curve, also known as the rank 1 E-string theory, we use the analytic continuation of $\operatorname{Sp}(k)$ to $k=0$ and set $F_{i}=0$, yielding

$$
I_{\mathrm{E}-\text { string }}^{(0)}=c_{2}(R)-\frac{1}{4} p_{1}(T)-\sum_{j \in \mathrm{nn}} \frac{1}{4} \operatorname{Tr} F_{j}^{2} .
$$

For an empty -2 curve, corresponding to the $A_{1} 6 \mathrm{D}$ SCFT we use the analytic continuation from $\mathrm{SU}(k)$ to $\mathrm{SU}(1)$, set $F_{i}=0$, and associate a global $\mathrm{SU}(2)_{L}$ symmetry with the curve. This last stipulation amounts to replacing $\sum_{j \in \mathrm{nn}} \frac{1}{4} \operatorname{Tr} F_{j}^{2}$ with $c_{2}(L)$,

$$
I_{A_{1}}^{(0)}=c_{2}(R)-c_{2}(L)
$$

So far, the vectors $I_{i}^{(0)}$ specify the anomalies for the trivial phase of the geometry, with $\mu=\sigma=I d$. If we now transform to a different phase by an automorphism $\sigma$, we find a new matrix $\mu=\sigma$. In this phase, we define the Green-Schwarz vectors $I_{i}$ by

$$
I_{i}=\sum_{j} \mu_{i}^{j} I_{j}^{(0)} .
$$

The Green-Schwarz contribution to the anomaly polynomial is then given by

$$
I_{\mathrm{GS}}=\frac{1}{2}\left(A^{-1}\right)^{i j} I_{i} I_{j}
$$

The full anomaly polynomial is then given by a sum of $I_{\mathrm{GS}}$ and $I^{1-\text { loop }}$, which is computed via (A.10). For a paired tensor, the rules given in appendix A of [41] may be used to compute $I^{1-\text { loop }}$. For an unpaired -1 tensor, we simply get a contribution from the tensor multiplet, as shown in (A.3). For an unpaired -2 tensor, we add the contribution from the tensor multiplet as well as the contribution from a single free hypermultiplet charged as a half-doublet under the $c_{2}(L)$ symmetry,

$$
I_{\text {free }}=\frac{1}{24} c_{2}(L)^{2}+\frac{1}{48} c_{2}(L) p_{1}(T)+\frac{7 p_{1}(T)^{2}-4 p_{2}(T)}{5760} .
$$

If the unpaired -2 tensor is adjacent to a tensor carrying $\mathrm{SU}(2)_{G}$ gauge symmetry, the $\mathrm{SU}(2)_{L}$ is gauged, and we replace $c_{2}(L)$ in (A.17) and (A.20) with $\frac{1}{4} \operatorname{Tr}\left(F_{\mathrm{SU}(2)_{G}}\right)^{2}$.

One might wonder whether this analytic continuation truly gives the full set of allowed Green-Schwarz couplings. When the number of tensor multiplets is equal to the number of gauge groups (i.e. there are no unpaired tensors), one verifies using line (A.13) that each choice of $\mu$ gives rise to a unique choice for the Green-Schwarz couplings.

In physical theories where there are unpaired tensors, the matrix of couplings for the $\mu$ 's are no longer square. By analytic continuation on the groups, however, we can always extend this to a square matrix. This analytic always appears to yield a unique answer. Indeed, the perspective of F-theory compactification, we note that the geometric phases of the base are completely characterized by the automorphism group of the lattice. This means that the choices of $\mu$ for a theory with -1 or -2 curves should be independent of whether these curves are paired with gauge groups or not. This in turn fixes the Green-Schwarz 
couplings $I_{i}$ for unpaired tensors to the values specified by the analytic continuations in (A.16) and (A.17). Thus, we expect from F-theory that these analytic continuations give the unique Green-Schwarz couplings.

In [86], the possibility of "outlier" 6D SCFTs that do not admit known F-theory constructions was discussed, and several such theories were proposed. In particular, one may consider a theory with base

$$
4|| 2,2,2, \ldots, 2
$$

where $\|$ indicates that the -4 curve is tangent to the adjacent -2 curve. In terms of the Dirac pairing, such a tangency implies $A_{12}=A_{21}=-2$. All of the anomaly cancellation conditions are satisfied for this theory, and (A.15) is modified straightforwardly by adding a factor of 2 in the sum over nearest neighbors for the tangency, so that the coefficient of the gauge field strength $\operatorname{Tr} F_{j}^{2}$ in $I_{i}^{(0)}$ is still given by $\frac{1}{4} A_{i j}$.

However, one may also consider an outlier theory with $\mathrm{SU}(N)$ gauge group for $N \geq 8$ on a -1 tensor with $N_{F}=N-8$ fundamental hypermultiplets and a symmetric hypermultiplet. This theory violates (A.14c) and therefore has non-vanishing gauge-gravitational anomalies at 1-loop, but a suitable Green-Schwarz term can cancel this term. In particular, all gauge anomalies will cancel if we take

$$
I_{1}^{(0)}=N c_{2}(R)+\frac{1}{4} p_{1}(T)+\frac{1}{4} \operatorname{Tr} F_{\mathrm{SU}(N)}^{2}-\frac{1}{4} \operatorname{Tr} F_{N_{F}}^{2} .
$$

Note that the coefficient of $p_{1}(T)$ is $+1 / 4$ rather than the $-1 / 4$ expected from (A.15). It appears that the above analytic continuation does not apply to such "outlier" theories. It would be interesting to see whether there is a further refinement in this analysis by interpreting these outlier theories in terms of a discrete quotient, along the lines presented in this paper.

In the following subsection, we will show how the above formulae work in a handful of simple examples.

\section{A.2 Examples}

\section{A.2.1 Rank $Q$ E-string theory}

Let us consider the rank $Q$ E-string theory. The anomaly polynomial of this theory was computed in [39]:

$$
I_{\mathrm{rankQ}}^{\mathrm{E}-\mathrm{string}}=\frac{Q^{2}}{6}\left(c_{2}(L)-c_{2}(R)\right)^{2}+\frac{Q}{2}\left(c_{2}(L)-c_{2}(R)\right) I_{4}^{2}+Q\left(\frac{1}{4} I_{4}-I_{8}\right) .
$$

The contributions $I_{4}$ and $I_{8}$ are given by

$$
\begin{aligned}
& I_{4}=\frac{1}{4}\left(\operatorname{Tr}\left(F_{E_{8}}^{2}\right)+p_{1}(T)-2 c_{2}(L)-2 c_{2}(R)\right), \\
& I_{8}=\frac{1}{48}\left(c_{2}(L)-c_{2}(R)\right)^{2}+p_{2}(T)-\frac{1}{4}\left(2 c_{2}(L)+2 c_{2}(R)+p_{1}(T)\right),
\end{aligned}
$$

where $F_{E_{8}}$ is the field strength of the global $E_{8}$ symmetry for the theory. 
We begin with the special case $Q=1$, namely the rank 1 E-string theory. This can be viewed as the theory of an M5-brane on the M9-wall of Heterotic M-theory. Here, $c_{2}(L)$ is the second Chern class of the $\mathrm{SU}(2)_{L}$ bundle associated to the transverse space of the M5 brane, which together with the $\mathrm{SU}(2)_{R}$ makes up the normal bundle associated to the $\mathrm{SO}(4)$ global symmetry of the theory. The -1 curve usually appears as generalized matter in the $6 \mathrm{D}(1,0)$ theories, and as in [41], we need to subtract the free hypermultiplet contribution given by

$$
I_{\text {free-hyp }}=\frac{1}{24}\left(c_{2}(L)^{2}+\frac{1}{2} c_{2}(L) p_{1}(T)+\frac{1}{240}\left(7 p_{1}(T)^{2}-4 p_{2}(T)\right)\right),
$$

Using our formulae from above, we have

$$
I_{\mathrm{E}-\text { string }}^{i}=c_{2}(R)-\frac{1}{4} p_{1}(T)-\frac{1}{4} \operatorname{Tr}\left(F_{E_{8}}^{2}\right) .
$$

This gives

$$
\begin{aligned}
I_{\mathrm{GS}}= & \frac{1}{2}\left(I_{\mathrm{E}-\text { string }}^{i}\right)^{2} \\
= & \frac{1}{2}\left[c_{2}(R)^{2}-\frac{1}{2} c_{2}(R) p_{1}(T)-\frac{1}{2} c_{2}(R) \operatorname{Tr}\left(F_{E_{8}}^{2}\right)+\frac{1}{16} p_{1}(T)^{2}\right. \\
& \left.\quad+\frac{1}{8} p_{1}(T) \operatorname{Tr}\left(F_{i-1}^{2}\right)+\frac{1}{8} p_{1}(T) \operatorname{Tr}\left(F_{i+1}^{2}\right)+\frac{1}{16} \operatorname{Tr}\left(F_{E_{8}}^{2}\right)^{2}\right] .
\end{aligned}
$$

To this, we add the 1-loop piece associated with a tensor multiplet to get

$$
\begin{aligned}
I_{\text {tot }}= & \frac{13}{24} c_{2}(R)^{2}-\frac{11}{48} c_{2}(R) p_{1}(T)+\frac{203}{5760} p_{1}(T)^{2}-\frac{29}{1440} p_{2}(T)-\frac{1}{4} c_{2}(R) \operatorname{Tr}\left(F_{E_{8}}^{2}\right) \\
& +\frac{1}{16} p_{1}(T) \operatorname{Tr}\left(F_{E_{8}}^{2}\right)+\frac{1}{32} \operatorname{Tr}\left(F_{E_{8}}^{2}\right)^{2}
\end{aligned}
$$

This is precisely the anomaly polynomial of the rank 1 E-string with $I_{\text {free-hyp }}$ subtracted off. Next, we consider the rank $Q$ E-string. We have

$$
A_{i j}=\left[\begin{array}{cccc}
1 & -1 & 0 & \ldots \\
-1 & 2 & -1 & \ldots \\
\vdots & & & \vdots \\
\ldots & 0 & -1 & 2
\end{array}\right]
$$

Using (A.16) and (A.17), we have

$$
\begin{aligned}
& I_{1}=c_{2}(R)-\frac{1}{4} p_{1}(T)-\frac{1}{4} \operatorname{Tr}\left(F_{E_{8}}\right)^{2} \\
& I_{k}=c_{2}(R)-c_{2}(L), \quad k=2, \ldots, Q .
\end{aligned}
$$

The Green-Schwarz term is then given by $I_{\mathrm{GS}}=\frac{1}{2} I_{i}\left(A^{-1}\right)^{i j} I_{j}$. To this, we add the 1-loop contribution associated with $Q$ tensor multiplets (obtained by taking $N_{T}=\mathrm{Q}$ copies of 
line (A.3)) and $Q-1$ free hypermultiplets $\left(Q-1\right.$ times $I_{\text {free }}$ of (A.20)). We arrive at

$$
\begin{aligned}
I_{\text {tot }}= & -\frac{1}{24} c_{2}(L)^{2}-\frac{1}{48} c_{2}(L) p_{1}(T)-\frac{7}{5760} p_{1}(T)^{2}+\frac{1}{1440} p_{2}(T)+\frac{Q}{8} c_{2}(L)^{2} \\
& +\frac{Q}{3} c_{2}(L) c_{2}(R)+\frac{Q}{8} c_{2}(R)^{2}-\frac{5 Q}{48} c_{2}(L) p_{1}(T)-\frac{5 Q}{48} c_{2}(R) p_{1}(T)+\frac{7 Q}{192} p_{1}(T)^{2} \\
& -\frac{Q}{48} p_{2}(T)-\frac{Q^{2}}{4} c_{2}(L)^{2}+\frac{Q^{2}}{4} c_{2}(R)^{2}+\frac{Q^{2}}{8} c_{2}(L) p_{1}(T)-\frac{Q^{2}}{8} c_{2}(R) p_{1}(T) \\
& +\frac{Q^{3}}{6} c_{2}(L)^{2}-\frac{Q^{3}}{3} c_{2}(L) c_{2}(R)+\frac{Q^{3}}{6} c_{2}(R)^{2}-\frac{Q}{8} c_{2}(L) \operatorname{Tr}\left(F_{E_{8}}^{2}\right)-\frac{Q}{8} c_{2}(R) \operatorname{Tr}\left(F_{E_{8}}^{2}\right) \\
& +\frac{Q}{16} p_{1}(T) \operatorname{Tr}\left(F_{E_{8}}^{2}\right)+\frac{Q^{2}}{8} c_{2}(L) \operatorname{Tr}\left(F_{E_{8}}^{2}\right)-\frac{Q^{2}}{8} c_{2}(R) \operatorname{Tr}\left(F_{E_{8}}^{2}\right)+\frac{Q}{32} \operatorname{Tr}\left(F_{E_{8}}^{2}\right)^{2} .
\end{aligned}
$$

This is precisely (A.26) with the contribution of a free hypermultiplet subtracted off.

\section{A.2.2 SO(10)-Sp(1)}

We next consider the $\mathrm{SO}(10)-\mathrm{Sp}(1)$ gauge theory with Dirac pairing:

$$
A_{i j}=\left[\begin{array}{cc}
4 & -1 \\
-1 & 1
\end{array}\right] \text {. }
$$

We know that there are four phases, given by:

$$
\mu=\sigma= \pm I, \quad \mu=\sigma= \pm\left[\begin{array}{cc}
1 & 2 \\
0 & -1
\end{array}\right]
$$

For simplicity, we consider only the phase where both $t_{1}, t_{2}>0$. For $\mu=I$, we have $\left(\mu^{-1}\right)^{T}=I$, so $I_{1}=I_{1}^{(0)}, I_{2}=I_{2}^{(0)}$. Using (A.15),

$$
\begin{aligned}
& I_{1}=8 c_{2}(R)+\frac{1}{2} p_{1}(T)+\operatorname{Tr} F_{1}^{2}-\frac{1}{4} \operatorname{Tr} F_{L}^{2}-\frac{1}{4} \operatorname{Tr} F_{2}^{2}, \\
& I_{2}=2 c_{2}(R)-\frac{1}{4} p_{1}(T)+\frac{1}{4} \operatorname{Tr} F_{2}^{2}-\frac{1}{4} \operatorname{Tr} F_{R}^{2}-\frac{1}{4} \operatorname{Tr} F_{1}^{2} .
\end{aligned}
$$

It is easily checked that this produces the correct Green-Schwarz term $I_{\mathrm{GS}}=\frac{1}{2} A^{i j} I_{i} I_{j}$.

The other solution is just slightly more complicated. We have

$$
\mu=\left[\begin{array}{cc}
1 & 2 \\
0 & -1
\end{array}\right] \text {. }
$$

Eq. (A.18) yields

$$
\begin{aligned}
& I_{1}=12 c_{2}(R)+\frac{1}{2} \operatorname{Tr} F_{1}^{2}+\frac{1}{4} \operatorname{Tr} F_{2}^{2}-\frac{1}{4} \operatorname{Tr} F_{L}^{2}-\frac{1}{2} \operatorname{Tr} F_{R}^{2}, \\
& I_{2}=-2 c_{2}(R)+\frac{1}{4} \operatorname{Tr} F_{1}^{2}-\frac{1}{4} \operatorname{Tr} F_{2}^{2}+\frac{1}{4} \operatorname{Tr} F_{R}^{2}+\frac{1}{4} p_{1}(T)^{2} .
\end{aligned}
$$

It can also be checked that this reproduces the correct GS term $I_{\mathrm{GS}}$. Raising indices with the metric, we find

$$
\begin{aligned}
& I^{1} \supset \frac{1}{4}\left(\operatorname{Tr} F_{1}^{2}\right) \\
& I^{2} \supset \frac{1}{4}\left(2 \operatorname{Tr} F_{1}^{2}-\operatorname{Tr} F_{2}^{2}\right) .
\end{aligned}
$$


This agrees with charge quantization. Note also that

$$
\left[\begin{array}{l}
I^{1} \\
I^{2}
\end{array}\right] \supset \frac{1}{4}\left[\begin{array}{cc}
1 & 0 \\
2 & -1
\end{array}\right]\left[\begin{array}{c}
\operatorname{Tr} F_{1}^{2} \\
\operatorname{Tr} F_{2}^{2}
\end{array}\right]=\frac{1}{4}\left(\mu^{-1}\right)^{T}\left[\begin{array}{c}
\operatorname{Tr} F_{1}^{2} \\
\operatorname{Tr} F_{2}^{2}
\end{array}\right],
$$

in accord with line (A.13).

\section{A.2.3 $\mathrm{SU}\left(N_{1}\right)-\mathrm{SU}\left(N_{2}\right)$}

We consider now a quiver consisting of two -2 curves carrying gauge groups $\mathrm{SU}\left(N_{1}\right)$ and $\mathrm{SU}\left(N_{2}\right)$, respectively. In this case, we have:

$$
A_{i j}=\left[\begin{array}{cc}
2 & -1 \\
-1 & 2
\end{array}\right]
$$

and

$$
\begin{aligned}
I_{1}^{(0)} & =N_{1} c_{2}(R)+\frac{1}{2} \operatorname{Tr} F_{1}^{2}-\frac{1}{4} \operatorname{Tr} F_{L}^{2}-\frac{1}{4} \operatorname{Tr} F_{2}^{2}, \\
I_{2}^{(0)} & =N_{2} c_{2}(R)+\frac{1}{2} \operatorname{Tr} F_{2}^{2}-\frac{1}{4} \operatorname{Tr} F_{R}^{2}-\frac{1}{4} \operatorname{Tr} F_{1}^{2} .
\end{aligned}
$$

There are a number of different phases. We will consider simply the one with

$$
\mu=\left[\begin{array}{cc}
0 & 1 \\
-1 & -1
\end{array}\right] \text {. }
$$

So,

$$
\begin{aligned}
& I_{1}=N_{2} c_{2}(R)+\frac{1}{2} \operatorname{Tr} F_{2}^{2}-\frac{1}{4} \operatorname{Tr} F_{R}^{2}-\frac{1}{4} \operatorname{Tr} F_{1}^{2}, \\
& I_{2}=-\left(N_{1}+N_{2}\right) c_{2}(R)-\frac{1}{4} \operatorname{Tr} F_{1}^{2}-\frac{1}{4} \operatorname{Tr} F_{2}^{2}+\frac{1}{4} \operatorname{Tr} F_{L}^{2}+\frac{1}{4} \operatorname{Tr} F_{R}^{2} .
\end{aligned}
$$

This gives the correct GS term, $\frac{1}{2}\left(A^{-1}\right)^{i j} I_{i} I_{j}$, and we have

$$
\begin{aligned}
& I^{1} \supset \frac{1}{4}\left(-\operatorname{Tr} F_{1}^{2}+\operatorname{Tr} F_{2}^{2}\right) \\
& I^{2} \supset \frac{1}{4}\left(-\operatorname{Tr} F_{1}^{2}\right) .
\end{aligned}
$$

This agrees with charge quantization, and

$$
\left[\begin{array}{l}
I^{1} \\
I^{2}
\end{array}\right] \supset \frac{1}{4}\left[\begin{array}{ll}
-1 & 1 \\
-1 & 0
\end{array}\right]\left[\begin{array}{c}
\operatorname{Tr} F_{1}^{2} \\
\operatorname{Tr} F_{2}^{2}
\end{array}\right]=\frac{1}{4}\left(\mu^{-1}\right)^{T}\left[\begin{array}{c}
\operatorname{Tr} F_{1}^{2} \\
\operatorname{Tr} F_{2}^{2}
\end{array}\right]
$$

\section{A.2.4 Sp(1)-SO(10)-Sp(1)}

Finally, we consider a quiver with three simple gauge group factors, namely $\mathrm{Sp}(1) \times \mathrm{SO}(10) \times$ $\mathrm{Sp}(1)$. We have

$$
A_{i j}=\left[\begin{array}{ccc}
1 & -1 & 0 \\
-1 & 4 & -1 \\
0 & -1 & 1
\end{array}\right]
$$


and

$$
\begin{aligned}
& I_{1}^{(0)}=2 c_{2}(R)-\frac{1}{4} p_{1}(T)+\frac{1}{4} \operatorname{Tr} F_{1}^{2}-\frac{1}{4} \operatorname{Tr} F_{L}^{2}-\frac{1}{4} \operatorname{Tr} F_{2}^{2}, \\
& I_{2}^{(0)}=8 c_{2}(R)+\frac{1}{2} p_{1}(T)+\operatorname{Tr} F_{2}^{2}-\frac{1}{4} \operatorname{Tr} F_{3}^{2}-\frac{1}{4} \operatorname{Tr} F_{1}^{2}, \\
& I_{3}^{(0)}=2 c_{2}(R)-\frac{1}{4} p_{1}(T)+\frac{1}{4} \operatorname{Tr} F_{3}^{2}-\frac{1}{4} \operatorname{Tr} F_{R}^{2}-\frac{1}{4} \operatorname{Tr} F_{2}^{2} .
\end{aligned}
$$

There are many choices of $\mu$ that cancel gauge anomalies and satisfy charge quantization. One choice is

$$
\mu=\left[\begin{array}{ccc}
-1 & 0 & 0 \\
0 & -1 & -2 \\
0 & 0 & 1
\end{array}\right]
$$

Using (A.18), we have

$$
\begin{aligned}
& I_{1}=-2 c_{2}(R)+\frac{1}{4} p_{1}(T)-\frac{1}{4} \operatorname{Tr} F_{1}^{2}+\frac{1}{4} \operatorname{Tr} F_{L}^{2}+\frac{1}{4} \operatorname{Tr} F_{2}^{2}, \\
& I_{2}=-12 c_{2}(R)-\frac{1}{2} \operatorname{Tr} F_{2}^{2}-\frac{1}{4} \operatorname{Tr} F_{3}^{2}+\frac{1}{4} \operatorname{Tr} F_{1}^{2}+\frac{1}{2} \operatorname{Tr} F_{R}^{2}, \\
& I_{3}=2 c_{2}(R)-\frac{1}{4} p_{1}(T)+\frac{1}{4} \operatorname{Tr} F_{3}^{2}-\frac{1}{4} \operatorname{Tr} F_{R}^{2}-\frac{1}{4} \operatorname{Tr} F_{2}^{2} .
\end{aligned}
$$

This gives the correct $I_{\mathrm{GS}}$ and satisfies

$$
\left[\begin{array}{l}
I^{1} \\
I^{2} \\
I^{3}
\end{array}\right] \supset \frac{1}{4}\left[\begin{array}{ccc}
-1 & 0 & 0 \\
0 & -1 & 0 \\
0 & -2 & 1
\end{array}\right]\left[\begin{array}{c}
\operatorname{Tr} F_{1}^{2} \\
\operatorname{Tr} F_{2}^{2} \\
\operatorname{Tr} F_{3}^{2}
\end{array}\right]=\frac{1}{4}\left(\mu^{-1}\right)^{T}\left[\begin{array}{c}
\operatorname{Tr} F_{1}^{2} \\
\operatorname{Tr} F_{2}^{2} \\
\operatorname{Tr} F_{3}^{2}
\end{array}\right] .
$$

Open Access. This article is distributed under the terms of the Creative Commons Attribution License (CC-BY 4.0), which permits any use, distribution and reproduction in any medium, provided the original author(s) and source are credited.

\section{References}

[1] C. Vafa, Geometric origin of Montonen-Olive duality, Adv. Theor. Math. Phys. 1 (1998) 158 [hep-th/9707131] [INSPIRE].

[2] E. Witten, Solutions of four-dimensional field theories via M-theory, Nucl. Phys. B 500 (1997) 3 [hep-th/9703166] [INSPIRE].

[3] P.C. Argyres and N. Seiberg, S-duality in $N=2$ supersymmetric gauge theories, JHEP 12 (2007) 088 [arXiv:0711.0054] [INSPIRE].

[4] D. Gaiotto, $N=2$ dualities, JHEP 08 (2012) 034 [arXiv:0904.2715] [INSPIRE].

[5] J.J. Heckman, D.R. Morrison and C. Vafa, On the classification of 6D SCFTs and generalized ADE orbifolds, JHEP 05 (2014) 028 [Erratum ibid. 06 (2015) 017] [arXiv:1312.5746] [INSPIRE].

[6] M. Del Zotto, J.J. Heckman, A. Tomasiello and C. Vafa, 6D conformal matter, JHEP 02 (2015) 054 [arXiv: 1407.6359] [inSPIRE]. 
[7] J.J. Heckman, D.R. Morrison, T. Rudelius and C. Vafa, Atomic classification of 6D SCFTs, Fortsch. Phys. 63 (2015) 468 [arXiv:1502.05405] [inSPIRE].

[8] F. Apruzzi, M. Fazzi, D. Rosa and A. Tomasiello, All AdS $S_{7}$ solutions of type-II supergravity, JHEP 04 (2014) 064 [arXiv: 1309.2949] [InSPIRE].

[9] D. Gaiotto and A. Tomasiello, Holography for (1,0) theories in six dimensions, JHEP 12 (2014) 003 [arXiv: 1404.0711] [InSPIRE].

[10] L. Bhardwaj, Classification of $6 d \mathcal{N}=(1,0)$ gauge theories, JHEP 11 (2015) 002 [arXiv: 1502 .06594] [INSPIRE].

[11] E. Witten, String theory dynamics in various dimensions, Nucl. Phys. B 443 (1995) 85 [hep-th/9503124] [INSPIRE].

[12] E. Witten, Some comments on string dynamics, in the proceedings of Strings'95, March 13-18, Los Angeles U.S.A. (1995), hep-th/9507121 [inSPIRE].

[13] A. Strominger, Open p-branes, Phys. Lett. B 383 (1996) 44 [hep-th/9512059] [InSPIRE].

[14] N. Seiberg, Nontrivial fixed points of the renormalization group in six-dimensions, Phys. Lett. B 390 (1997) 169 [hep-th/9609161] [INSPIRE].

[15] E. Witten, Small instantons in string theory, Nucl. Phys. B 460 (1996) 541 [hep-th/9511030] [INSPIRE].

[16] O.J. Ganor and A. Hanany, Small $E_{8}$ instantons and tensionless noncritical strings, Nucl. Phys. B 474 (1996) 122 [hep-th/9602120] [INSPIRE].

[17] D.R. Morrison and C. Vafa, Compactifications of F-theory on Calabi-Yau threefolds. 2., Nucl. Phys. B 476 (1996) 437 [hep-th/9603161] [INSPIRE].

[18] N. Seiberg and E. Witten, Comments on string dynamics in six-dimensions, Nucl. Phys. B 471 (1996) 121 [hep-th/9603003] [INSPIRE].

[19] M. Bershadsky and A. Johansen, Colliding singularities in F-theory and phase transitions, Nucl. Phys. B 489 (1997) 122 [hep-th/9610111] [INSPIRE].

[20] I. Brunner and A. Karch, Branes at orbifolds versus Hanany Witten in six-dimensions, JHEP 03 (1998) 003 [hep-th/9712143] [INSPIRE].

[21] J.D. Blum and K.A. Intriligator, Consistency conditions for branes at orbifold singularities, Nucl. Phys. B 506 (1997) 223 [hep-th/9705030] [INSPIRE].

[22] P.S. Aspinwall and D.R. Morrison, Point-like instantons on K3 orbifolds, Nucl. Phys. B 503 (1997) 533 [hep-th/9705104] [INSPIRE].

[23] K.A. Intriligator, New string theories in six-dimensions via branes at orbifold singularities, Adv. Theor. Math. Phys. 1 (1998) 271 [hep-th/9708117] [INSPIRE].

[24] A. Hanany and A. Zaffaroni, Branes and six-dimensional supersymmetric theories, Nucl. Phys. B 529 (1998) 180 [hep-th/9712145] [INSPIRE].

[25] A. Klemm, W. Lerche, P. Mayr, C. Vafa and N.P. Warner, Selfdual strings and $N=2$ supersymmetric field theory, Nucl. Phys. B 477 (1996) 746 [hep-th/9604034] [INSPIRE].

[26] N.J. Evans, C.V. Johnson and A.D. Shapere, Orientifolds, branes and duality of $4 D$ gauge theories, Nucl. Phys. B 505 (1997) 251 [hep-th/9703210] [INSPIRE].

[27] K. Landsteiner, E. Lopez and D.A. Lowe, $N=2$ supersymmetric gauge theories, branes and orientifolds, Nucl. Phys. B 507 (1997) 197 [hep-th/9705199] [INSPIRE]. 
[28] A. Brandhuber, J. Sonnenschein, S. Theisen and S. Yankielowicz, $M$ theory and Seiberg-Witten curves: orthogonal and symplectic groups, Nucl. Phys. B 504 (1997) 175 [hep-th/9705232] [INSPIRE].

[29] K. Landsteiner and E. Lopez, New curves from branes, Nucl. Phys. B 516 (1998) 273 [hep-th/9708118] [INSPIRE].

[30] A. Kapustin, Solution of $N=2$ gauge theories via compactification to three-dimensions, Nucl. Phys. B 534 (1998) 531 [hep-th/9804069] [INSPIRE].

[31] K. Landsteiner, E. Lopez and D.A. Lowe, Supersymmetric gauge theories from branes and orientifold six planes, JHEP 07 (1998) 011 [hep-th/9805158] [INSPIRE].

[32] P.C. Argyres, R. Maimon and S. Pelland, The M-theory lift of two O6-planes and four D6-branes, JHEP 05 (2002) 008 [hep-th/0204127] [INSPIRE].

[33] L.F. Alday, D. Gaiotto and Y. Tachikawa, Liouville correlation functions from four-dimensional gauge theories, Lett. Math. Phys. 91 (2010) 167 [arXiv:0906.3219] [INSPIRE].

[34] O. Chacaltana and J. Distler, Tinkertoys for Gaiotto duality, JHEP 11 (2010) 099 [arXiv: 1008.5203] [INSPIRE].

[35] O. Chacaltana, J. Distler and Y. Tachikawa, Nilpotent orbits and codimension-two defects of $6 d N=(2,0)$ theories, Int. J. Mod. Phys. A 28 (2013) 1340006 [arXiv:1203.2930] [INSPIRE].

[36] D. Xie, General Argyres-Douglas theory, JHEP 01 (2013) 100 [arXiv: 1204.2270] [INSPIRE].

[37] N. Nekrasov and V. Pestun, Seiberg-Witten geometry of four dimensional $N=2$ quiver gauge theories, arXiv:1211.2240 [INSPIRE].

[38] C. Beem, L. Rastelli and B.C. van Rees, $\mathcal{W}$ symmetry in six dimensions, JHEP 05 (2015) 017 [arXiv: 1404.1079] [INSPIRE].

[39] K. Ohmori, H. Shimizu and Y. Tachikawa, Anomaly polynomial of E-string theories, JHEP 08 (2014) 002 [arXiv: 1404.3887] [INSPIRE].

[40] J.J. Heckman, More on the matter of 6D SCFTs, Phys. Lett. B 747 (2015) 73 [arXiv:1408.0006] [INSPIRE].

[41] K. Ohmori, H. Shimizu, Y. Tachikawa and K. Yonekura, Anomaly polynomial of general $6 d$ SCFTs, PTEP 2014 (2014) 103B07 [arXiv: 1408.5572] [INSPIRE].

[42] K. Intriligator, $6 d, \mathcal{N}=(1,0)$ Coulomb branch anomaly matching, JHEP 10 (2014) 162 [arXiv: 1408.6745] [INSPIRE].

[43] B. Haghighat, A. Klemm, G. Lockhart and C. Vafa, Strings of minimal 6d SCFTs, Fortsch. Phys. 63 (2015) 294 [arXiv:1412.3152] [INSPIRE].

[44] M. Del Zotto, J.J. Heckman, D.S. Park and T. Rudelius, On the defect group of a $6 D$ SCFT, Lett. Math. Phys. 106 (2016) 765 [arXiv:1503.04806] [InSPIRE].

[45] D. Gaiotto and S.S. Razamat, $\mathcal{N}=1$ theories of class $\mathcal{S}_{k}$, JHEP 07 (2015) 073 [arXiv: 1503.05159] [INSPIRE].

[46] K. Ohmori, H. Shimizu, Y. Tachikawa and K. Yonekura, $6 d \mathcal{N}=(1,0)$ theories on $T^{2}$ and class $S$ theories: part I, JHEP 07 (2015) 014 [arXiv:1503.06217] [INSPIRE].

[47] A. Gadde et al., 6d string chains, arXiv:1504.04614 [INSPIRE]. 
[48] S. Franco, H. Hayashi and A. Uranga, Charting class $\mathcal{S}_{k}$ territory, Phys. Rev. D 92 (2015) 045004 [arXiv: 1504.05988] [INSPIRE].

[49] M. Del Zotto, C. Vafa and D. Xie, Geometric engineering, mirror symmetry and $6 d_{(1,0)} \rightarrow 4 d_{(\mathcal{N}=2)}$, JHEP 11 (2015) 123 [arXiv: 1504.08348] [INSPIRE].

[50] A. Hanany and K. Maruyoshi, Chiral theories of class S, JHEP 12 (2015) 080 [arXiv: 1505.05053] [INSPIRE].

[51] C. Beem, M. Lemos, L. Rastelli and B.C. van Rees, The $(2,0)$ superconformal bootstrap, Phys. Rev. D 93 (2016) 025016 [arXiv: 1507.05637] [InSPIRE].

[52] C. Cordova, T.T. Dumitrescu and K. Intriligator, Anomalies, renormalization group flows and the a-theorem in six-dimensional $(1,0)$ theories, JHEP 10 (2016) 080 [arXiv: 1506. 03807] [INSPIRE].

[53] M. Aganagic and N. Haouzi, ADE little string theory on a Riemann surface (and triality), arXiv: 1506.04183 [INSPIRE].

[54] K. Ohmori, H. Shimizu, Y. Tachikawa and K. Yonekura, $6 d \mathcal{N}=(1,0)$ theories on $S^{1} / T^{2}$ and class $S$ theories: part II, JHEP 12 (2015) 131 [arXiv:1508.00915] [INSPIRE].

[55] I. Coman, E. Pomoni, M. Taki and F. Yagi, Spectral curves of $\mathcal{N}=1$ theories of class $\mathcal{S}_{k}$, JHEP 06 (2017) 136 [arXiv: 1512.06079] [INSPIRE].

[56] H. Hayashi, S.-S. Kim, K. Lee, M. Taki and F. Yagi, More on $5 d$ descriptions of $6 d$ SCFTs, JHEP 10 (2016) 126 [arXiv:1512.08239] [INSPIRE].

[57] C. Cordova, T.T. Dumitrescu and K. Intriligator, Deformations of superconformal theories, JHEP 11 (2016) 135 [arXiv:1602.01217] [INSPIRE].

[58] D.R. Morrison and C. Vafa, F-theory and $\mathcal{N}=1$ SCFTs in four dimensions, JHEP 08 (2016) 070 [arXiv: 1604.03560] [inSPIRE].

[59] H.-C. Kim, S. Kim and J. Park, 6d strings from new chiral gauge theories, arXiv: 1608.03919 [INSPIRE].

[60] H. Shimizu and Y. Tachikawa, Anomaly of strings of $6 d \mathcal{N}=(1,0)$ theories, JHEP 11 (2016) 165 [arXiv: 1608.05894] [INSPIRE].

[61] M. Del Zotto and G. Lockhart, On exceptional instanton strings, JHEP 09 (2017) 081 [arXiv: 1609.00310] [INSPIRE].

[62] J.J. Heckman, P. Jefferson, T. Rudelius and C. Vafa, Punctures for theories of class $\mathcal{S}_{\Gamma}$, JHEP 03 (2017) 171 [arXiv: 1609.01281] [INSPIRE].

[63] F. Apruzzi, F. Hassler, J.J. Heckman and I.V. Melnikov, From 6D SCFTs to dynamic GLSMs, Phys. Rev. D 96 (2017) 066015 [arXiv:1610.00718] [INSPIRE].

[64] S.S. Razamat, C. Vafa and G. Zafrir, $4 d \mathcal{N}=1$ from 6d (1,0), JHEP 04 (2017) 064 [arXiv: 1610.09178] [INSPIRE].

[65] C. Cordova, T.T. Dumitrescu and K. Intriligator, Multiplets of superconformal symmetry in diverse dimensions, arXiv:1612.00809 [INSPIRE].

[66] M. Del Zotto, J.J. Heckman and D.R. Morrison, $6 D$ SCFTs and phases of $5 D$ theories, JHEP 09 (2017) 147 [arXiv:1703.02981] [INSPIRE].

[67] B. Haghighat, W. Yan and S.-T. Yau, ADE string chains and mirror symmetry, JHEP 01 (2018) 043 [arXiv: 1705. 05199] [InSPIRE]. 
[68] C.-M. Chang and Y.-H. Lin, Carving out the end of the world or (superconformal bootstrap in six dimensions), JHEP 08 (2017) 128 [arXiv: 1705.05392] [INSPIRE].

[69] N. Mekareeya, K. Ohmori, Y. Tachikawa and G. Zafrir, E $E_{8}$ instantons on type-A ALE spaces and supersymmetric field theories, JHEP 09 (2017) 144 [arXiv:1707.04370] [INSPIRE].

[70] N. Mekareeya, K. Ohmori, H. Shimizu and A. Tomasiello, Small instanton transitions for M5 fractions, JHEP 10 (2017) 055 [arXiv:1707.05785] [INSPIRE].

[71] N. Seiberg, Notes on theories with 16 supercharges, Nucl. Phys. Proc. Suppl. 67 (1998) 158 [hep-th/9705117] [INSPIRE].

[72] O.J. Ganor, D.R. Morrison and N. Seiberg, Branes, Calabi-Yau spaces and toroidal compactification of the $N=1$ six-dimensional $E_{8}$ theory, Nucl. Phys. B 487 (1997) 93 [hep-th/9610251] [INSPIRE].

[73] O.J. Ganor, Compactification of tensionless string theories, hep-th/9607092 [INSPIRE].

[74] M.B. Green, J.H. Schwarz and P.C. West, Anomaly free chiral theories in six-dimensions, Nucl. Phys. B 254 (1985) 327 [InSPIRE].

[75] A. Sagnotti, A note on the Green-Schwarz mechanism in open string theories, Phys. Lett. B 294 (1992) 196 [hep-th/9210127] [inSPIRE].

[76] V. Sadov, Generalized Green-Schwarz mechanism in F-theory, Phys. Lett. B 388 (1996) 45 [hep-th/9606008] [INSPIRE].

[77] D.R. Morrison and W. Taylor, Classifying bases for $6 D$ F-theory models, Central Eur. J. Phys. 10 (2012) 1072 [arXiv:1201.1943] [INSPIRE].

[78] A. Hanany and E. Witten, Type IIB superstrings, BPS monopoles and three-dimensional gauge dynamics, Nucl. Phys. B 492 (1997) 152 [hep-th/9611230] [INSPIRE].

[79] F. Cachazo et al., A geometric unification of dualities, Nucl. Phys. B 628 (2002) 3 [hep-th/0110028] [INSPIRE].

[80] Y. Tachikawa, Six-dimensional D $(N)$ theory and four-dimensional SO-USp quivers, JHEP 07 (2009) 067 [arXiv:0905.4074] [INSPIRE].

[81] N. Drukker, D. Gaiotto and J. Gomis, The virtue of defects in $4 D$ gauge theories and $2 D$ CFTs, JHEP 06 (2011) 025 [arXiv: 1003.1112] [INSPIRE].

[82] Y. Tachikawa, $N=2$ S-duality via outer-automorphism twists, J. Phys. A 44 (2011) 182001 [arXiv: 1009.0339] [INSPIRE].

[83] E. Witten, Toroidal compactification without vector structure, JHEP 02 (1998) 006 [hep-th/9712028] [INSPIRE].

[84] J. de Boer et al., Triples, fluxes and strings, Adv. Theor. Math. Phys. 4 (2002) 995 [hep-th/0103170] [INSPIRE].

[85] Y. Tachikawa, Frozen singularities in M and F-theory, JHEP 06 (2016) 128 [arXiv: 1508.06679] [INSPIRE].

[86] L. Bhardwaj et al., F-theory and the classification of little strings, Phys. Rev. D 93 (2016) 086002 [arXiv: 1511.05565] [INSPIRE].

[87] C. Cordova, T.T. Dumitrescu and X. Yin, Higher derivative terms, toroidal compactification and Weyl anomalies in six-dimensional $(2,0)$ theories, arXiv: 1505.03850 [INSPIRE]. 
[88] J.J. Heckman, D.R. Morrison, T. Rudelius and C. Vafa, Geometry of $6 D$ RG flows, JHEP 09 (2015) 052 [arXiv: 1505.00009] [INSPIRE].

[89] J.J. Heckman, T. Rudelius and A. Tomasiello, $6 D$ RG flows and nilpotent hierarchies, JHEP 07 (2016) 082 [arXiv: 1601.04078] [INSPIRE].

[90] N. Mekareeya, T. Rudelius and A. Tomasiello, T-branes, anomalies and moduli spaces in 6D SCFTs, JHEP 10 (2017) 158 [arXiv:1612.06399] [INSPIRE].

[91] D. Gaiotto, A. Kapustin, N. Seiberg and B. Willett, Generalized global symmetries, JHEP 02 (2015) 172 [arXiv: 1412.5148] [INSPIRE].

[92] M. Del Zotto, J.J. Heckman, D.R. Morrison and D.S. Park, 6D SCFTs and gravity, JHEP 06 (2015) 158 [arXiv: 1412.6526] [InSPIRE].

[93] J.J. Heckman and T. Rudelius, Evidence for C-theorems in 6D SCFTs, JHEP 09 (2015) 218 [arXiv: 1506. 06753] [INSPIRE].

[94] V. Kumar, D.R. Morrison and W. Taylor, Global aspects of the space of $6 D N=1$ supergravities, JHEP 11 (2010) 118 [arXiv:1008.1062] [INSPIRE].

[95] I.R. Klebanov and M.J. Strassler, Supergravity and a confining gauge theory: Duality cascades and chi SB resolution of naked singularities, JHEP 08 (2000) 052 [hep-th/0007191] [INSPIRE].

[96] N. Seiberg, Electric-magnetic duality in supersymmetric non-Abelian gauge theories, Nucl. Phys. B 435 (1995) 129 [hep-th/9411149] [INSPIRE].

[97] S. Schäfer-Nameki and T. Weigand, F-theory and 2d (0,2) theories, JHEP 05 (2016) 059 [arXiv: 1601.02015] [INSPIRE].

[98] F. Apruzzi, F. Hassler, J.J. Heckman and I.V. Melnikov, UV completions for non-critical strings, JHEP 07 (2016) 045 [arXiv: 1602.04221] [INSPIRE].

[99] A. Gadde, S. Gukov and P. Putrov, (0,2) trialities, JHEP 03 (2014) 076 [arXiv: 1310.0818 ] [INSPIRE].

[100] S. Franco, S. Lee and R.-K. Seong, Brane brick models and $2 d(0,2)$ triality, JHEP 05 (2016) 020 [arXiv: 1602.01834] [InSPIRE].

[101] S. Franco, S. Lee, R.-K. Seong and C. Vafa, Brane brick models in the mirror, JHEP 02 (2017) 106 [arXiv: 1609.01723] [InSPIRE].

[102] S. Hohenegger, A. Iqbal and S.-J. Rey, Instanton-monopole correspondence from M-branes on $\mathbb{S}^{1}$ and little string theory, Phys. Rev. D 93 (2016) 066016 [arXiv:1511.02787] [INSPIRE].

[103] A. Hanany and A. Zaffaroni, Issues on orientifolds: On the brane construction of gauge theories with $\mathrm{SO}(2 N)$ global symmetry, JHEP 07 (1999) 009 [hep-th/9903242] [INSPIRE].

[104] O. Bergman and D. Rodriguez-Gomez, 5d quivers and their AdS 6 duals, JHEP 07 (2012) 171 [arXiv:1206.3503] [INSPIRE].

[105] I. Bah, A. Hanany, K. Maruyoshi, S.S. Razamat, Y. Tachikawa and G. Zafrir, $4 d \mathcal{N}=1$ from $6 d \mathcal{N}=(1,0)$ on a torus with fluxes, JHEP 06 (2017) 022 [arXiv:1702.04740] [INSPIRE].

[106] M.R. Douglas and G.W. Moore, D-branes, quivers and ALE instantons, hep-th/9603167 [INSPIRE]. 
[107] C.V. Johnson and R.C. Myers, Aspects of type IIB theory on ALE spaces, Phys. Rev. D 55 (1997) 6382 [hep-th/9610140] [INSPIRE].

[108] A.E. Lawrence, N. Nekrasov and C. Vafa, On conformal field theories in four-dimensions, Nucl. Phys. B 533 (1998) 199 [hep-th/9803015] [INSPIRE].

[109] O. Aharony and Y. Tachikawa, A holographic computation of the central charges of $D=4$, $N=2$ SCFTs, JHEP 01 (2008) 037 [arXiv:0711.4532] [INSPIRE].

[110] Y. Hyakutake, Y. Imamura and S. Sugimoto, Orientifold planes, type-I Wilson lines and non-BPS D-branes, JHEP 08 (2000) 043 [hep-th/0007012] [INSPIRE].

[111] A. Hanany and B. Kol, On orientifolds, discrete torsion, branes and M-theory, JHEP 06 (2000) 013 [hep-th/0003025] [INSPIRE].

[112] O. Bergman, E.G. Gimon and S. Sugimoto, Orientifolds, RR torsion and k-theory, JHEP 05 (2001) 047 [hep-th/0103183] [INSPIRE].

[113] D.R. Morrison and N. Seiberg, Extremal transitions and five-dimensional supersymmetric field theories, Nucl. Phys. B 483 (1997) 229 [hep-th/9609070] [INSPIRE].

[114] J.A. Minahan and D. Nemeschansky, Superconformal fixed points with E(n) global symmetry, Nucl. Phys. B 489 (1997) 24 [hep-th/9610076] [INSPIRE].

[115] H. Hayashi and K. Ohmori, 5d/6d DE instantons from trivalent gluing of web diagrams, JHEP 06 (2017) 078 [arXiv: 1702.07263] [INSPIRE].

[116] O. Chacaltana, J. Distler and Y. Tachikawa, Gaiotto duality for the twisted $A_{2 N-1}$ series, JHEP 05 (2015) 075 [arXiv: 1212.3952] [INSPIRE].

[117] M. Bershadsky and C. Vafa, Global anomalies and geometric engineering of critical theories in six-dimensions, hep-th/9703167 [INSPIRE]. 